\title{
Two Palladium-Catalyzed Domino Reactions from One Set of Substrates/ Reagents: Efficient Synthesis of Substituted Indenes and cis-Stilbenoid Hydrocarbons from the Same Internal Alkynes and Hindered Grignard Reagents
}

\author{
Cheng-Guo Dong, Pik Yeung and Qiao-Sheng Hu* \\ Department of Chemistry, College of Staten Island and the Graduate Center of the City \\ University of New York, Staten Island, New York 10314
}

Supporting Information

General: NMR spectra were recorded on Varian $200 \mathrm{MHz}$ or $600 \mathrm{MHz}$ spectrometers. Chemical shifts were reported in ppm down field from internal tetramethylsilane. All yields reported refer to isolated yields (average of two runs) unless otherwise indicated, and the product purity was estimated to be greater than $95 \%$ as determined by ${ }^{1} \mathrm{H}$ NMR. Melting points were measured on a Fisher-Johns Melting Point Apparatus and uncorrected. Elemental analyses were performed by Atlantic Microlab, Inc. THF was distilled from sodium/benzophenone ketyl. 2,6Dimethylphenylmagnesium bromide, 2-mesitylmagnesium bromide, 1-phenyl-1-propyne, 3hexyne, 1,2-dibromethane, anhydrous iron(III) chloride were purchased from Aldrich and used directly. $\mathrm{Pd}(\mathrm{OAc})_{2}$ was a gift from Frontier Scientific, Inc. $\mathrm{PPh}_{3}$ was purchased from Acros Organics and used directly. Anhydrous copper(II) chloride, Anhydrous copper(II) sulfate, silver carbonate, and Iron(III) chloride were purchased from Stem Chemical Inc. and were used as received. Other chemical reagents were purchased from Alfa Aesar and used without further purification. Bromopentamethylbenzene, pentamethylphenylmagnesium bromide were prepared according to reported methods. ${ }^{1,2}$ 2-Bromo-1-ethyl-3-methylbenzene, 2-bromo-1,3diethylbenzene and 2-bromo-1-isopropyl-3-methylbenzene were prepared according to literature procedures. $^{3} \quad$ 1,2-Bis(4-methoxyphenyl)acetylene and 4-(1-Hexyn-1-yl)methylbenzene was prepared according to reported method. ${ }^{4}$

\section{General Procedures for $\mathrm{Pd}(\mathrm{OAc})_{2}$-Promoted Domino Reaction of Diphenylacetylene with Mesitylmagnesium Bromide:}

A. In glove box with nitrogen atmosphere, to a mixture of diphenylacetylene $(44.5 \mathrm{mg}, 0.25$ $\mathrm{mmol}$ ) and $0.5 \mathrm{ml}$ THF (in a schlenk flask) was added palladium acetate (56 $\mathrm{mg}, 0.25$ mmol). After stirred for 5-10 minutes, Grignard reagent $(0.65 \mathrm{~mL}, 1 \mathrm{M}$ in THF, 0.65 mmol) was added. The mixture was allowed to stir at room temperature or $60^{\circ} \mathrm{C}$ (oil bath) or refluxing for 20 hours. After quenched with water, the reaction mixture was extracted with ethyl acetate $(15 \mathrm{~mL} \times 3)$. The organic layer was washed with brine and the solvent was evaporated under vacuum. The reaction mixtures were analyzed by ${ }^{1} \mathrm{H} \mathrm{NMR}$, from which the reaction conversion and the ratios of cyclization product : cross-coupling product : self-coupling product were be obtained.

B. In glove box with nitrogen atmosphere, to a mixture of diphenylacetylene ( $44.5 \mathrm{mg}, 0.25$ $\mathrm{mmol}$ ) and $0.5 \mathrm{~mL}$ THF (in a schlenk flask) was added palladium acetate (56 $\mathrm{mg}, 0.25$ 
mmol) and $\mathrm{PPh}_{3}$ (2 equiv., $131 \mathrm{mg}, 0.5 \mathrm{mmol}$, or 4 equiv., $262 \mathrm{mg}, 1.0 \mathrm{mmol}$ ). After stirred for 5-10 minutes, Grignard reagent (1.0 mL, 1M in THF, $1.0 \mathrm{mmol})$ was added. The mixture was allowed to stir under room temperature or $60^{\circ} \mathrm{C}$ (oil bath) or refluxing for 20 hours. After quenched with water, the reaction mixture was extracted with ethyl acetate $(15 \mathrm{~mL} \times 3)$. The organic layer was washed with brine and the solvent was evaporated under vacuum. The reaction mixtures were analyzed by ${ }^{1} \mathrm{H}$ NMR, from which the reaction conversion and the ratios of cyclization product: cross-coupling product : self-coupling product were be obtained.

\section{General Procedure of the Oxidant Screening for $\mathrm{Pd}(\mathrm{OAc})_{2}$-Catalyzed Domino Reaction of Diphenylacetylene with Mesitylmagnesium Bromide:}

In glove box with nitrogen atmosphere, to a mixture of diphenylacetylene $(89 \mathrm{mg}, 0.5$ mmol), oxidant (0.5 mmol) and $0.5 \mathrm{~mL}$ THF (in a Schlenk flask) was added palladium acetate (3.4 mg, $0.015 \mathrm{mmol}$ ). After stirred for 5-10 minutes, Grignard reagent $(1.25 \mathrm{~mL}, 1 \mathrm{M}$ in THF, $1.25 \mathrm{mmol}$ ) was added. The mixture was allowed to stir under $60^{\circ} \mathrm{C}$ (oil bath) for 20 hours. After quenched with water, the reaction mixture was extracted with ethyl acetate $(15 \mathrm{~mL} \times 3)$. The organic layer was washed with brine and the solvent was evaporated under vacuum. The crude reaction mixtures were analyzed by ${ }^{1} \mathrm{H}$ NMR. Flash chromatography on silica gel (hexane: ethyl acetate $=100: 0$ to $90: 10)$ gave the cyclization products.

\section{General Procedure for $\mathrm{Pd}(\mathrm{OAc})_{2}$-Catalyzed Annulative Domino Reaction of Internal Alkynes with Hindered Grignard Reagents:}

In glove box with nitrogen atmosphere, to a mixture of alkyne $(0.5 \mathrm{mmol})$, 1,2dibromoethane $(0.75 \mathrm{mmol}, 65 \mu \mathrm{l})$ and $0.5 \mathrm{~mL}$ THF (in a schlenk flask) was added palladium acetate $(3.4 \mathrm{mg}, 0.015 \mathrm{mmol})$. After stirred for 5-10 minutes, Grignard reagent $(1.25 \mathrm{~mL}, 1 \mathrm{M}$ in THF, $1.25 \mathrm{mmol}$ ) was added. The mixture was allowed to stir under $60^{\circ} \mathrm{C}$ (oil bath) for 20 hours. After quenched with water, the reaction mixture was extracted with ethyl acetate $(15 \mathrm{~mL} \times 3)$. The organic layer was washed with brine and the solvent was evaporated under vacuum. Flash chromatography on silica gel (hexane: ethyl acetate $=100: 0$ to $90: 10$ ) gave the cyclization products.

4,6-Dimethyl-2,3-diphenyl-1H-indene (1): white solid. m. p.: 86-87 ${ }^{\circ} \mathrm{C} .{ }^{1} \mathrm{H} \mathrm{NMR}\left(\mathrm{CDCl}_{3}, 600\right.$ MHz): $\delta 7.403 \sim 7.345(\mathrm{~m}, 3 \mathrm{H}), 7.313(\mathrm{~d}, \mathrm{~J}=7.2 \mathrm{~Hz}, 2 \mathrm{H}), 7.209(\mathrm{~s}, 1 \mathrm{H}), 7.178(\mathrm{~d}, \mathrm{~J}=8.4 \mathrm{~Hz}, 2 \mathrm{H})$, $7.139(\mathrm{t}, \mathrm{J}=7.2 \mathrm{~Hz}, 2 \mathrm{H}), 7.097(\mathrm{t}, \mathrm{J}=7.2 \mathrm{~Hz}, 1 \mathrm{H}), 6.819(\mathrm{~s}, 1 \mathrm{H}), 3.861(\mathrm{~s}, 2 \mathrm{H}), 2.368(\mathrm{~s}, 3 \mathrm{H})$, $1.808(\mathrm{~s}, 3 \mathrm{H}) .{ }^{13} \mathrm{C} \mathrm{NMR}\left(\mathrm{CDCl}_{3}, 150 \mathrm{MHz}\right): \delta 143.118,141.612,141.367,140.054,139.278$, 136.729, 134.756, 131.576, 130.386, 129.620, 128.560, 127.998, 127.851, 127.159, 126.457, 122.188, 40.595, 21.168, 19.834. Anal. calcd. for $\mathrm{C}_{23} \mathrm{H}_{20}$ : C, 93.20\%; H, 6.80\%. Found: C, $93.00 \% ; \mathrm{H}, 6.69 \%$.

4-Methyl-2,3-diphenyl-1H-indene (2): light yellow solid. M. p.: $118-119^{\circ} \mathrm{C} .{ }^{1} \mathrm{H}$ NMR $\left(\mathrm{CDCl}_{3}, 600 \mathrm{MHz}\right): \delta 7.412 \sim 7.367(\mathrm{~m}, 4 \mathrm{H}), 7.325(\mathrm{~d}, \mathrm{~J}=7.8 \mathrm{~Hz}, 2 \mathrm{H}), 7.194(\mathrm{~d}, \mathrm{~J}=7.8 \mathrm{~Hz}, 2 \mathrm{H})$, 7.160 (d, J= 7.8 Hz, 2H), 7.127 (t, J=7.8 Hz, 2H), 6.992 (d, J=7.8 Hz, 1H), 3.905 (s, 2H), 1.848 $(\mathrm{s}, 3 \mathrm{H}) .{ }^{13} \mathrm{C} \mathrm{NMR}\left(\mathrm{CDCl}_{3}, 150 \mathrm{MHz}\right): \delta 144.101,142.721,141.478,141.152,139.137,136.584$, 131.940, 129.669, 129.553, 128.563, 128.022, 127.969, 127.225, 126.639, 124.933, 121.334, 40.844, 20.012. Anal. calcd. for $\mathrm{C}_{22} \mathrm{H}_{18}$ : C, 93.57\%; H, 6.43\%. Found: C, 93.35\%; H, 6.47\%. 
4,5,6,7-Tetramethyl-2,3-diphenyl-1H-indene (3): off-white solid. M. p.: 139-141 ${ }^{\circ} \mathrm{C} .{ }^{1} \mathrm{H}$ NMR $\left(\mathrm{CDCl}_{3}, 600 \mathrm{MHz}\right): \delta 7.383(\mathrm{t}, \mathrm{J}=7.2 \mathrm{~Hz}, 2 \mathrm{H}), 7.345(\mathrm{t}, \mathrm{J}=7.2 \mathrm{~Hz}, 1 \mathrm{H}), 7.292(\mathrm{~d}, \mathrm{~J}=7.2 \mathrm{~Hz}, 1 \mathrm{H})$, 7.160 (d, J=7.8 Hz, 2H), 7.134 (t, J=7.8 Hz, 2H), 7.090 (t, J=7.8 Hz, 1H), 3.795 (s, 2H), 2.377 (s, 3H), 2.299 (s, 3H), 2.194 (s, 3H), 1.795 (s, 3H). ${ }^{13} \mathrm{C} \mathrm{NMR}\left(\mathrm{CDCl}_{3}, 150 \mathrm{MHz}\right): \delta 142.156$, $141.415,140.474,139.912$, 139.147, 137.132, 134.573, 132.119, 129.676, 128.636, 128.205, 128.026, 127.906, 127.011, 126.319, 40.573, 16.368, 16.227, 16.119, 16.048. Anal. calcd. for $\mathrm{C}_{25} \mathrm{H}_{24}: \mathrm{C}, 92.54 \%$; H, 7.46\%. Found: C, 92.33\%; H, 7.45\%.

6-Methoxy-4-methyl-2,3-diphenyl-1H-indene (4): white solid. M. p.: $108-109^{\circ} \mathrm{C} .{ }^{1} \mathrm{H}$ NMR $\left(\mathrm{CDCl}_{3}, 600 \mathrm{MHz}\right): \delta 7.387(\mathrm{t}, \mathrm{J}=7.8 \mathrm{~Hz}, 2 \mathrm{H}), 7.361(\mathrm{t}, \mathrm{J}=7.2 \mathrm{~Hz}, 1 \mathrm{H}), 7.317(\mathrm{~d}, \mathrm{~J}=7.8 \mathrm{~Hz}, 2 \mathrm{H})$, $7.167 \sim 7.121(\mathrm{~m}, 4 \mathrm{H}), 7.087(\mathrm{t}, \mathrm{J}=7.2 \mathrm{~Hz}, 1 \mathrm{H}), 6.980(\mathrm{~d}, \mathrm{~J}=1.8 \mathrm{~Hz}, 1 \mathrm{H}), 6.560(\mathrm{~d}, \mathrm{~J}=1.8 \mathrm{~Hz}$, $1 \mathrm{H}), 3.867(\mathrm{~s}, 2 \mathrm{H}), 3.836(\mathrm{~s}, 3 \mathrm{H}), 1.813(\mathrm{~s}, 3 \mathrm{H}) .{ }^{13} \mathrm{C} \mathrm{NMR}\left(\mathrm{CDCl}_{3}, 150 \mathrm{MHz}\right): \delta 157.782$, $144.656,141.132,139.239,138.843,137.561,136.743,132.815,129.592,128.585,127.995$, 127.686, 127.177, 126.275, 115.188, 107.412, 55.463, 40.813, 20.069. Anal. calcd. for $\mathrm{C}_{23} \mathrm{H}_{20} \mathrm{O}$ : C, $88.43 \%$; H, 6.45\%. Found: C, 88.34\%; H, 6.34\%.

2,3-Bis(4-methoxyphenyl)-4,6-dimethyl-1H-indene (5): yellow solid. M. p.: 116-118 ${ }^{\circ} \mathrm{C} .{ }^{1} \mathrm{H}$ NMR $\left(\mathrm{CDCl}_{3}, 600 \mathrm{MHz}\right): \delta 7.211(\mathrm{~d}, \mathrm{~J}=9.0 \mathrm{~Hz}, 2 \mathrm{H}), 7.179(\mathrm{~s}, 1 \mathrm{H}), 7.139(\mathrm{~d}, \mathrm{~J}=9.0 \mathrm{~Hz}, 2 \mathrm{H})$, 6.948 (d, J=8.4 Hz, 2H), 6.802 (s, 1H), 6.703 (d, J=8.4 Hz, 2H), 3.868 (s, 3H), 3.807 (s, 2H), $3.745(\mathrm{~s}, 3 \mathrm{H}), 2.359(\mathrm{~s}, 3 \mathrm{H}), 1.831(\mathrm{~s}, 3 \mathrm{H}) .{ }^{13} \mathrm{C} \mathrm{NMR}\left(\mathrm{CDCl}_{3}, 150 \mathrm{MHz}\right): \delta 158.670,158.066$, $142.778,142.009$, 139.741, 139.422, 134.233, 131.572, 131.182, 130.691, 130.301, 129.497, 128.890, 122.076, 114.012, 113.419, 55.115, 55.068, 40.419, 21.115, 19.875. Anal. calcd. for $\mathrm{C}_{25} \mathrm{H}_{24} \mathrm{O}_{2}$ : C, 84.24\%; H, 6.79\%. Found: C, 83.89\%; H, 6.81\%.

2,3-bis(4-methoxyphenyl)-4-methyl-1H-indene (6): white solid. M. p.: $131-132{ }^{\circ} \mathrm{C} .{ }^{1} \mathrm{H}$ NMR $\left(\mathrm{CDCl}_{3}, 600 \mathrm{MHz}\right): \delta 7.354(\mathrm{~d}, \mathrm{~J}=7.8 \mathrm{~Hz}, 1 \mathrm{H}), 7.221(\mathrm{~d}, \mathrm{~J}=9.0 \mathrm{~Hz}, 2 \mathrm{H}), 7.152$ (d, J= $8.4 \mathrm{~Hz}$, 2H), 7.089 (t, J=7.8 Hz, 1H), $6.971(\mathrm{~d}, \mathrm{~J}=7.2 \mathrm{~Hz}, 1 \mathrm{H}), 6.952(\mathrm{~d}, \mathrm{~J}=9.0 \mathrm{~Hz}, 2 \mathrm{H}), 6.710(\mathrm{~d}, \mathrm{~J}=8.4$ $\mathrm{Hz}, 2 \mathrm{H}), 3.871$ (s, 3H), 3.845 (s, 2H), 3.749 (s, 3H), 1.868 (s, 3H). ${ }^{13} \mathrm{C} \mathrm{NMR}\left(\mathrm{CDCl}_{3}, 150\right.$ $\mathrm{MHz}): \delta 158.742,158.226,144.545,142.407,140.859,139.577,131.580,131.457,130.766$, 129.499, 129.362, 129.049, 124.506, 121.210, 114.041, 113.466, 55.162, 55.113, 40.667, 20.056. Anal. calcd. for $\mathrm{C}_{24} \mathrm{H}_{22} \mathrm{O}_{2}$ : C, 84.18\%; H, 6.48\%. Found: $\mathrm{C}, 83.84 \% ; \mathrm{H}, 6.38 \%$.

2,3-Diethyl-4,6-dimethyl-1H-indene (7): colorless liquid. ${ }^{1} \mathrm{H} \mathrm{NMR}\left(\mathrm{CDCl}_{3}, 200 \mathrm{MHz}\right): \delta 7.056$ $(\mathrm{s}, 1 \mathrm{H}), 6.825(\mathrm{~s}, 1 \mathrm{H}), 3.217(\mathrm{~s}, 2 \mathrm{H}), 2.640(\mathrm{q}, \mathrm{J}=7.6 \mathrm{~Hz}, 2 \mathrm{H}), 2.537$ (s, 3H), 2.438 (q, J= 7.6 Hz, 2H), $2.320(\mathrm{~s}, 3 \mathrm{H}), 1.130(\mathrm{t}, \mathrm{J}=7.6 \mathrm{~Hz}, 3 \mathrm{H}), 1.127(\mathrm{t}, \mathrm{J}=7.6 \mathrm{~Hz}, 3 \mathrm{H}) .{ }^{13} \mathrm{C} \mathrm{NMR}\left(\mathrm{CDCl}_{3}, 150\right.$ $\mathrm{MHz}): \delta 143.869,143.233,141.288,139.182,132.951,129.939,129.173,122.138,39.376$, 21.261, 20.970, 19.889, 19.590, 15.290, 14.644 .

2,3-Diethyl-4-methyl-1H-indene (8): colorless liquid. ${ }^{1} \mathrm{H} \mathrm{NMR}\left(\mathrm{CDCl}_{3}, 200 \mathrm{MHz}\right): \delta 7.230(\mathrm{t}$, $\mathrm{J}=3.6 \mathrm{~Hz}, 1 \mathrm{H}), 7.004(\mathrm{~d}, \mathrm{~J}=3.6 \mathrm{~Hz}, 1 \mathrm{H}), 7.699(\mathrm{~d}, \mathrm{~J}=3.6 \mathrm{~Hz}, 1 \mathrm{H}), 3.256(\mathrm{~s}, 2 \mathrm{H}), 2.667(\mathrm{q}, \mathrm{J}=$ $7.2 \mathrm{~Hz}, 2 \mathrm{H}), 2.582(\mathrm{~s}, 3 \mathrm{H}), 2.462(\mathrm{q}, \mathrm{J}=7.2 \mathrm{~Hz}, 2 \mathrm{H}), 1.144(\mathrm{t}, \mathrm{J}=7.2 \mathrm{~Hz}, 6 \mathrm{H}) .{ }^{13} \mathrm{C} \mathrm{NMR}\left(\mathrm{CDCl}_{3}\right.$, $150 \mathrm{MHz}): \delta 144.397,143.993,143.470,139.447,129.575,129.175,123.400,121.185,39.575$, 21.298, 19.901, 19.750, 15.320, 14.625 .

3-Butyl-4,6-dimethyl-2-p-tolyl-1H-indene/2-butyl-4,6-dimethyl-3-p-tolyl-1H-indene (9): ${ }^{1} \mathrm{H}$ NMR showed a 91: 9 ratio. Analytic sample of 3-butyl-4,6-dimethyl-2-p-tolyl-1H-indene was obtained by recrystalization of the mixture of 3-butyl-4,6-dimethyl-2- $p$-tolyl-1H-indene and 2butyl-4,6-dimethyl-3- $p$-tolyl-1H-indene in hexanes. white solid. M. p.: $81-82{ }^{\circ} \mathrm{C}$. ${ }^{1} \mathrm{H}$ NMR $\left(\mathrm{CDCl}_{3}, 600 \mathrm{MHz}\right): \delta 7.276(\mathrm{~d}, \mathrm{~J}=7.8 \mathrm{~Hz}, 2 \mathrm{H}), 7.204(\mathrm{~d}, \mathrm{~J}=7.8 \mathrm{~Hz}, 2 \mathrm{H}), 7.127(\mathrm{~s}, 1 \mathrm{H}), 6.890(\mathrm{~s}$, 1H), 3.600 (s, 2H), $2.733(\mathrm{t}, \mathrm{J}=7.8 \mathrm{~Hz}, 2 \mathrm{H}), 2.583(\mathrm{~s}, 3 \mathrm{H}), 2.382(\mathrm{~s}, 3 \mathrm{H}), 2.352$ (s, 3H), 1.604 
$(\mathrm{m}, 2 \mathrm{H}), 1.375(\mathrm{~m}, 2 \mathrm{H}), 0.894(\mathrm{t}, \mathrm{J}=7.2 \mathrm{~Hz}, 3 \mathrm{H}) .{ }^{13} \mathrm{C} \mathrm{NMR}\left(\mathrm{CDCl}_{3}, 150 \mathrm{MHz}\right): \delta 144.160$, $141.320,140.432$, 140.397, 136.163, 135.401, 133.986, 130.434, 130.300, 128.973, 128.267, $122.222,41.700,33.074,27.482,22.795,21.166,21.051,19.720,13.889$. The structure of $3-$ butyl-4,6-dimethyl-2-p-tolyl-1H-indene was established by NOE effect: NOE effect observed when irradiated at the peak at $\delta 3.600$ ppm: $7.276(\mathrm{~d}, 0.60 \%), 7.127$ (s, 0.65\%). Anal. calcd. for $\mathrm{C}_{22} \mathrm{H}_{26}$ : C, 90.98\%; H, 9.02\%. Found: C, 90.74\%; H, 9.05\%.

3,4,6-Trimethyl-2-phenyl-1H-indene (10): ${ }^{1} \mathrm{H}$ NMR showed a 92: 8 ratio. Analytic sample of 3,4,6-trimethyl-2-phenyl-1H-indene was obtained by recrystalization of the mixture of 3,4,6trimethyl-2-phenyl-1H-indene and 2,4,6-trimethyl-3-phenyl-1H-indene in hexanes. White solid. m.p.: $108-110^{\circ} \mathrm{C} .{ }^{1} \mathrm{H}$ NMR $\left(\mathrm{CDCl}_{3}, 600 \mathrm{MHz}\right) \delta 7.414 \sim 7.389(\mathrm{~m}, 4 \mathrm{H}), 7.271(\mathrm{t}, \mathrm{J}=6.6 \mathrm{~Hz}$, $1 \mathrm{H}), 7.130(\mathrm{~s}, 1 \mathrm{H}), 6.881(\mathrm{~s}, 1 \mathrm{H}), 3.633(\mathrm{~s}, 3 \mathrm{H}), 2.618(\mathrm{~s}, 3 \mathrm{H}), 2.419(\mathrm{~s}, 3 \mathrm{H}), 2.357(\mathrm{~s}, 3 \mathrm{H}) .{ }^{13} \mathrm{C}$ NMR $\left(\mathrm{CDCl}_{3}, 150 \mathrm{MHz}\right) \delta 143.6,142.0,140.2,138.0,136.0,134.3,130.9,130.2,128.6,128.2$, $126.4,122.2,41.2,21.1,20.3,15.6$. The structure of 3,4,6-trimethyl-2-phenyl-1H-indene was established by NOE effect: NOE effect observed when irradiated at the peak at $\delta 3.633$ ppm: $7.400 \sim 7.390$ (m, 0.9\%), 7.130 (s, 0.65\%).

3,4-Dimethyl-2-phenyl-1H-indene (11): ${ }^{1} \mathrm{H}$ NMR showed a 90: 10 ratio. Analytic sample of 3,4-dimethyl-2-phenyl-1H-indene was obtained by recrystallization of the mixture of 3,4dimethyl-2-phenyl-1H-indene and 2,4-dimethyl-3-phenyl-1H-indene in hexanes. White solid. m.p.: $75-77^{\circ} \mathrm{C} .{ }^{1} \mathrm{H} \mathrm{NMR}\left(\mathrm{CDCl}_{3}, 600 \mathrm{MHz}\right) \delta 7.426 \sim 7.390(\mathrm{~m}, 4 \mathrm{H}), 7.311(\mathrm{~d}, \mathrm{~J}=7.2 \mathrm{~Hz}, 1 \mathrm{H})$, $7.282(\mathrm{~d}, \mathrm{~J}=7.2 \mathrm{~Hz}, 1 \mathrm{H}), 7.093(\mathrm{t}, \mathrm{J}=7.2 \mathrm{~Hz}, 1 \mathrm{H}), 7.058(\mathrm{~d}, \mathrm{~J}=7.2 \mathrm{~Hz}, 1 \mathrm{H}), 3.674(\mathrm{~s}, 2 \mathrm{H})$, $2.665(\mathrm{~s}, 3 \mathrm{H}), 2.440(\mathrm{~s}, 3 \mathrm{H}) .{ }^{13} \mathrm{C} \mathrm{NMR}\left(\mathrm{CDCl}_{3}, 150 \mathrm{MHz}\right) \delta 144.6,143.3,141.3,137.9,136.2$, $131.3,129.4,128.6,128.3,126.6,124.6,121.4,41.4,20.4,15.6$. The structure of 3,4-dimethyl-2phenyl-1H-indene was established by NOE effect: NOE effect observed when irradiated at the peak at $\delta 3.674$ ppm: $7.421 \sim 7.410(\mathrm{~m}, 0.8 \%), 7.311(\mathrm{~d}, 0.6 \%)$.

3-Ethyl-4-methyl-2-phenyl-1H-indene (12): ${ }^{1} \mathrm{H}$ NMR showed a 89: 11 ratio. Analytic sample of 3-ethyl-4-methyl-2-phenyl-1H-indene was obtained by recrystallization of the mixture of 3ethyl-4-methyl-2-phenyl-1H-indene and 2-ethyl-4-methyl-3-phenyl-1H-indene in hexanes. White solid. m. p.: 46-47 ${ }^{\circ} \mathrm{C} .{ }^{1} \mathrm{H}$ NMR $\left(\mathrm{CDCl}_{3}, 600 \mathrm{MHz}\right) \delta 7.405 \sim 7.397(\mathrm{~m}, 4 \mathrm{H}), 7.316(\mathrm{~d}, \mathrm{~J}=6.6 \mathrm{~Hz}$, 1H), 7.297 (m, 1H), 7.103 (t, J = 7.2 Hz, 1H), 7.074 (d, J = 7.2 Hz, 1H), 3.659 (s, 2H), 2.789 (q, $\mathrm{J}=7.2 \mathrm{~Hz}, 2 \mathrm{H}), 2.654(\mathrm{~s}, 3 \mathrm{H}), 1.269(\mathrm{t}, \mathrm{J}=7.2 \mathrm{~Hz}, 3 \mathrm{H}) .{ }^{13} \mathrm{C} \mathrm{NMR}\left(\mathrm{CDCl}_{3}, 150 \mathrm{MHz}\right) \delta 143.8$, 143.6, 142.4, 141.2, 138.1, 130.8, 129.6, 128.4, 128.3, 126.7, 124.5, 121.4, 41.9, 20.6, 19.8, 15.6.

3,4-Diethyl-2-phenyl-1H-indene (13): ${ }^{1} \mathrm{H}$ NMR showed a 85: 15 ratio. Analytic sample of 3,4diethyl-2-phenyl-1H-indene was obtained by recrystallization of the mixture of 3,4-diethyl-2phenyl-1H-indene and 2,4-diethyl-3-phenyl-1H-indene in hexanes. White solid. m. p.: 65-66 ${ }^{\circ} \mathrm{C}$. ${ }^{1} \mathrm{H} \mathrm{NMR}\left(\mathrm{CDCl}_{3}, 600 \mathrm{MHz}\right) \delta 7.425 \sim 7.389(\mathrm{~m}, 4 \mathrm{H}), 7.323(\mathrm{~d}, \mathrm{~J}=7.8 \mathrm{~Hz}, 1 \mathrm{H}), 7.307 \sim 7.292$ $(\mathrm{m}, 1 \mathrm{H}), 7.161(\mathrm{t}, \mathrm{J}=7.8 \mathrm{~Hz}, 1 \mathrm{H}), 7.144(\mathrm{~d}, \mathrm{~J}=7.2 \mathrm{~Hz}, 1 \mathrm{H}), 3.661(\mathrm{~s}, 2 \mathrm{H}), 2.977$ (q, J = 7.8 Hz, 2H), 2.768 (q, J = 7.2 Hz, 2H), 1.300 (t, J = 7.2 Hz, 3H), 1.261( t, J = 7.8 Hz, 3H). ${ }^{13} \mathrm{C} \mathrm{NMR}$ $\left(\mathrm{CDCl}_{3}, 150 \mathrm{MHz}\right) \delta 144.0,142.4,142.1,141.6,138.2,137.7,128.4,128.3,127.8,126.7,124.7$, 121.3, 42.0, 25.5, 20.6, 17.1, 15.6. The structure of 3,4-diethyl-2-phenyl-1H-indene was established by NOE effect: NOE effect observed when irradiated at the peak at $\delta 3.661 \mathrm{ppm}$ : $7.412 \sim 7.400(\mathrm{~m}, 0.6 \%), 7.323(\mathrm{~d}, 0.3 \%)$. 
4-Ethyl-2,3-diphenyl-1H-indene (14): Off-white solid. m. p.: 80-81 ${ }^{\circ} \mathrm{C} .{ }^{1} \mathrm{H}$ NMR $\left(\mathrm{CDCl}_{3}, 600\right.$ MHz) $\delta 7.403 \sim 7.365(\mathrm{~m}, 4 \mathrm{H}), 7.344(\mathrm{~d}, \mathrm{~J}=7.8 \mathrm{~Hz}, 1 \mathrm{H}), 7.341(\mathrm{~d}, \mathrm{~J}=7.8 \mathrm{~Hz}, 1 \mathrm{H}), 7.181(\mathrm{t}, \mathrm{J}=$ $7.2 \mathrm{~Hz}, 2 \mathrm{H}), 7.156 \sim 7.140(\mathrm{~m}, 3 \mathrm{H}), 7.130 \sim 7.108(\mathrm{~m}, 1 \mathrm{H}), 7.069(\mathrm{~d}, \mathrm{~J}=7.8 \mathrm{~Hz}, 1 \mathrm{H}), 3.902(\mathrm{~s}$, 2H), $2.216(\mathrm{q}, \mathrm{J}=7.2 \mathrm{~Hz}, 2 \mathrm{H}), 0.872(\mathrm{t}, \mathrm{J}=7.2 \mathrm{~Hz}, 3 \mathrm{H}).) .{ }^{13} \mathrm{C} \mathrm{NMR}\left(\mathrm{CDCl}_{3}, 150 \mathrm{MHz}\right) \delta$ $143.4,143.0,141.5,141.3,139.2,138.6,136.6,129.5,128.5,128.1,128.0,127.9,127.3,126.6$, $125.1,121.2,41.0,25.0,16.3$.

4-Isopropyl-2,3-diphenyl-1H-indene (15): White solid. m. p.: 79-80 ${ }^{\circ} \mathrm{C} .{ }^{1} \mathrm{H} \mathrm{NMR}\left(\mathrm{CDCl}_{3}, 600\right.$ $\mathrm{MHz}): \delta 7.400 \sim 7.358(\mathrm{~m}, 4 \mathrm{H}), 7.337 \sim 7.323(\mathrm{~m}, 2 \mathrm{H}), 7.238 \sim 7.221(\mathrm{~m}, 2 \mathrm{H}), 7.144 \sim 7.137$ (m, 4H), $7.120 \sim 7.097(\mathrm{~m}, 1 \mathrm{H}), 3.895(\mathrm{~s}, 2 \mathrm{H}), 2.739(\mathrm{~m}, \mathrm{~J}=6.6 \mathrm{~Hz}, 1 \mathrm{H}), 0.961(\mathrm{~d}, \mathrm{~J}=6.6 \mathrm{~Hz}$, $6 \mathrm{H}) .{ }^{13} \mathrm{C} \mathrm{NMR}\left(\mathrm{CDCl}_{3}, 150 \mathrm{MHz}\right): \delta 143.7,142.9,142.8,141.6,141.3,139.5,136.7,129.3$, $128.6,128.1,127.9,127.3,126.6,125.3,124.2,121.1,41.0,26.8,24.1$.

\section{General Procedure for $\mathrm{Pd}(\mathrm{OAc})_{2}$-Catalyzed Domino Carbopalladation-Cross-Coupling Reactions of Internal Alkynes with Hindered Grignard Reagents:}

In glove box with nitrogen atmosphere, to a mixture of alkyne $(0.5 \mathrm{mmol}), 1,2-$ dibromoethane $(1.5 \mathrm{mmol}, 130 \mu \mathrm{l}$ and $0.5 \mathrm{~mL}$ THF (in a schlenk flask) was added palladium acetate $(3.4 \mathrm{mg}, 0.015 \mathrm{mmol})$ and $\mathrm{PPh}_{3}(26.2 \mathrm{mg}, 0.1 \mathrm{mmol})$. After stirred for $5-10$ minutes, Grignard reagent $(2.0 \mathrm{~mL}, 1 \mathrm{M}$ in THF, $2.0 \mathrm{mmol})$ was added. The mixture was allowed to stir under refluxing for 20 hours. After quenched with water, the reaction mixture was extracted with ethyl acetate $(15 \mathrm{~mL} \times 3)$. The organic layer was washed with brine and the solvent was evaporated under vacuum. Flash chromatography on silica gel (hexane: ethyl acetate $=100$ : 0 to 90: 10) gave the cross-coupling products.

1,2-Bis(2,4,6-trimethylphenyl)stilbene (16): white solid. M. p.: 177-178.5 ${ }^{\circ} \mathrm{C} .{ }^{1} \mathrm{H} \mathrm{NMR}\left(\mathrm{CDCl}_{3}\right.$, $600 \mathrm{MHz}): \delta 7.047 \sim 7.025(\mathrm{~m}, 6 \mathrm{H}), 6.928 \sim 6.911(\mathrm{~m}, 4 \mathrm{H}), 6.641(\mathrm{~s}, 4 \mathrm{H}), 2.167(\mathrm{~s}, 6 \mathrm{H}), 2.052(\mathrm{~s}$, $12 \mathrm{H}) .{ }^{13} \mathrm{C} \mathrm{NMR}\left(\mathrm{CDCl}_{3}, 150 \mathrm{MHz}\right): \delta 142.812,140.323,138.228,136.729,135.893,131.150$, 128.595, 127.152, 125.828, 21.722, 20.890. Anal. calcd. for $\mathrm{C}_{32} \mathrm{H}_{32}$ : C, $92.26 \%$; $\mathrm{H}, 7.74 \%$. Found: C, 92.22\%; H, 7.78\%.

1,2-Bis(pentamethylphenyl)stilbene (17): ${ }^{5}$ white solid. m.p.: $252-254^{\circ} \mathrm{C} .{ }^{1} \mathrm{H} \mathrm{NMR}\left(\mathrm{CDCl}_{3}, 600\right.$ $\mathrm{MHz}): \delta 7.057 \sim 7.027(\mathrm{~m}, 6 \mathrm{H}), 7.000 \sim 6.987(\mathrm{~m}, 4 \mathrm{H}), 2.110(\mathrm{~s}, 12 \mathrm{H}), 2.105(\mathrm{~s}, 6 \mathrm{H}), 2.009(\mathrm{~s}$, $12 \mathrm{H}) .{ }^{13} \mathrm{C} \mathrm{NMR}\left(\mathrm{CDCl}_{3}, 150 \mathrm{MHz}\right): \delta 143.304,141.545,138.971,132.803,131.806,131.761$, $130.929,127.176,125.656,20.026,16.631,16.413$.

1,2-Bis(2,6-dimethylphenyl)-1,2-bis(4-methoxyphenyl)ethylene (18): white solid. M. p.: 204$206{ }^{\circ} \mathrm{C} .{ }^{1} \mathrm{H} \mathrm{NMR}\left(\mathrm{CDCl}_{3}, 600 \mathrm{MHz}\right): \delta 6.918(\mathrm{t}, \mathrm{J}=7.2 \mathrm{~Hz}, 2 \mathrm{H}), 6.873(\mathrm{~d}, \mathrm{~J}=9.0 \mathrm{~Hz}, 4 \mathrm{H}), 6.800$ $(\mathrm{d}, \mathrm{J}=7.2 \mathrm{~Hz}, 4 \mathrm{H}), 6.621(\mathrm{~d}, \mathrm{~J}=9.0 \mathrm{~Hz}, 4 \mathrm{H}), 3.748(\mathrm{~s}, 6 \mathrm{H}), 2.080(\mathrm{~s}, 12 \mathrm{H}) .{ }^{13} \mathrm{C} \mathrm{NMR}\left(\mathrm{CDCl}_{3}\right.$, $150 \mathrm{MHz}$ ): $\delta 157.589,141.201,138.863,136.904,135.054,132.133,127.587,126.457,112.713$, 55.076, 21.782. Anal. calcd. for $\mathrm{C}_{32} \mathrm{H}_{32} \mathrm{O}_{2}$ : C, 85.68\%; H, 7.19\%. Found: $\mathrm{C}, 85.38 \% ; \mathrm{H}, 7.16 \%$.

1,2-Bis(2,4,6-trimethylphenyl)-1,2-bis(4-methoxyphenyl)ethylene (19): white solid. M. p.: 195.5-196.5 ${ }^{\circ} \mathrm{C} .{ }^{1} \mathrm{H}$ NMR $\left(\mathrm{CDCl}_{3}, 600 \mathrm{MHz}\right): \delta 6.838$ (d, J=9.0 Hz, 4H), 6.627 (s, 4H), 6.598 (d, $\mathrm{J}=9.0 \mathrm{~Hz}, 4 \mathrm{H}), 3.737(\mathrm{~s}, 6 \mathrm{H}), 2.161(\mathrm{~s}, 6 \mathrm{H}), 2.029(\mathrm{~s}, 12 \mathrm{H}) .{ }^{13} \mathrm{C} \mathrm{NMR}\left(\mathrm{CDCl}_{3}, 50 \mathrm{MHz}\right): \delta$ 157.434, 138.892, 138.573, 136.715, 135.646, 135.585, 132.165, 128.540, 112.645, 55.079, 21.711, 20.900. Anal. calcd. for $\mathrm{C}_{34} \mathrm{H}_{36} \mathrm{O}_{2}$ : C, 85.67\%; H, 7.61\%. Found: $\mathrm{C}, 85.79 \%$; H, 7.64\%. 
3,4-Bis(2,4,6-trimethylphenyl)hex-3-ene (20): ${ }^{5}$ white solid. M. p.: $165-166^{\circ} \mathrm{C} .{ }^{1} \mathrm{H}$ NMR $\left(\mathrm{CDCl}_{3}, 600 \mathrm{MHz}\right): \delta 6.648(\mathrm{~s}, 4 \mathrm{H}), 2.472(\mathrm{q}, \mathrm{J}=7.2 \mathrm{~Hz}, 4 \mathrm{H}), 2.154(\mathrm{~s}, 6 \mathrm{H}), 2.064(\mathrm{~s}, 12 \mathrm{H})$, $1.016(\mathrm{t}, \mathrm{J}=7.2 \mathrm{~Hz}, 6 \mathrm{H}) .{ }^{13} \mathrm{C}$ NMR $\left(\mathrm{CDCl}_{3}, 150 \mathrm{MHz}\right): \delta 138.615,138.471,135.697,134,880$, $128.083,28.228,20.926,20.803,13.382$.

3,4-Bis(pentamethylphenyl)hex-3-ene (21): ${ }^{5}$ white solid. M. p.: $175-177^{\circ} \mathrm{C} .{ }^{1} \mathrm{H}$ NMR $\left(\mathrm{CDCl}_{3}\right.$, $600 \mathrm{MHz}): \delta 2.498(\mathrm{q}, \mathrm{J}=7.2 \mathrm{~Hz}, 4 \mathrm{H}), 2.112(\mathrm{~s}, 6 \mathrm{H}), 2.025(\mathrm{~s}, 12 \mathrm{H}), 2.004(\mathrm{~s}, 12 \mathrm{H}), 1.059$ (t, J= $7.2 \mathrm{~Hz}, 6 \mathrm{H}) .{ }^{13} \mathrm{C} \mathrm{NMR}\left(\mathrm{CDCl}_{3}, 150 \mathrm{MHz}\right): \delta 139.449,139.330,131.965,131.600,131.112$, $29.343,19.647,16.544,16.442,13.272$.

1,2-Bis(2,6-dimethylphenyl)prop-1-ene (22): white solid. m.p.: 120-121 ${ }^{\circ} \mathrm{C} .{ }^{1} \mathrm{H}$ NMR $\left(\mathrm{CDCl}_{3}\right.$, $600 \mathrm{MHz}$ ): $\delta 7.295$ (d, J= 4.2 Hz, 4H), 7.192 (q, J= 4.2 Hz, 1H), 6.929 (t, J= $7.8 \mathrm{~Hz}, 1 \mathrm{H}), 6.890$ $(\mathrm{t}, \mathrm{J}=7.8 \mathrm{~Hz}, 1 \mathrm{H}), 6.852(\mathrm{~d}, \mathrm{~J}=7.8 \mathrm{~Hz}, 2 \mathrm{H}), 6.774(\mathrm{~d}, \mathrm{~J}=7.8 \mathrm{~Hz}, 2 \mathrm{H}), 2.216(\mathrm{~s}, 3 \mathrm{H}), 2.202(\mathrm{~s}$, $6 \mathrm{H}), 2.084(\mathrm{~s}, 6 \mathrm{H}) .{ }^{13} \mathrm{C}$ NMR $\left(\mathrm{CDCl}_{3}, 150 \mathrm{MHz}\right): \delta 141.991,141.966,140.920,137.862$, $136.641,135.777,135.257,130.090,127.404,127.383,127.355,126.316,126.102,126.032$, $22.750,21.778,21.068$. The stereochemistry of 1,2-bis(dimethylphenyl)prop-1-ene was established by NOE effect: NOE effect observed when irradiated at the peak at $\delta 2.084 \mathrm{ppm}$ : 7.295 (d, 0.33\%), 6.774 (d, 0.32\%), 2.202 (s, 0.75\%). Anal. calcd. for $\mathrm{C}_{25} \mathrm{H}_{26}: \mathrm{C}, 91.97 \% ; \mathrm{H}$, $8.03 \%$. Found: C, $91.41 \%$; H, 7.94\%.

1,2-Bis(2,4,6-trimethylphenyl)prop-1-ene (23): white solid. M. p.: $133-135^{\circ} \mathrm{C} .{ }^{1} \mathrm{H}$ NMR $\left(\mathrm{CDCl}_{3}, 600 \mathrm{MHz}\right): \delta 7.275 \sim 7.259(\mathrm{~m}, 4 \mathrm{H}), 7.181 \sim 7.153(\mathrm{~m}, 1 \mathrm{H}), 6.682(\mathrm{~s}, 2 \mathrm{H}), 6.598(\mathrm{~s}, 2 \mathrm{H})$, $2.173(\mathrm{~s}, 3 \mathrm{H}), 2.165(\mathrm{~s}, 3 \mathrm{H}), 2.162(\mathrm{~s}, 6 \mathrm{H}), 2.132(\mathrm{~s}, 3 \mathrm{H}), 2.041(\mathrm{~s}, 6 \mathrm{H}) .{ }^{13} \mathrm{C} \mathrm{NMR}\left(\mathrm{CDCl}_{3}, 150\right.$ MHz): $\delta 142.475,139.287,138.273,137.883,136.416,135.686,135.454,135.296,135.106$, 130.086, 128.320, 128.306, 127.292, 125.842, 23.020, 21.697, 20.988, 20.837, 20.819. Anal. calcd. for $\mathrm{C}_{27} \mathrm{H}_{30}$ : C, $91.47 \%$; $\mathrm{H}, 8.53 \%$. Found: $\mathrm{C}, 91.58 \%$; $\mathrm{H}, 8.52 \%$.

1,2-Bis(pentamethylphenyl)prop-1-ene (24): white solid. M. p.: $177-179{ }^{\circ} \mathrm{C} .{ }^{1} \mathrm{H}$ NMR $\left(\mathrm{CDCl}_{3}\right.$, $600 \mathrm{MHz}$ ): $\delta 7.329$ (d, J=7.2 Hz, 2H), 7.275 (t, J=7.2 Hz, 2H), 7.156 (t, J=7.2 Hz, 1H), 2.219 (s, 3H), 2.123(s, 6H), 2.118 (s, 3H), 2.085 (s, 3H), 2.073 (s, 6H), $2.053(\mathrm{~s}, 6 \mathrm{H}), 1.996(\mathrm{~s}, 6 \mathrm{H})$. ${ }^{13} \mathrm{C}$ NMR $\left(\mathrm{CDCl}_{3}, 150 \mathrm{MHz}\right): \delta$ 143.063, 140.114, 138.966, 138.927, 137.112, 132.447, 132.362, 131.962, 131.716, 131.615, 130.375, 129.933, 127.307, 125.598, 24.145, 20.062, 19.641, 16.611, $16.513,16.453,16.380$. The stereochemistry of 1,2-bis(pentamethylphenyl)prop-1-ene was established by NOE effect: NOE effect observed when irradiated at the peak at $\delta 2.219 \mathrm{ppm}$ : 7.329 (d, $0.7 \%), 2.123(\mathrm{~s}, 1.0 \%)$.

\section{Reference:}

1. Miller, A.R.; Curtin D.Y. J. Am. Chem. Soc. 1976, 98, 1860-1865.

2. Hawkins, R. T.; Lennarz, W. J.; Snyder, H. R. J. Am. Chem. Soc. 1960, 82, 3053-3059.

3. Furniss, B.S.; Hannaford, A. J.; Smith, P.W.G.; Tatchell, A.R. Vogel's Textbook of Practical Organic Chemistry ( $5^{\text {th }}$ ed.), 1989, P933-935.

4. Sonogashira, K.; Tohda, Y.; Hagihara, N. Tetrahedron Lett. 1975, 4467.

5. Rathore, R.; Deselnicu, M. I.; Burns, C. L. J. Am. Chem. Soc. 2002, 124, 14832-14833. 


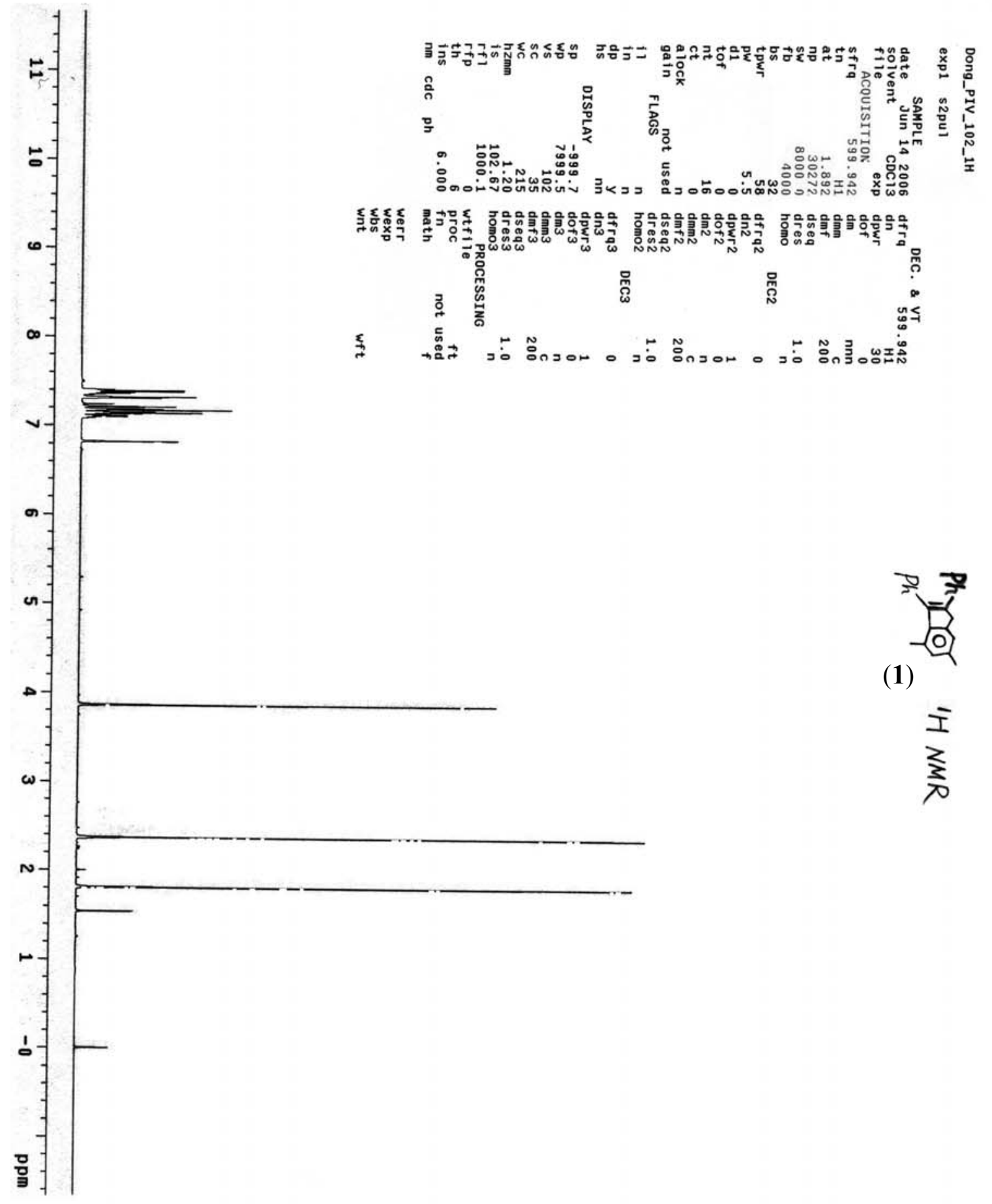




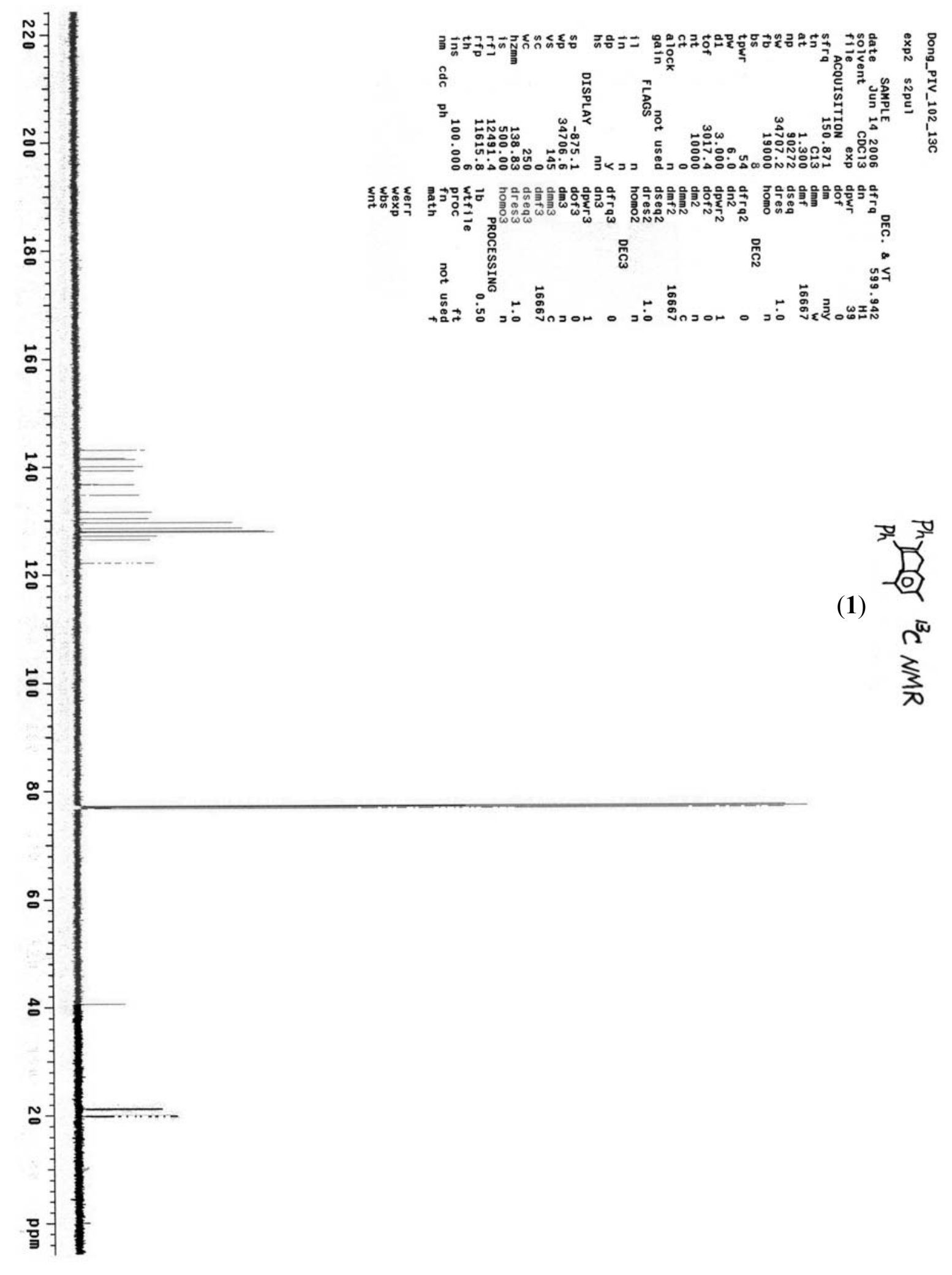



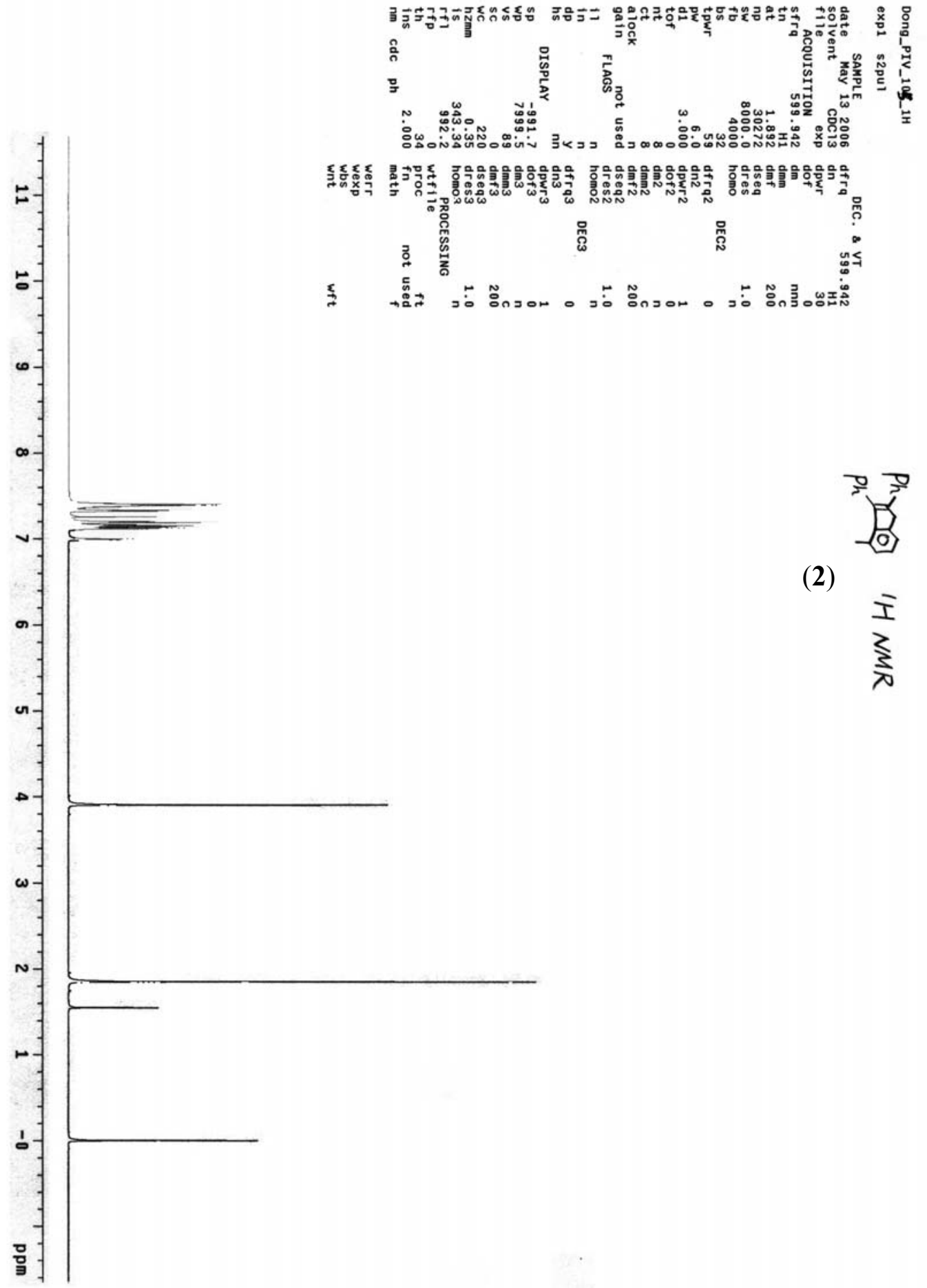

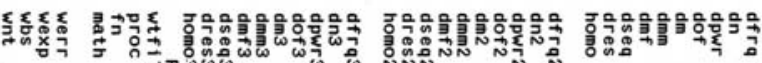



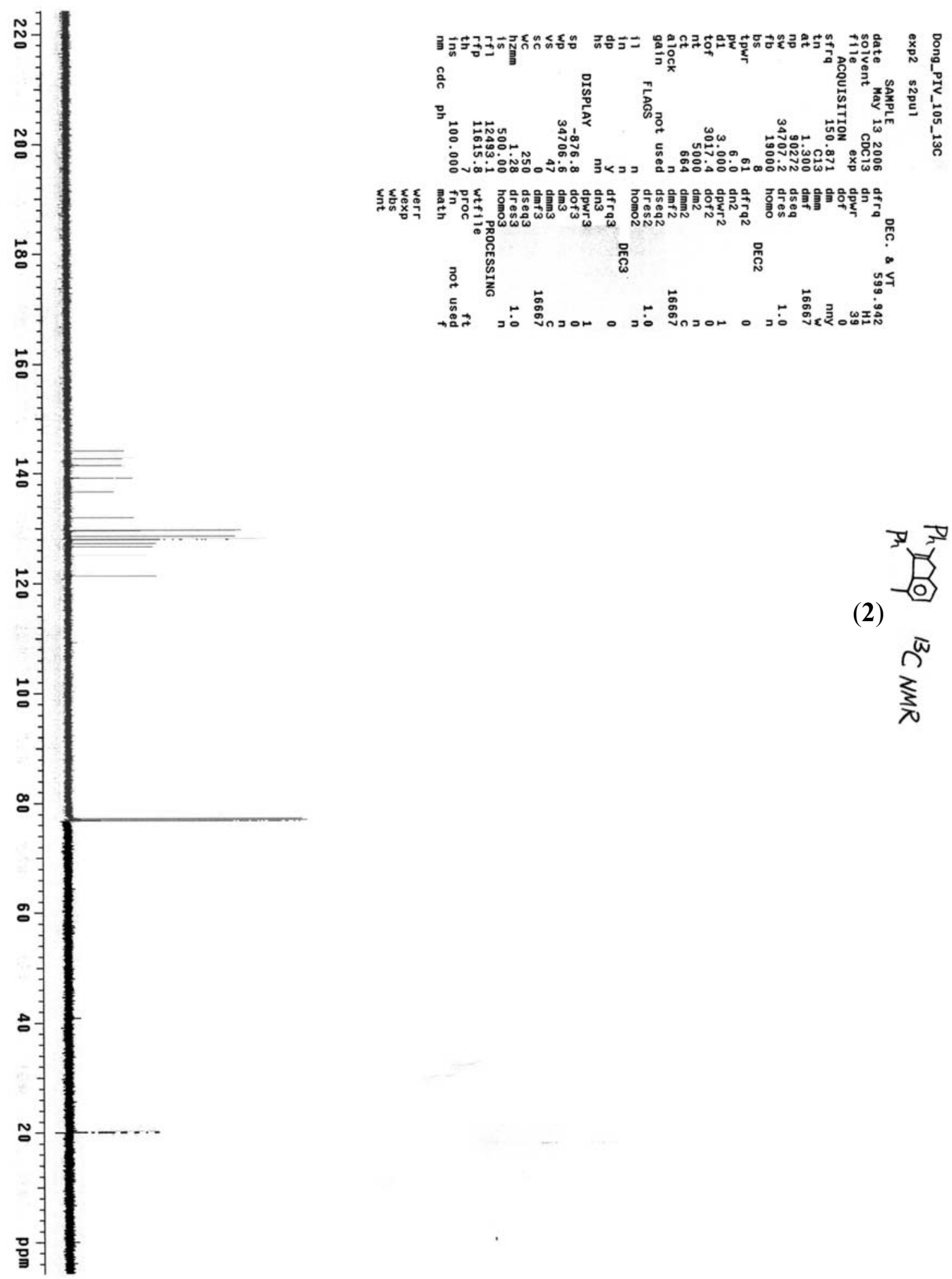

(2)

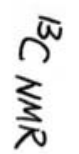



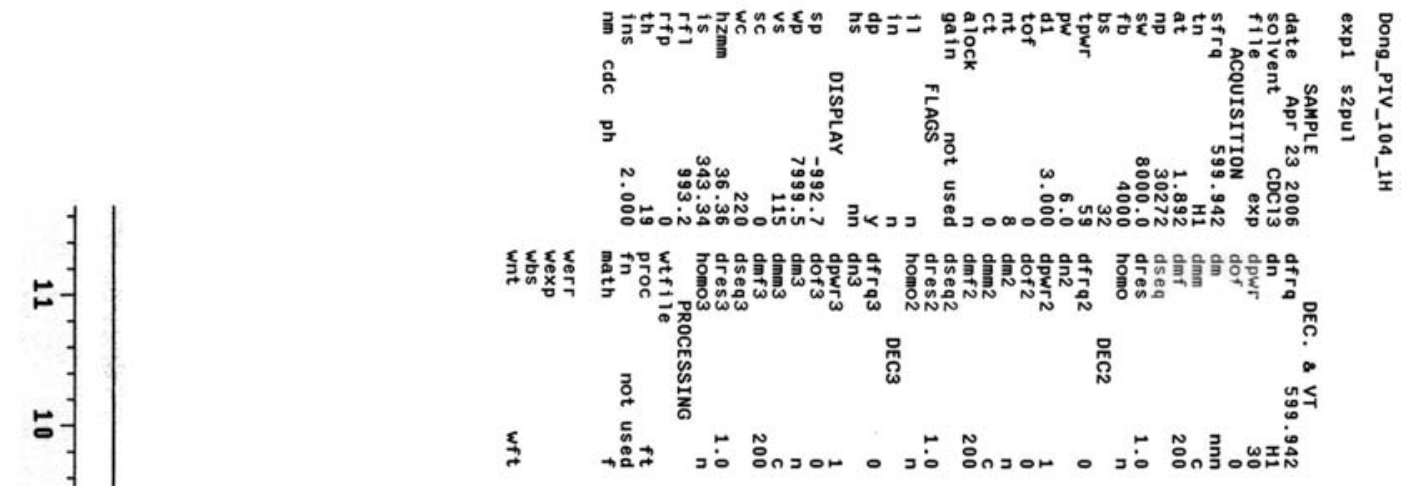

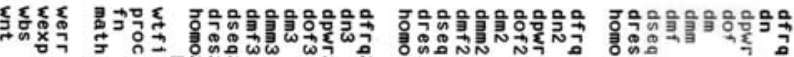

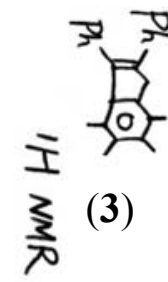

목

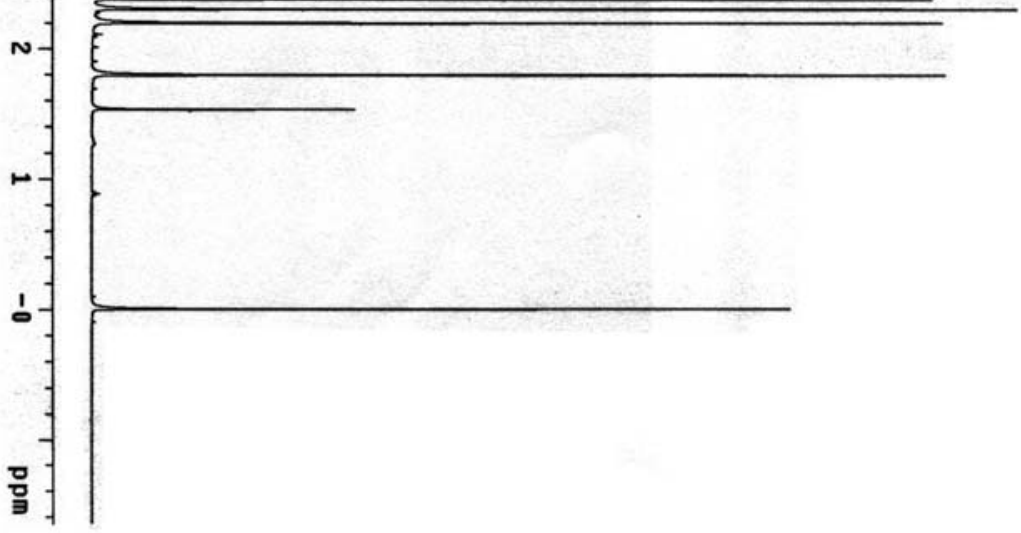




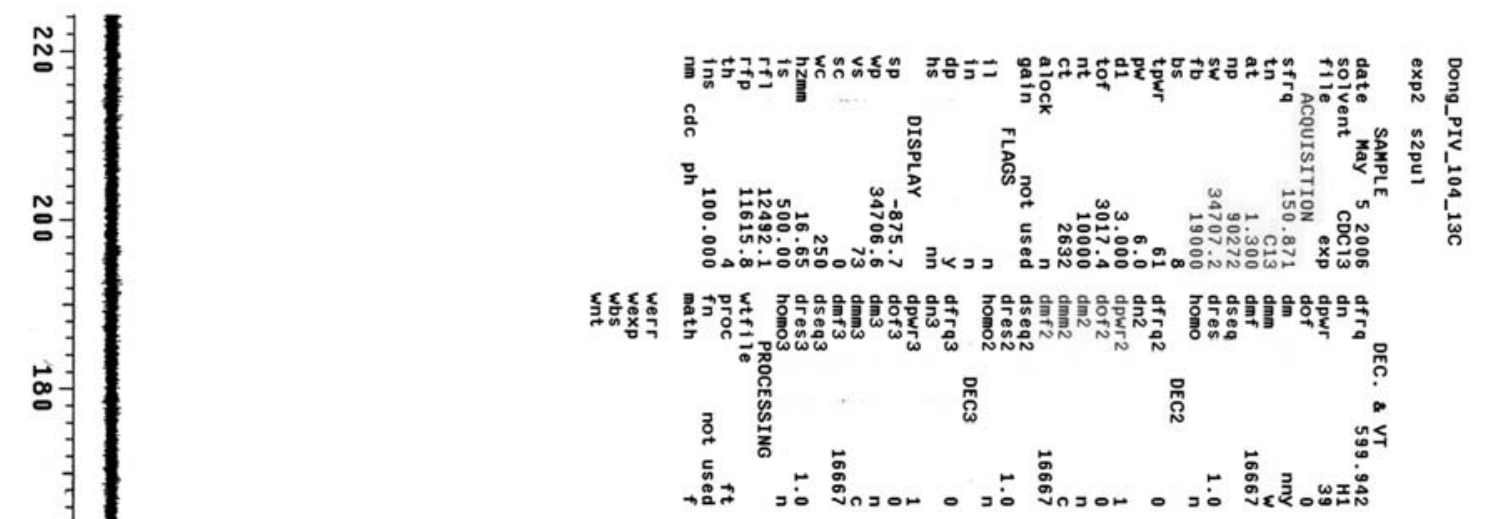

응

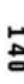
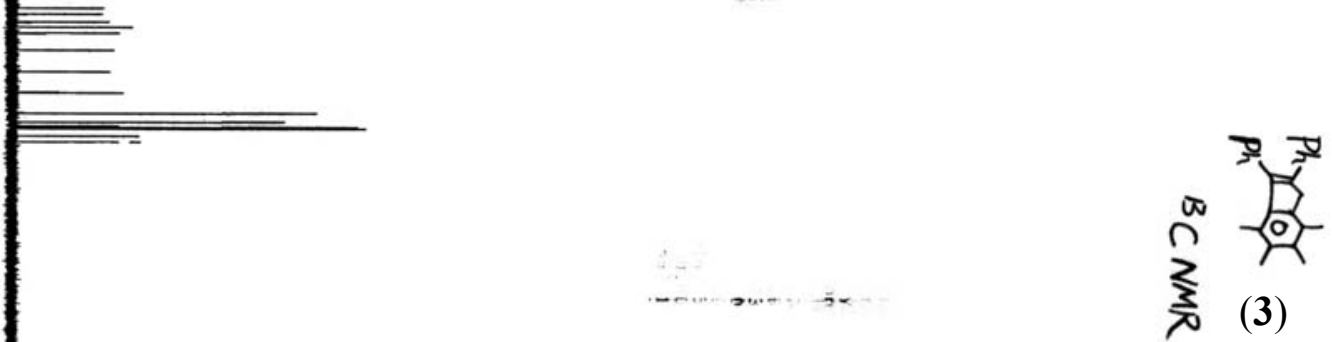

$\stackrel{\circ}{\circ}$

$\stackrel{0}{\circ}$
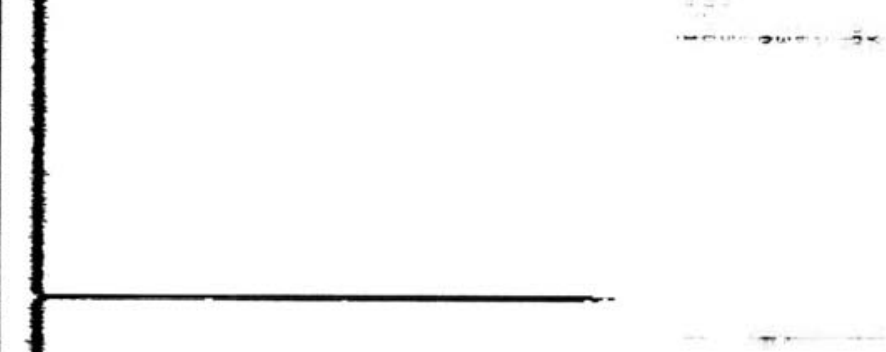

제 (3)

:-

ㅇ-

.

걱

뭅

S- 12 

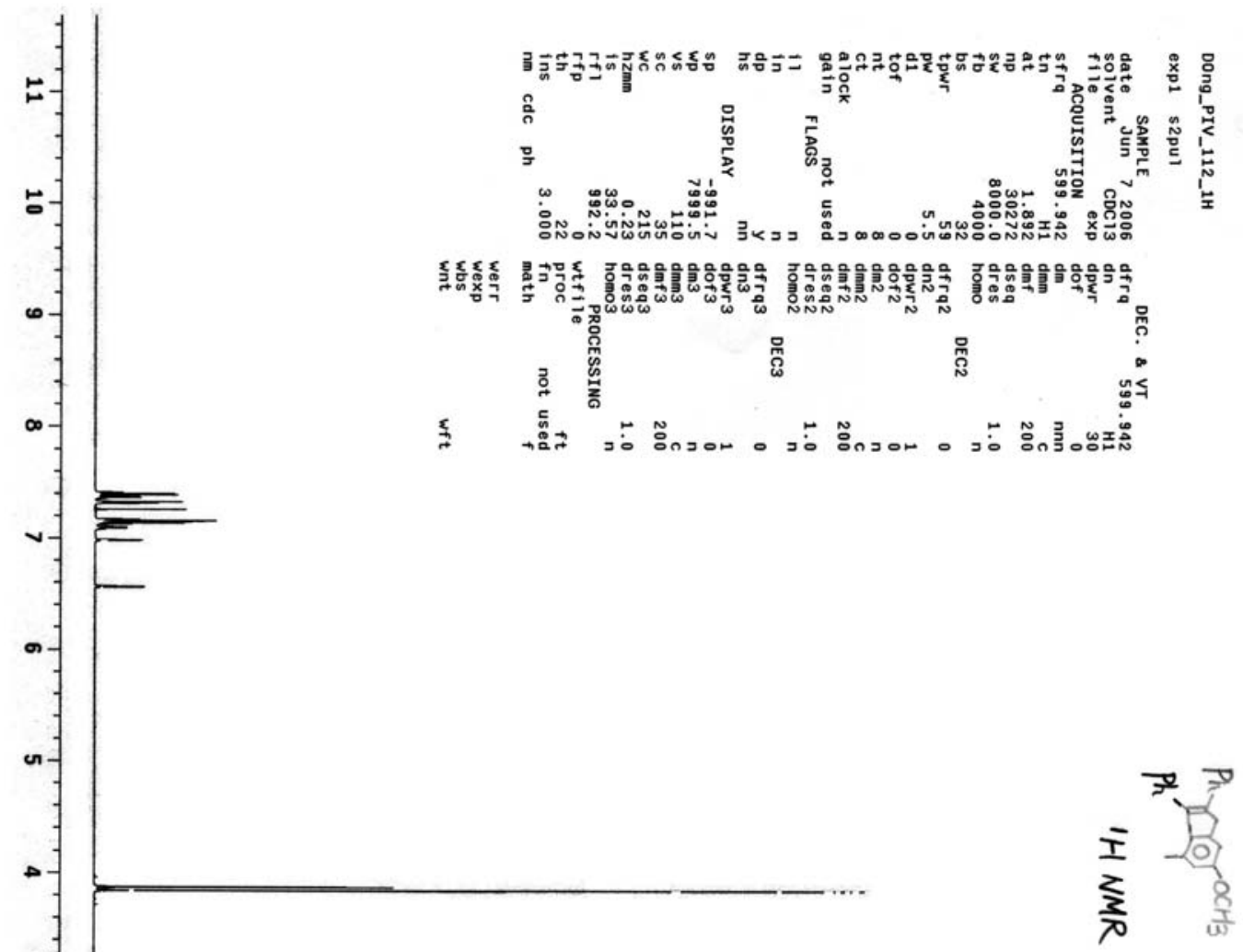

(4)

믑

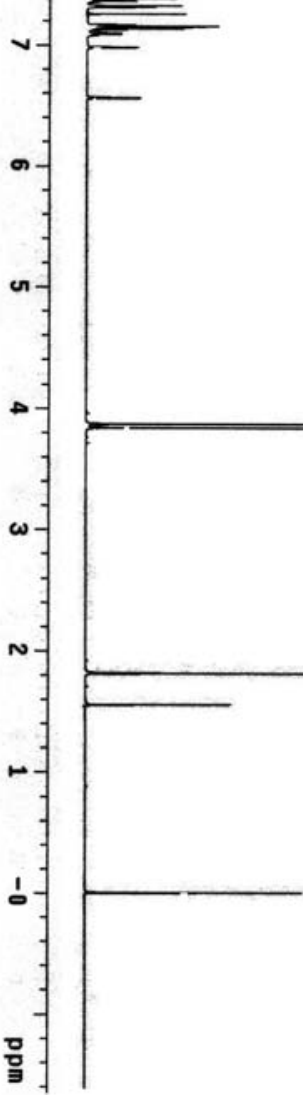

S- 13 

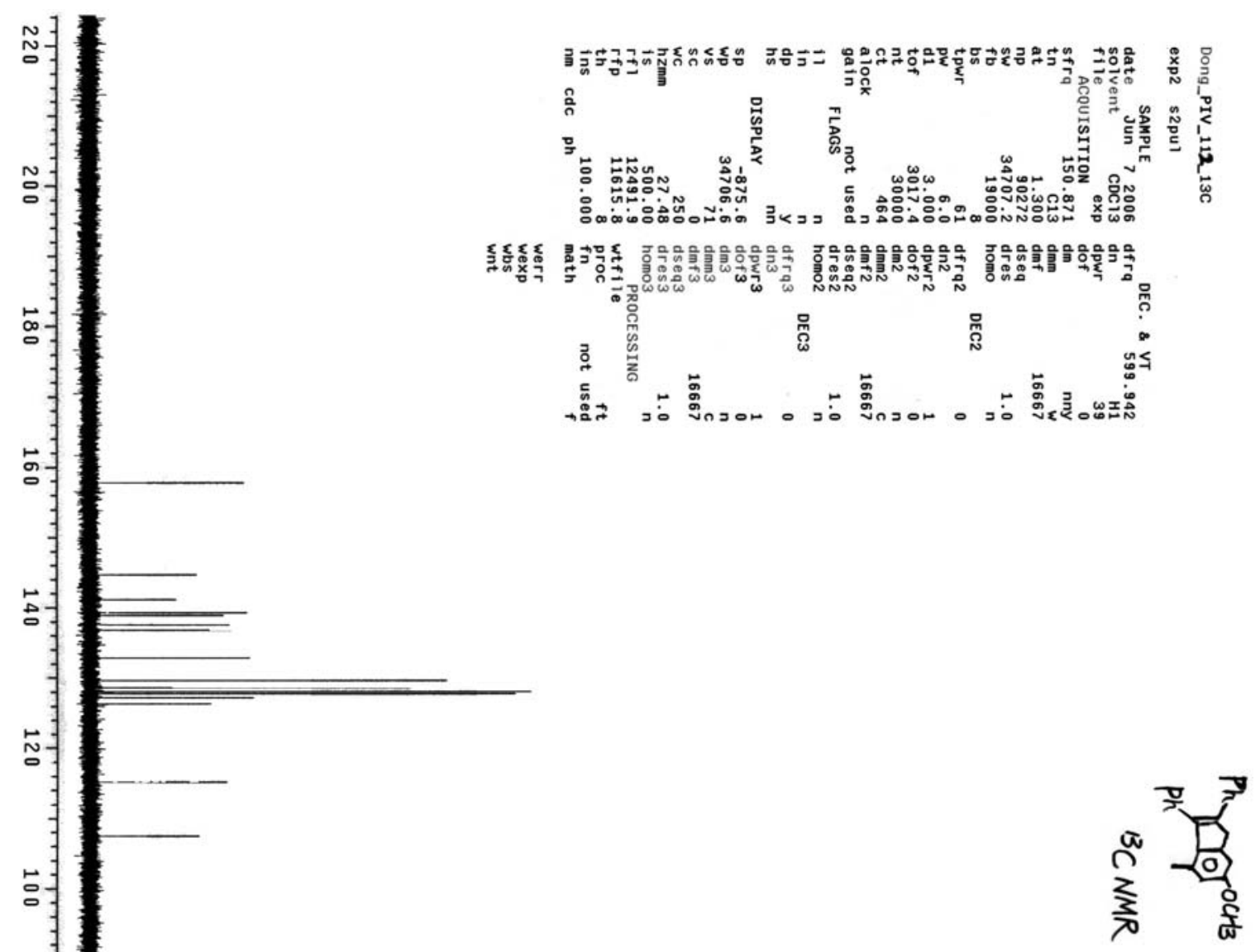

(4) 

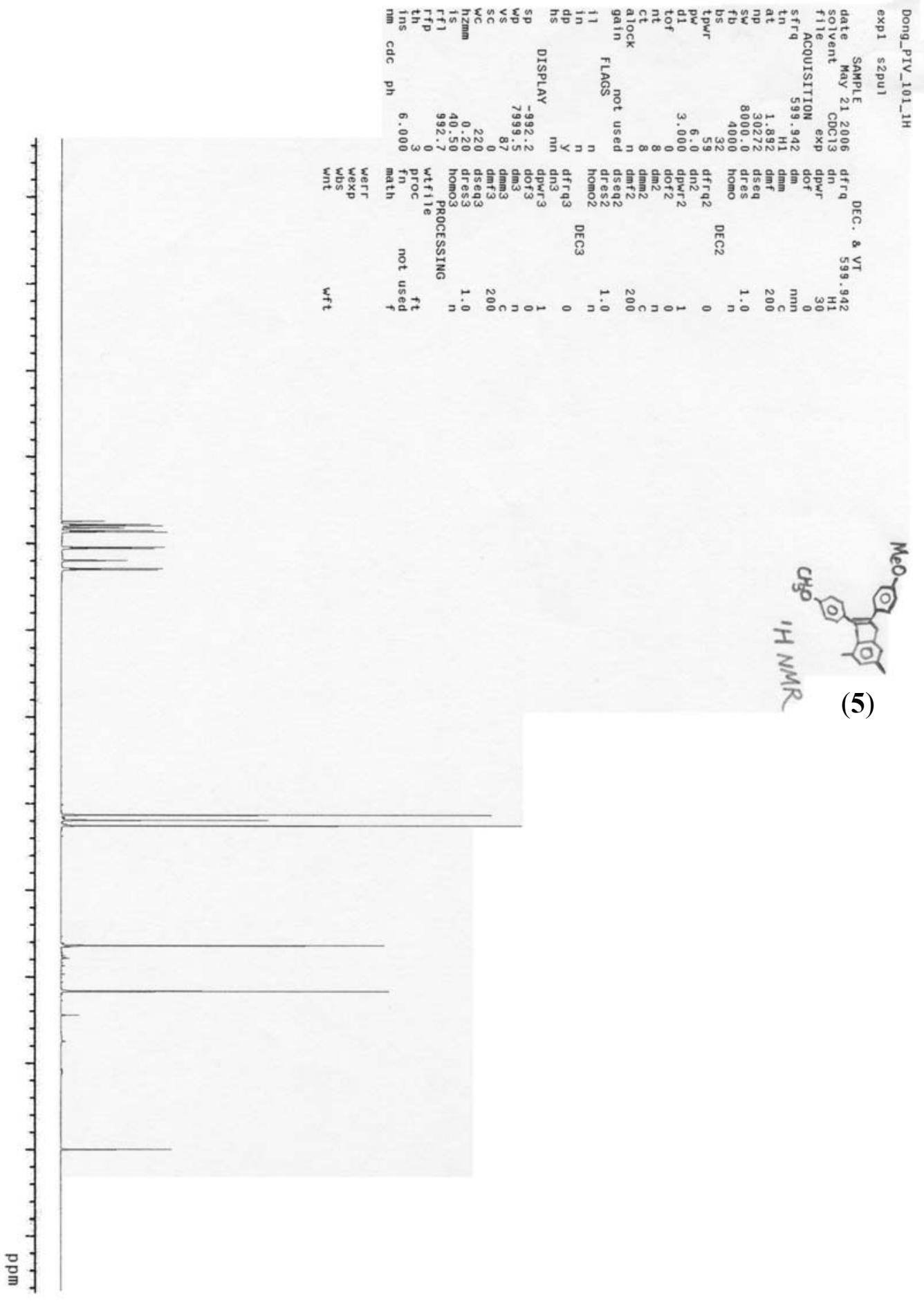

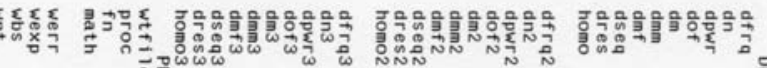

亚

S- 15 

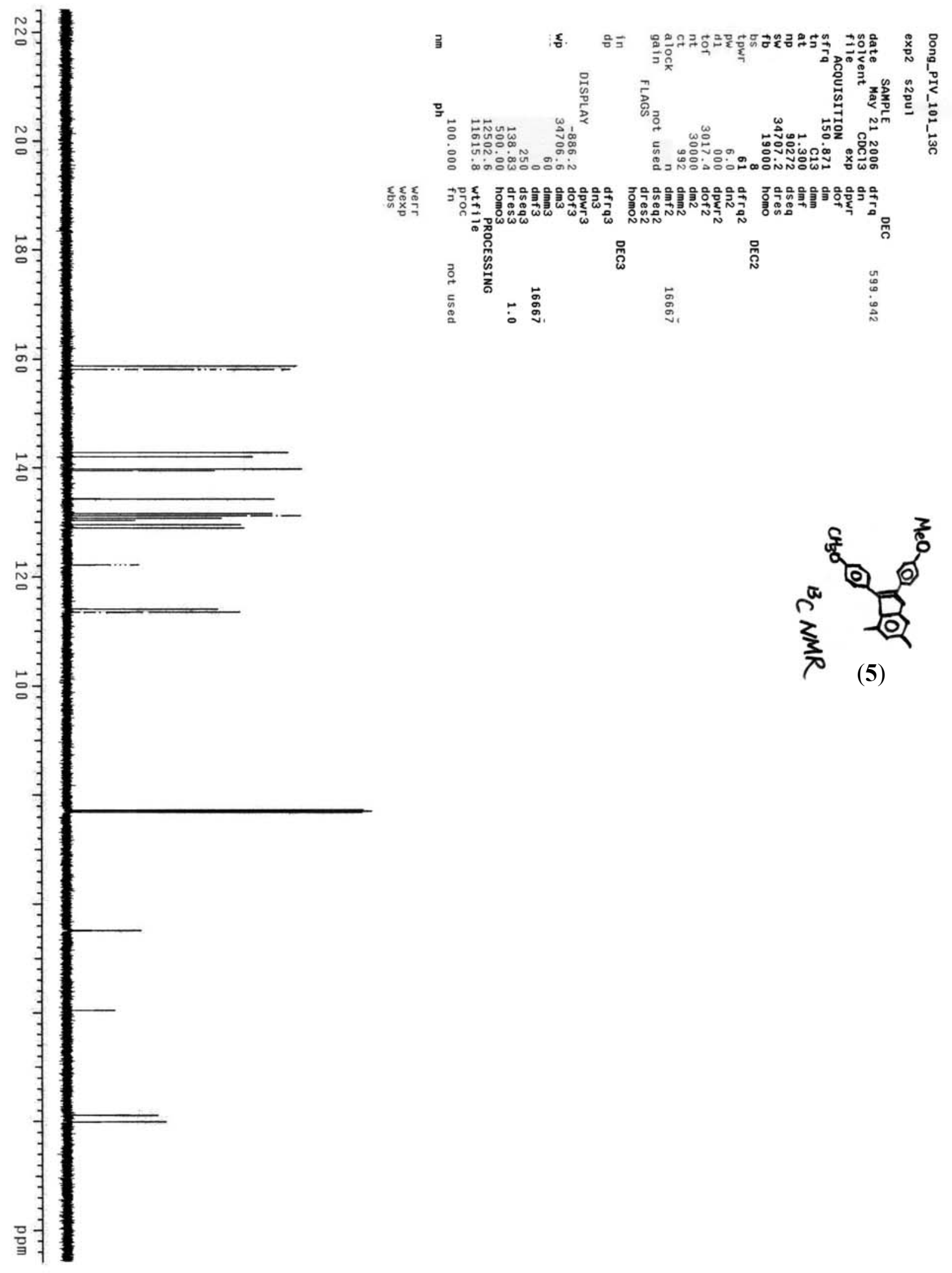

S- 16 


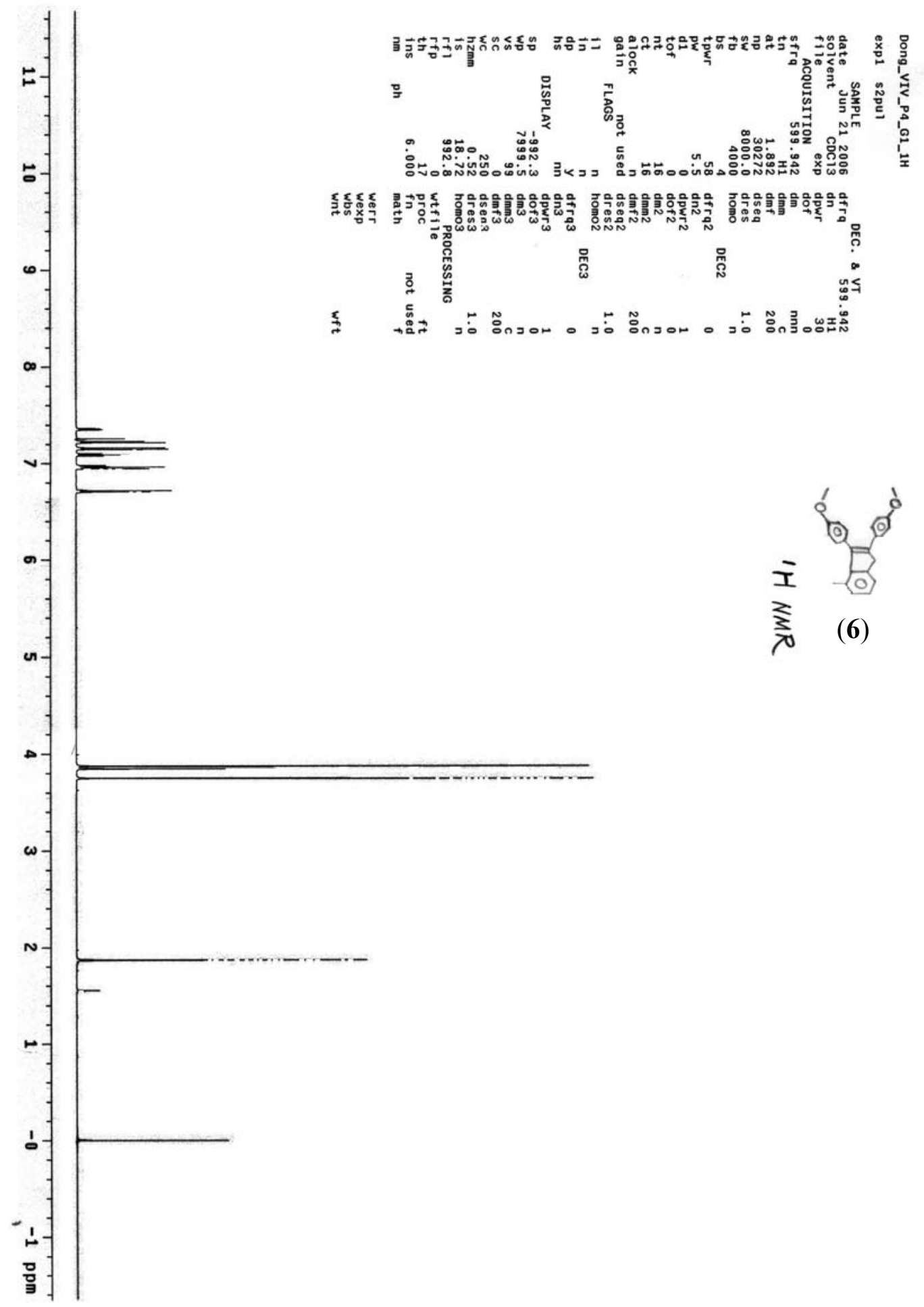

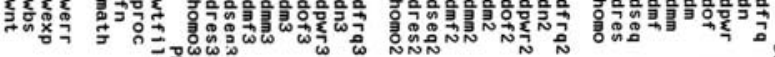

S- 17 

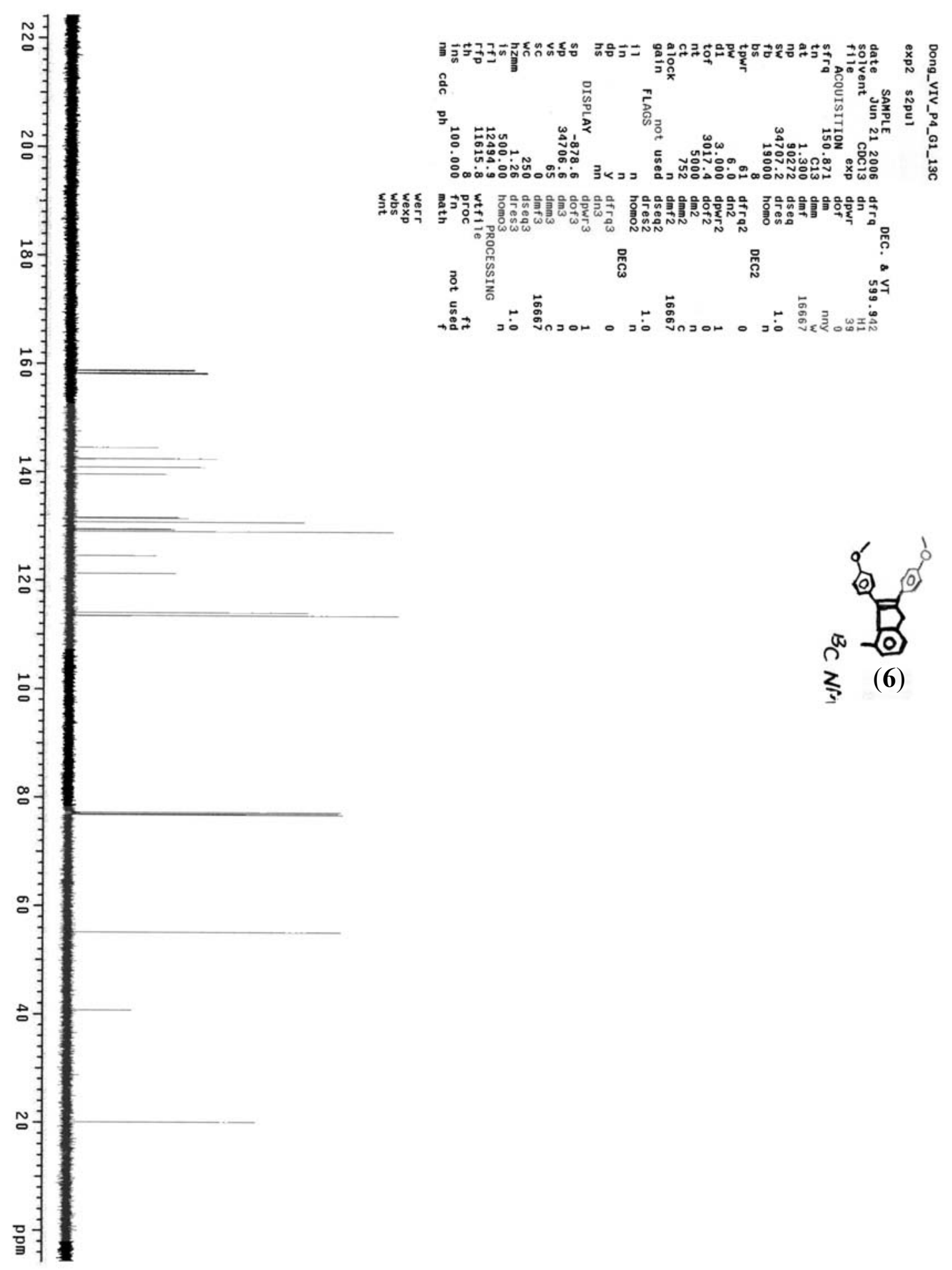

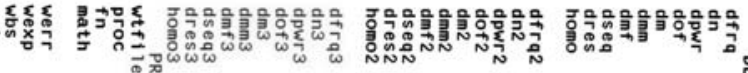

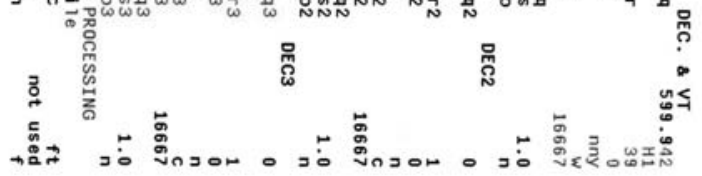




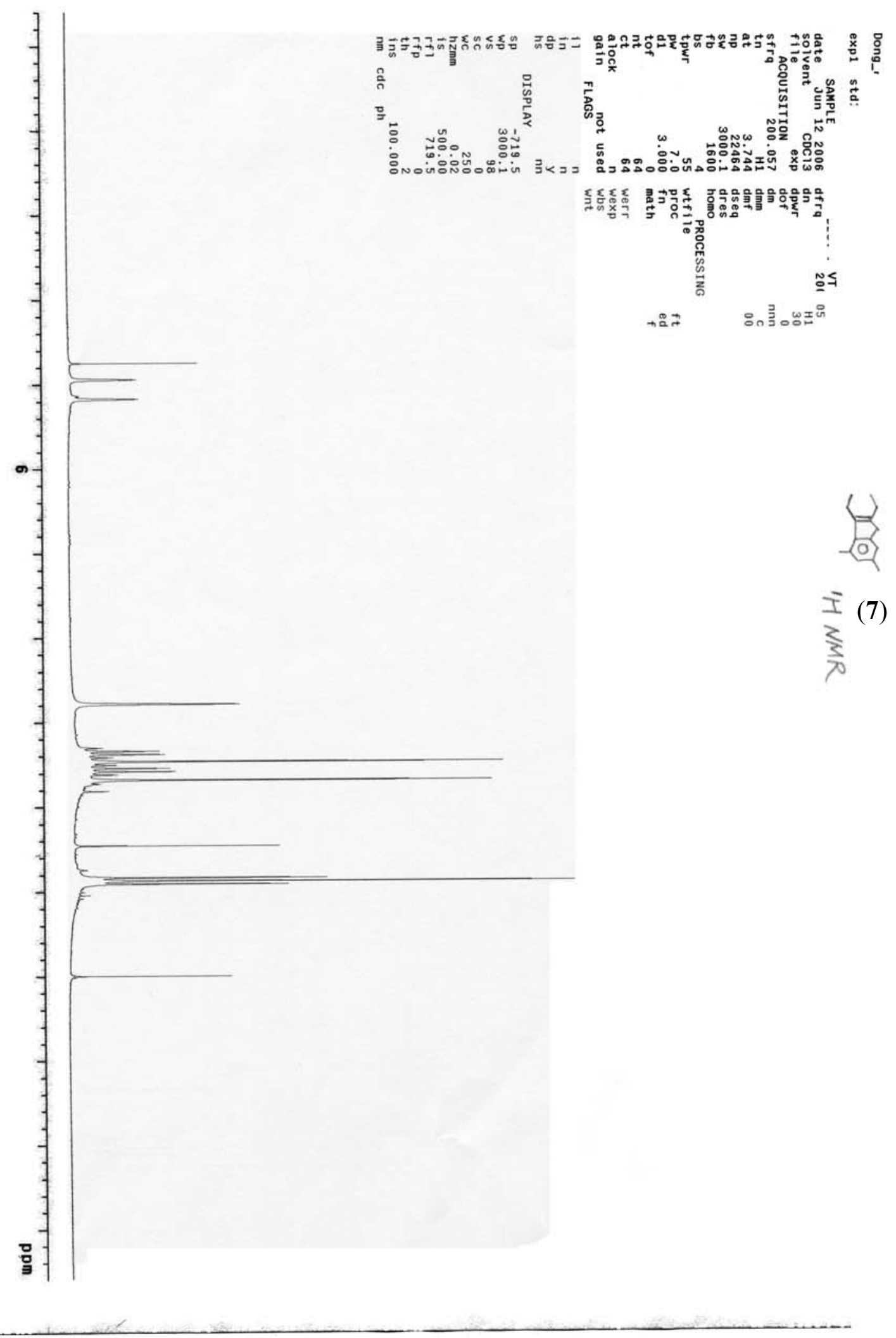

S- 19 

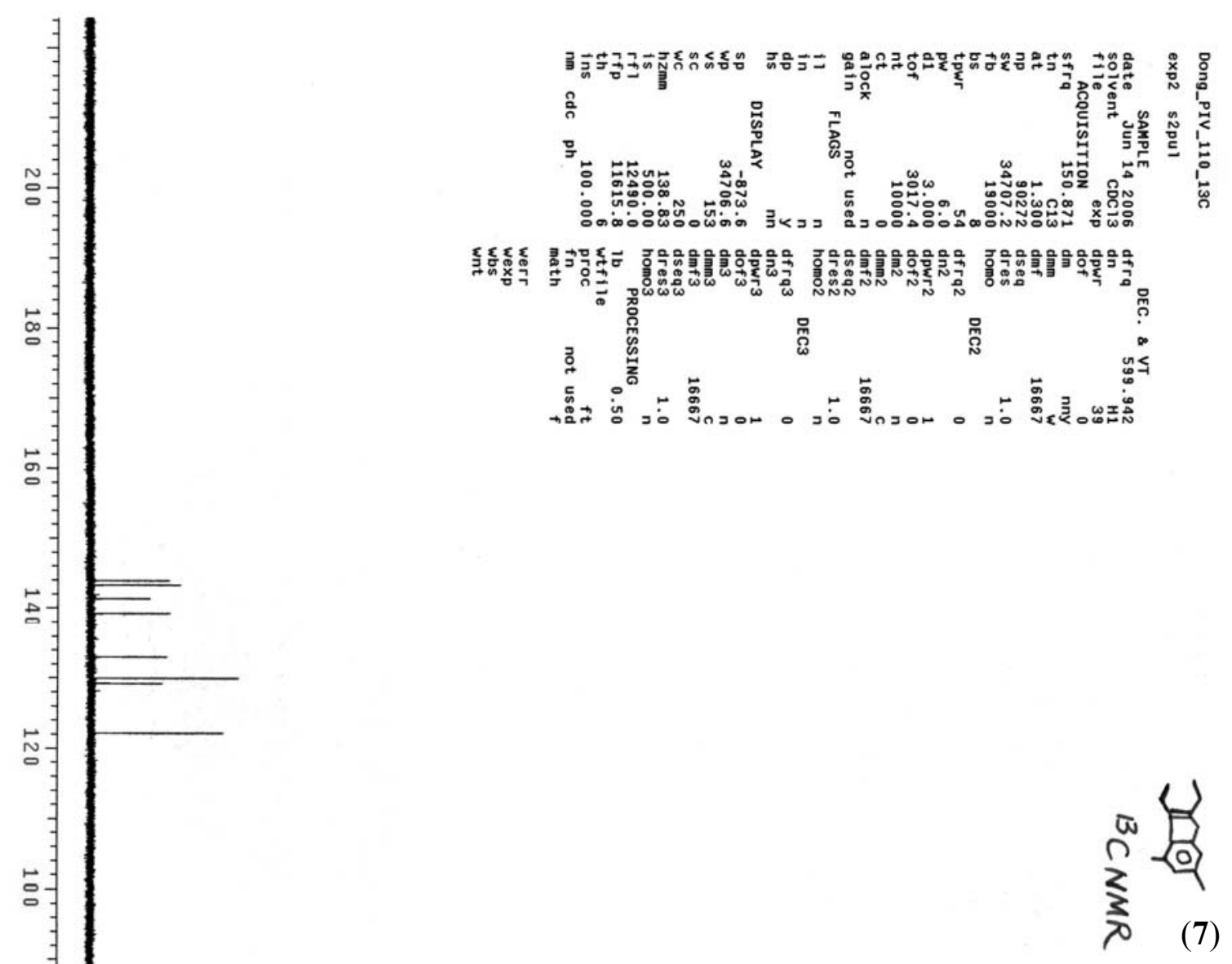

뭌 


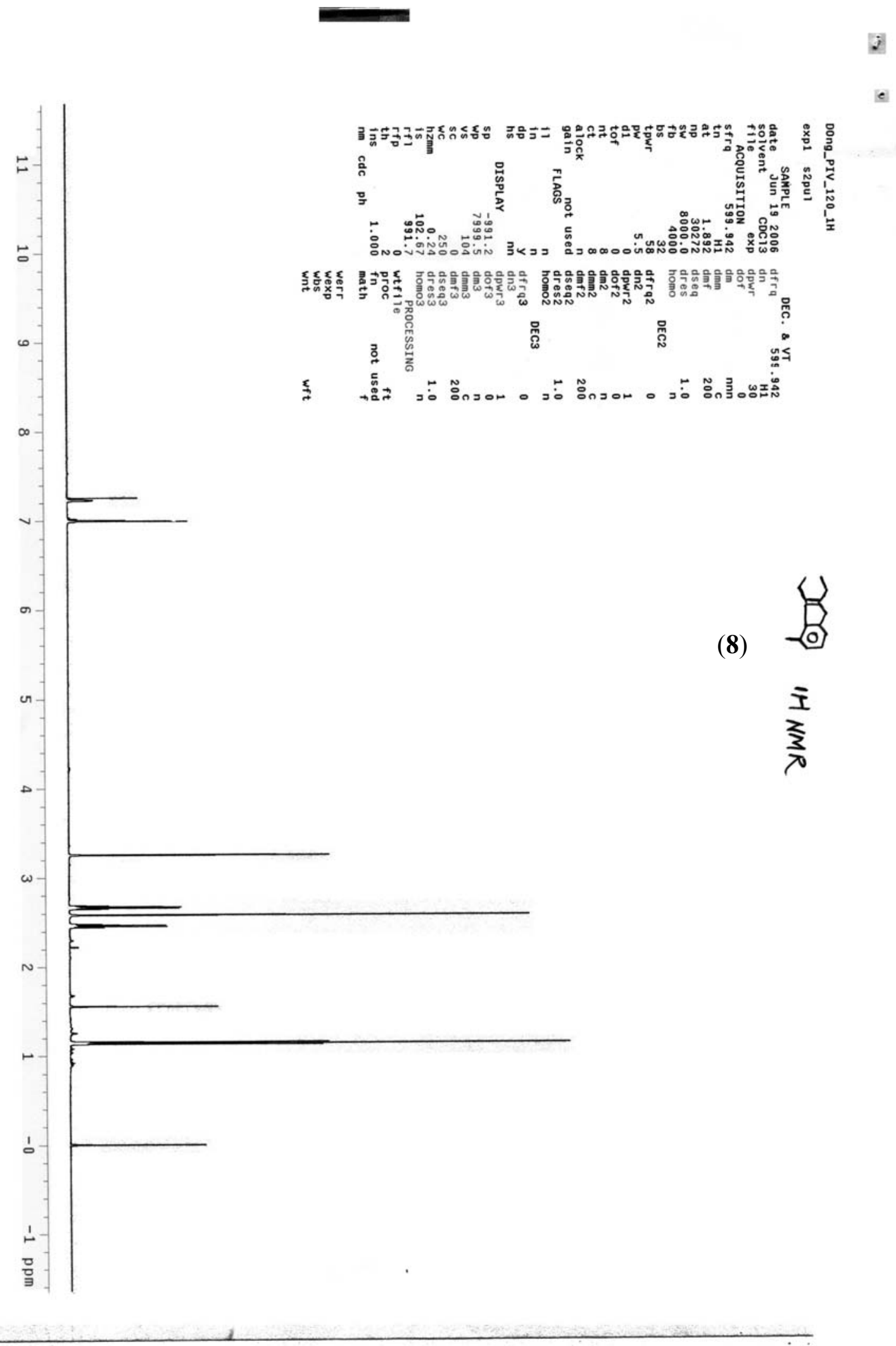

S- 21 

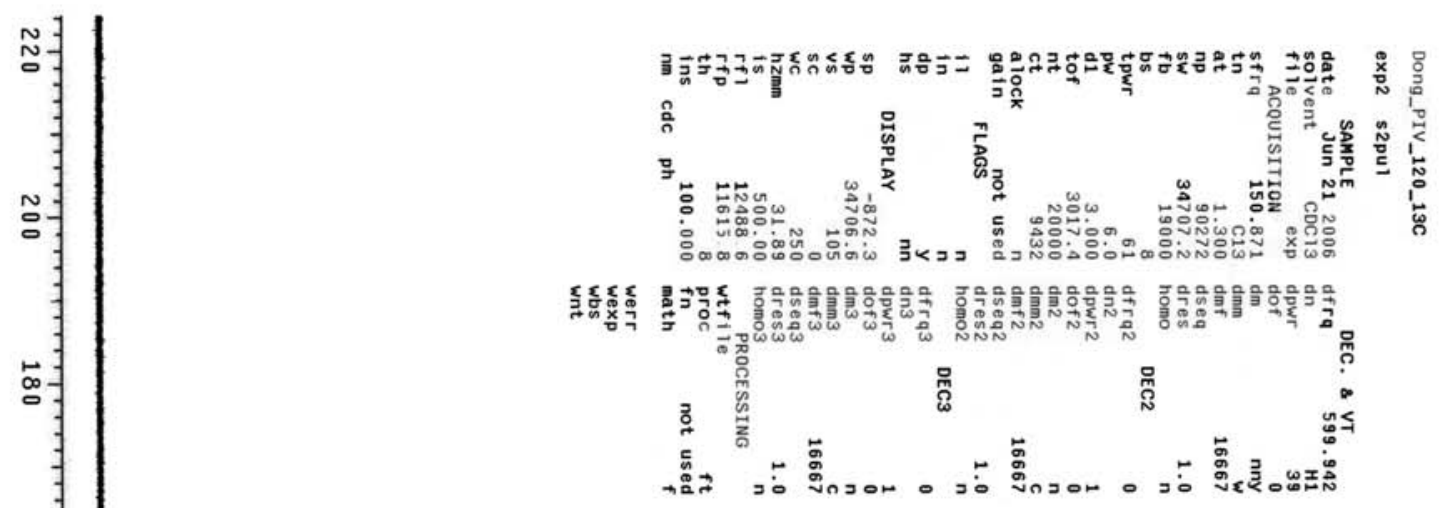

。

$\stackrel{\infty}{\infty}$

.

宫:

䚄

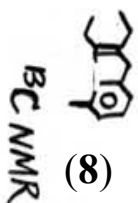

뭌국

S- 22 


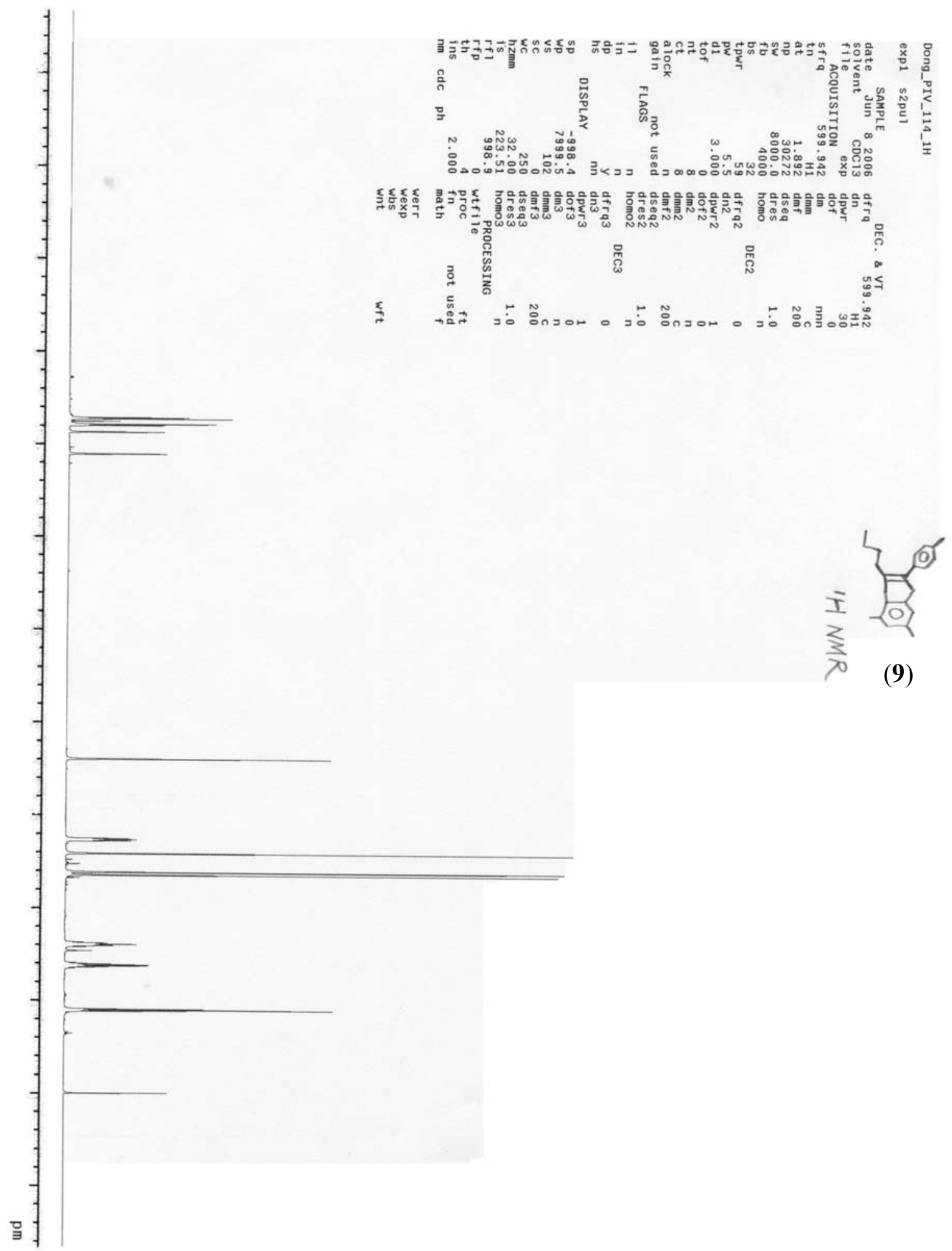

S- 23 

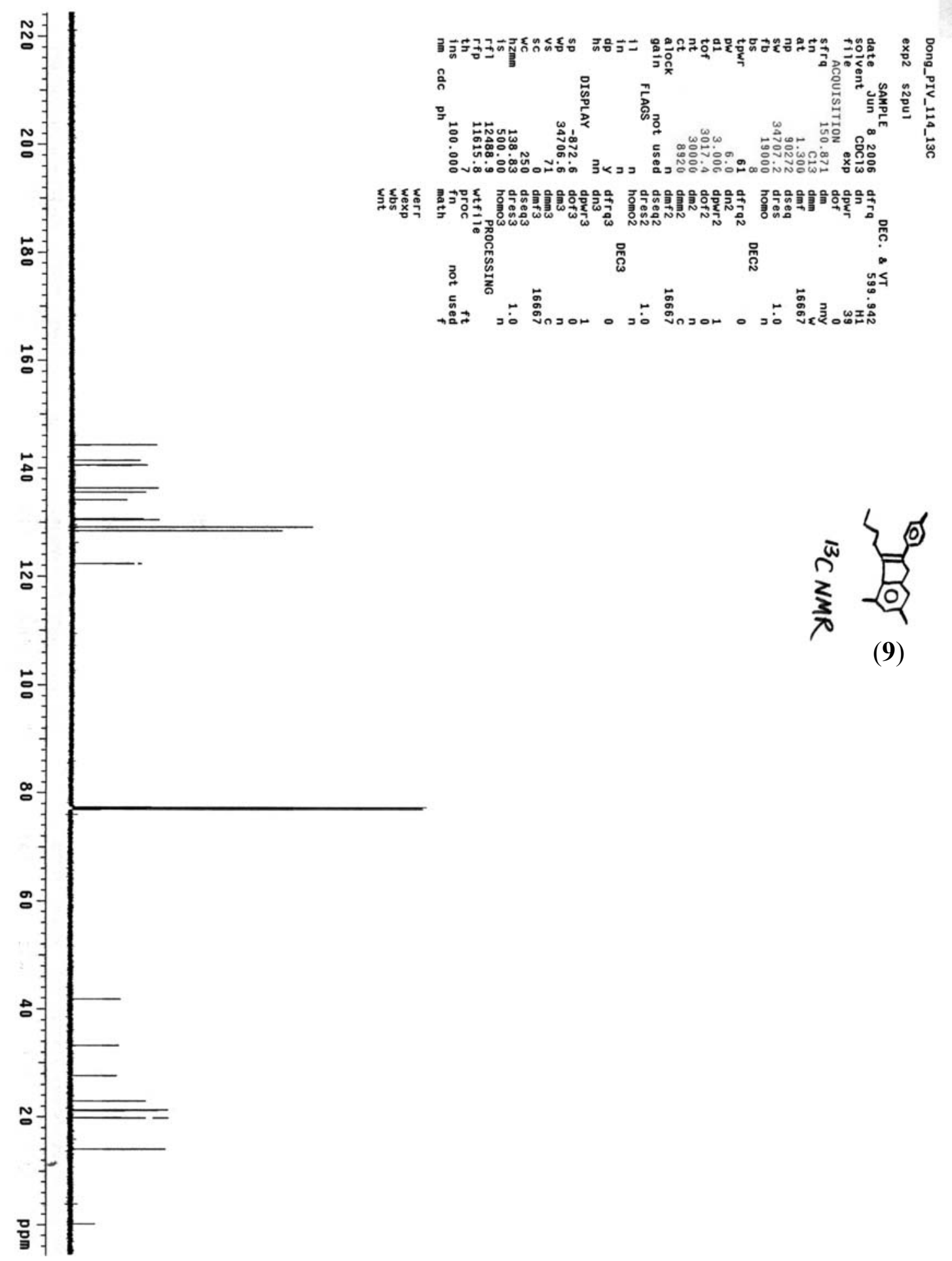

(9) 

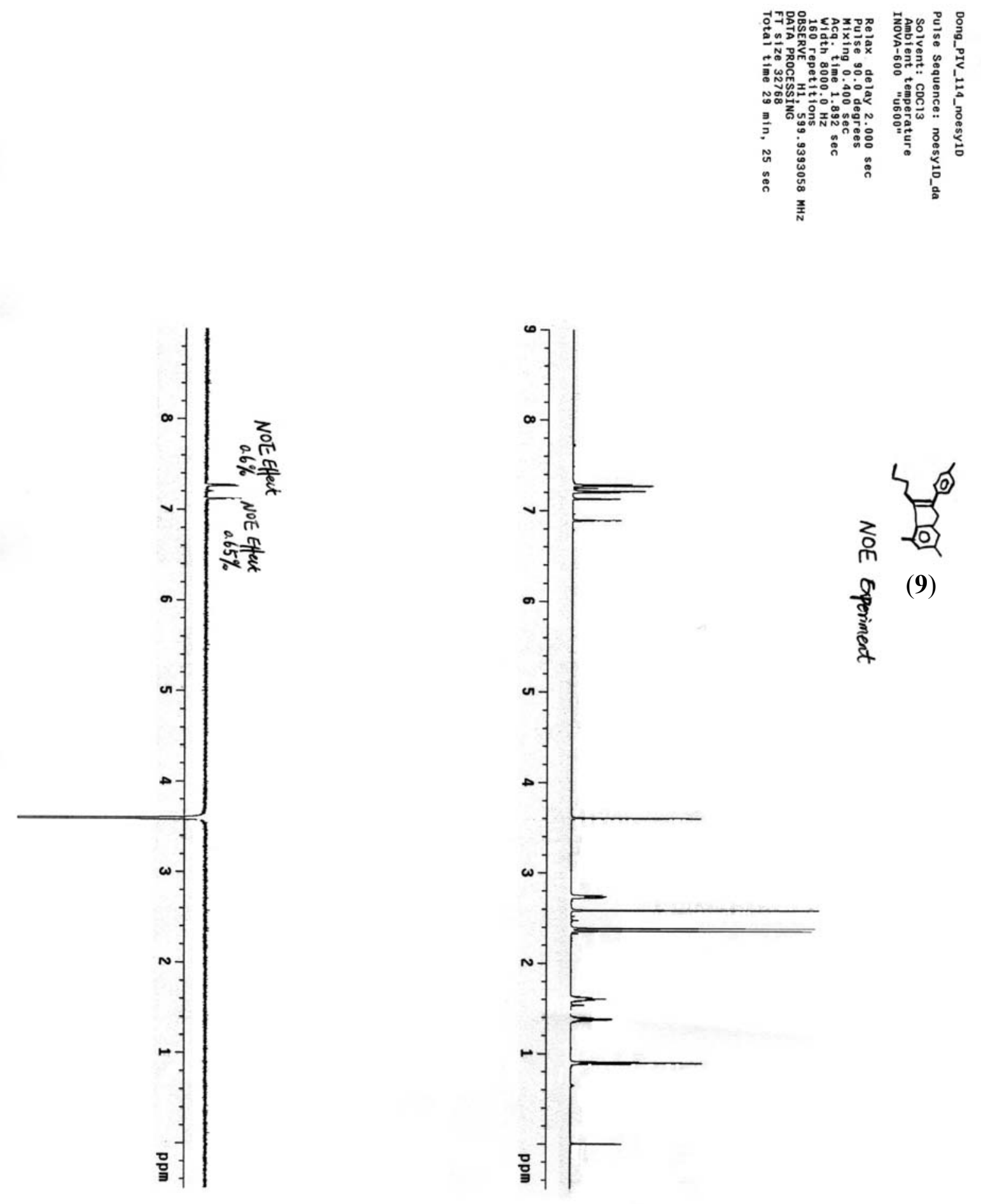


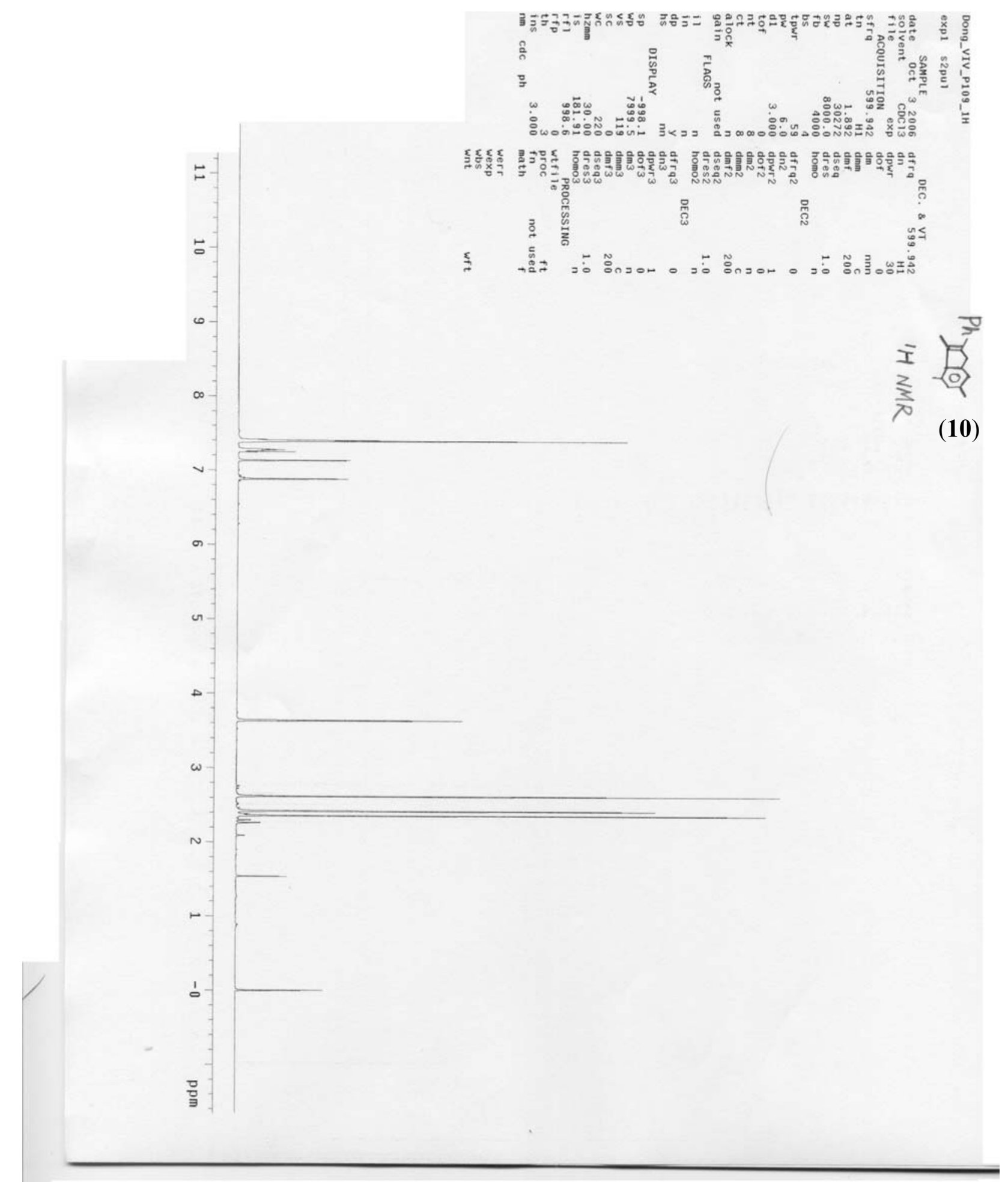

S- 26 


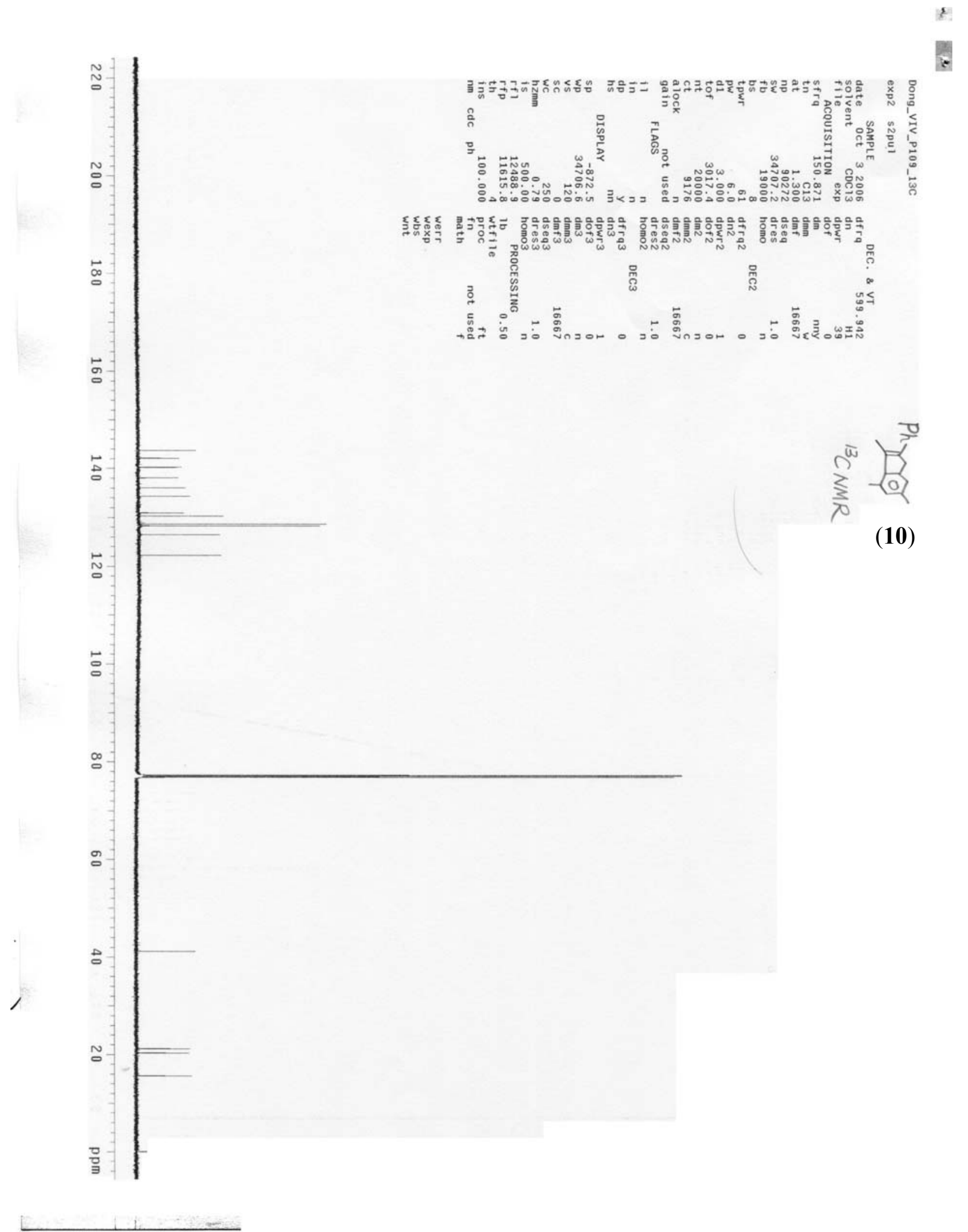

S- 27 

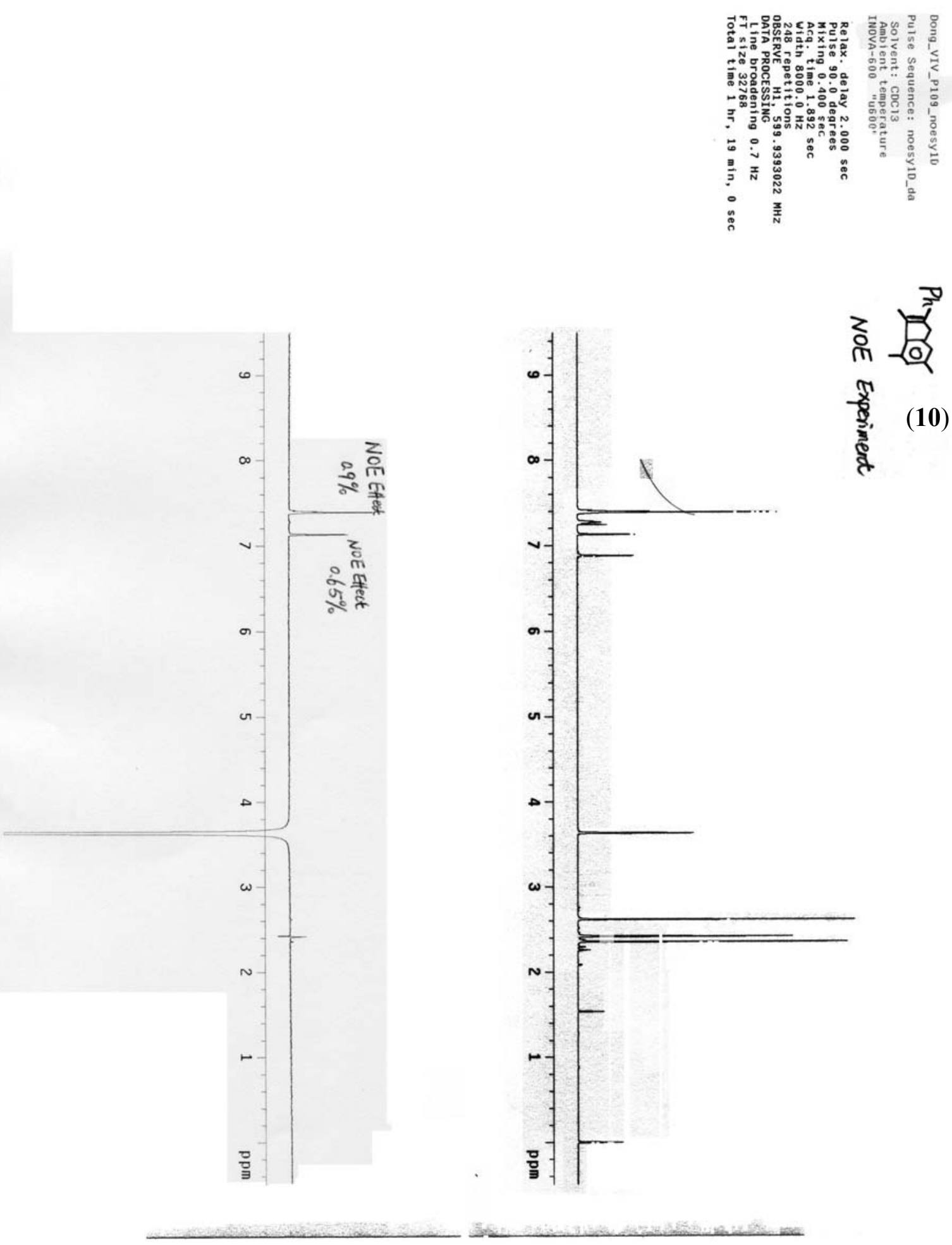

S- 28 


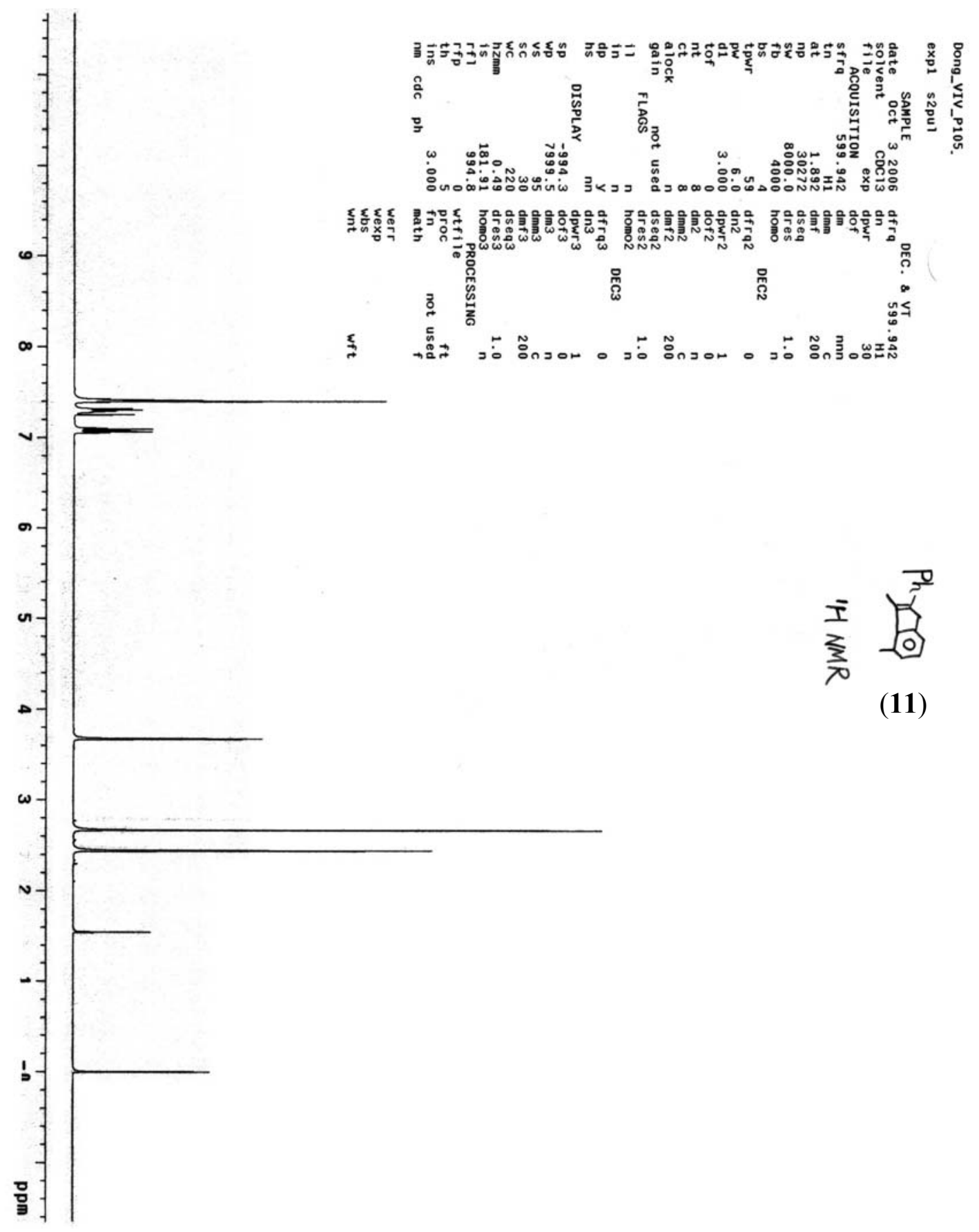

S- 29 

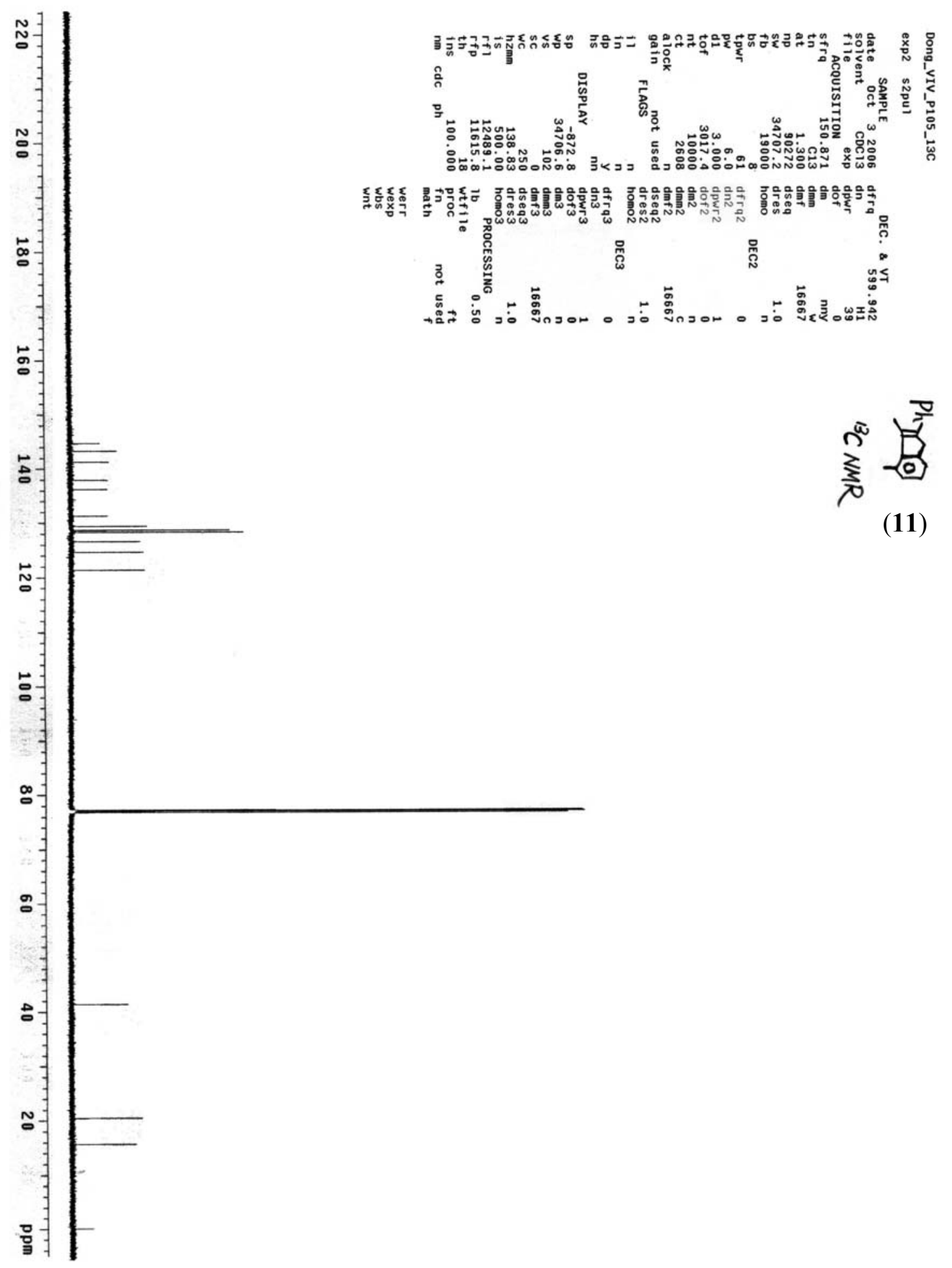

(11) 

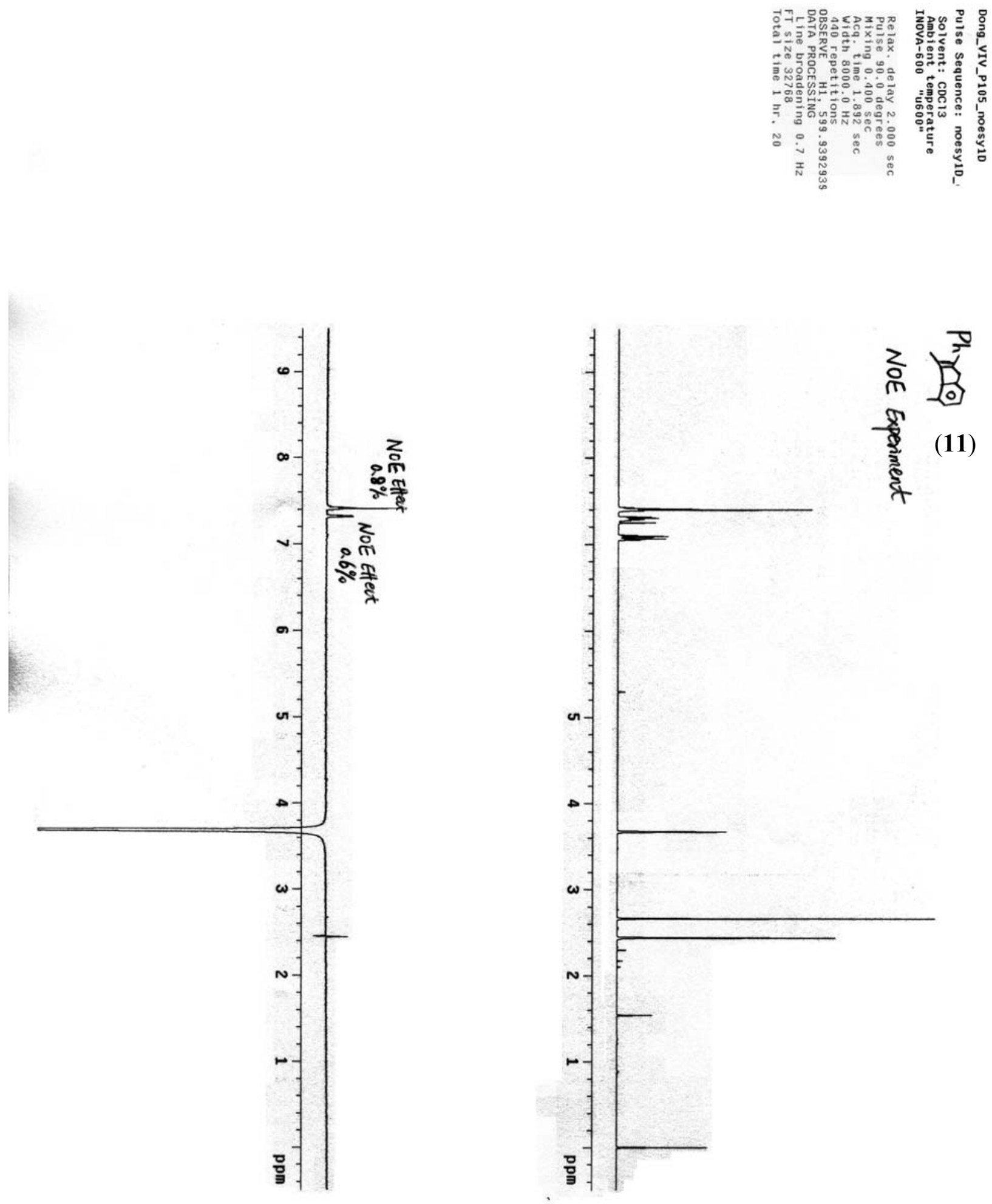


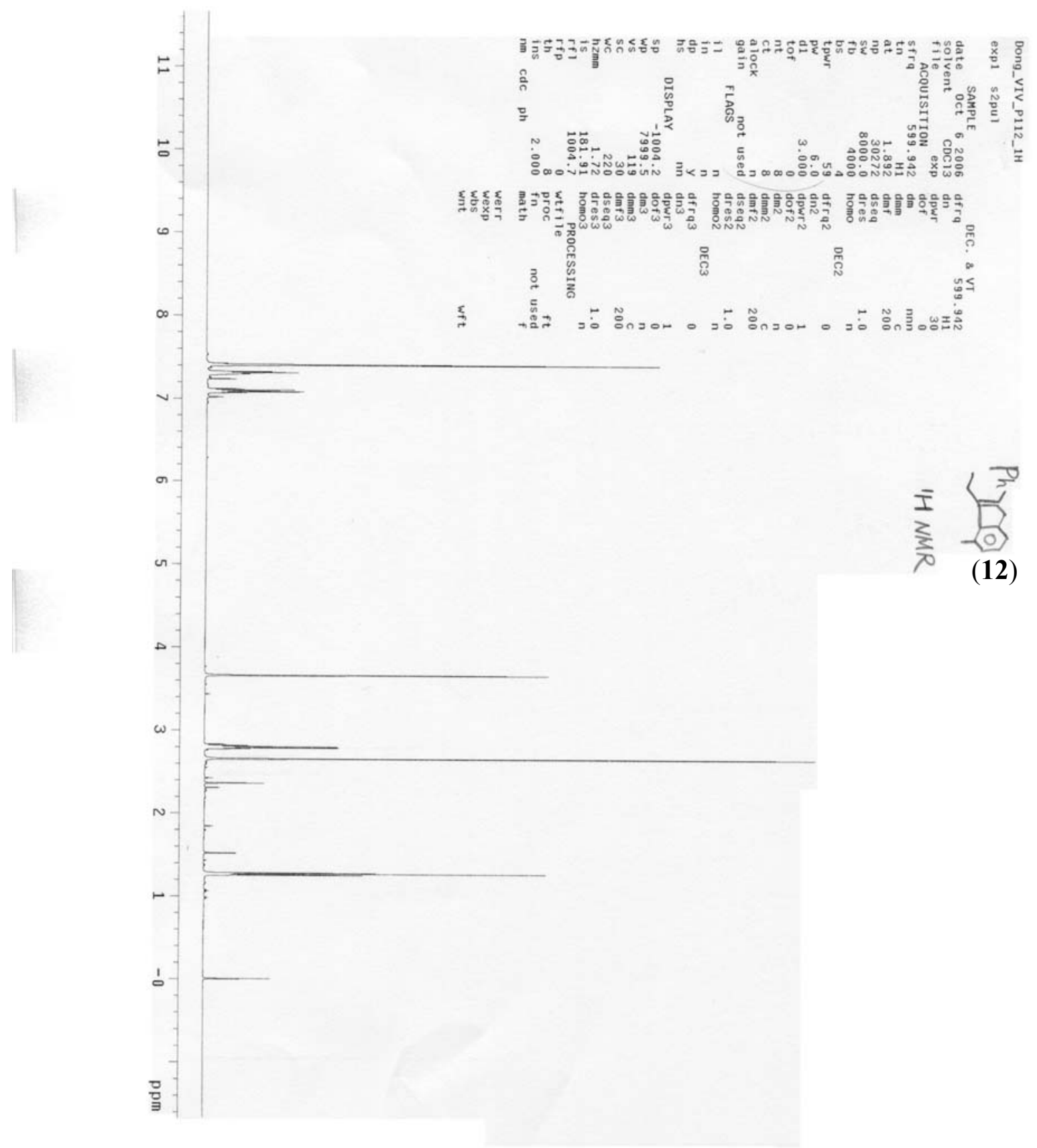

S- 32 


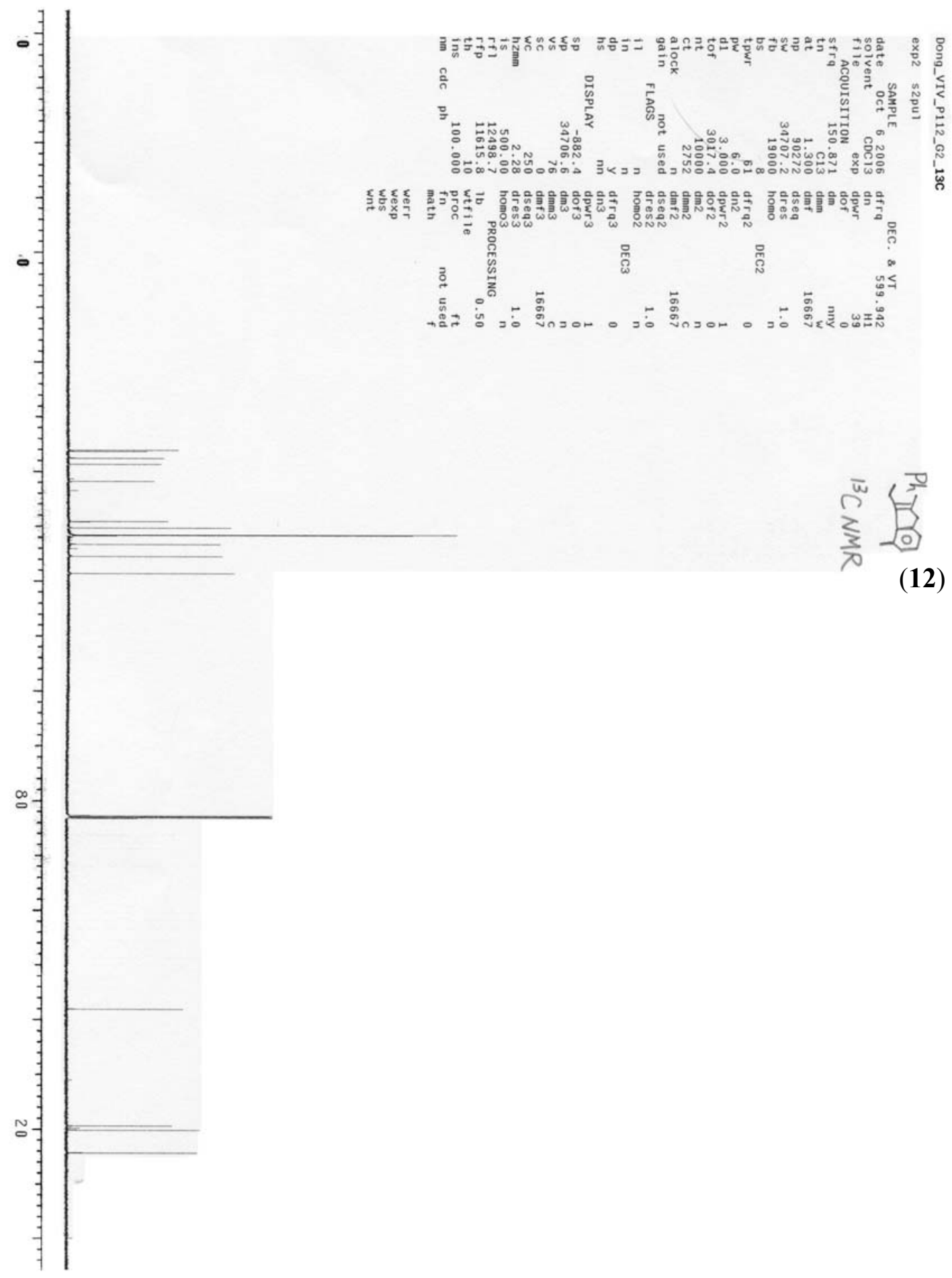




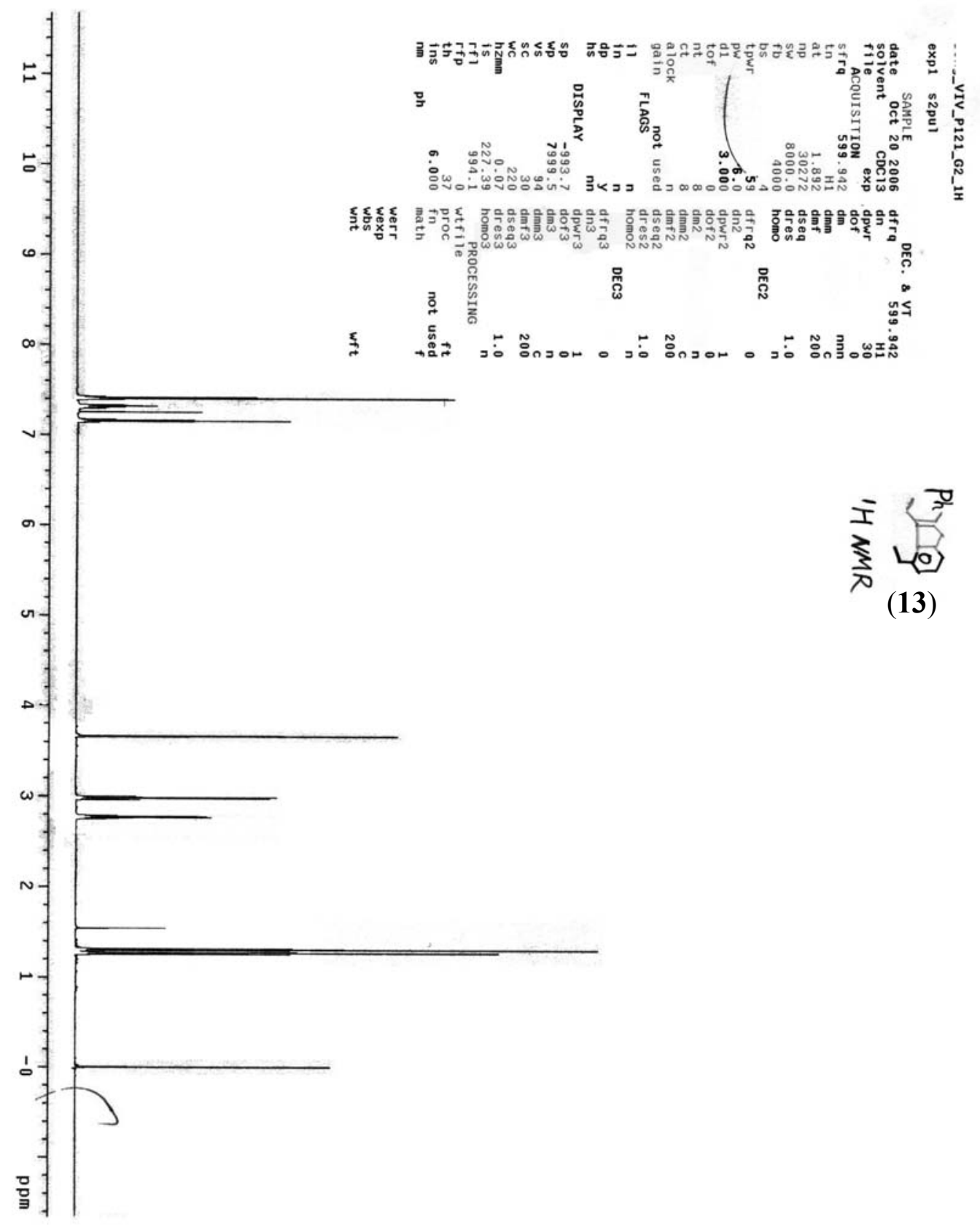




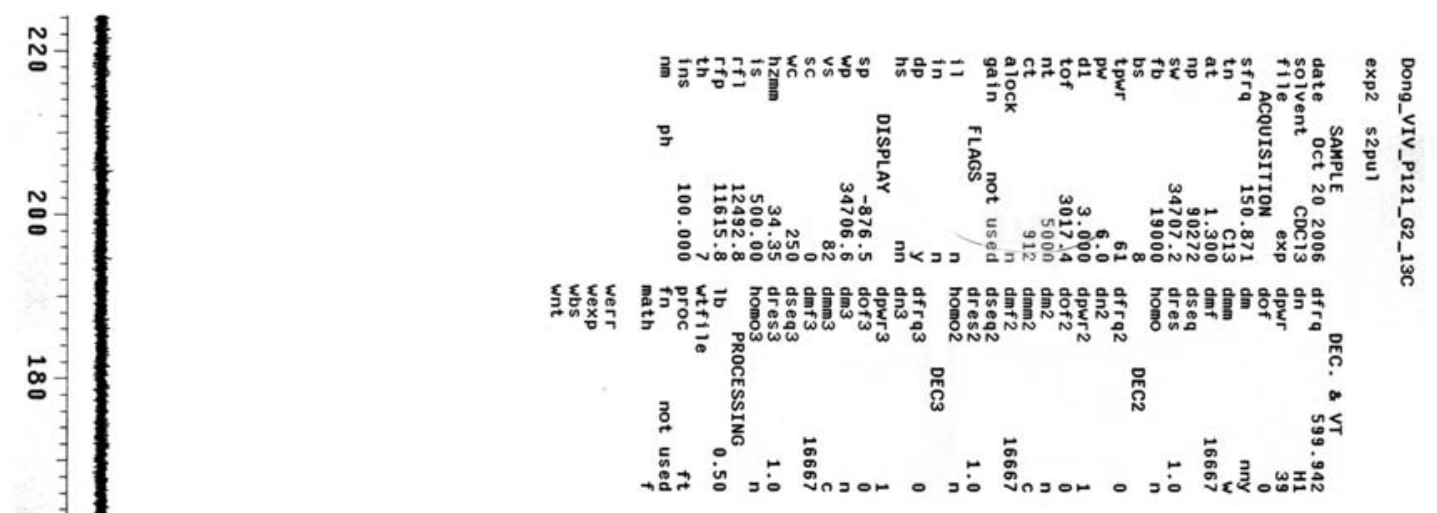

。

品

$\sum_{i}^{\infty}$

응

栆

g

a

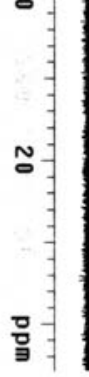

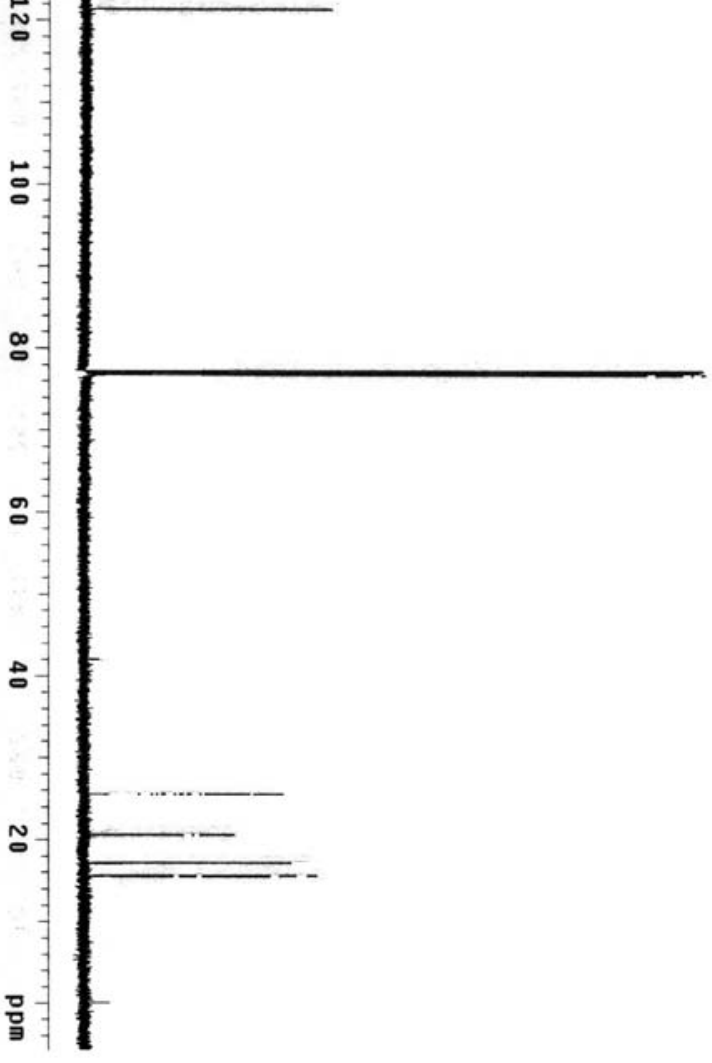



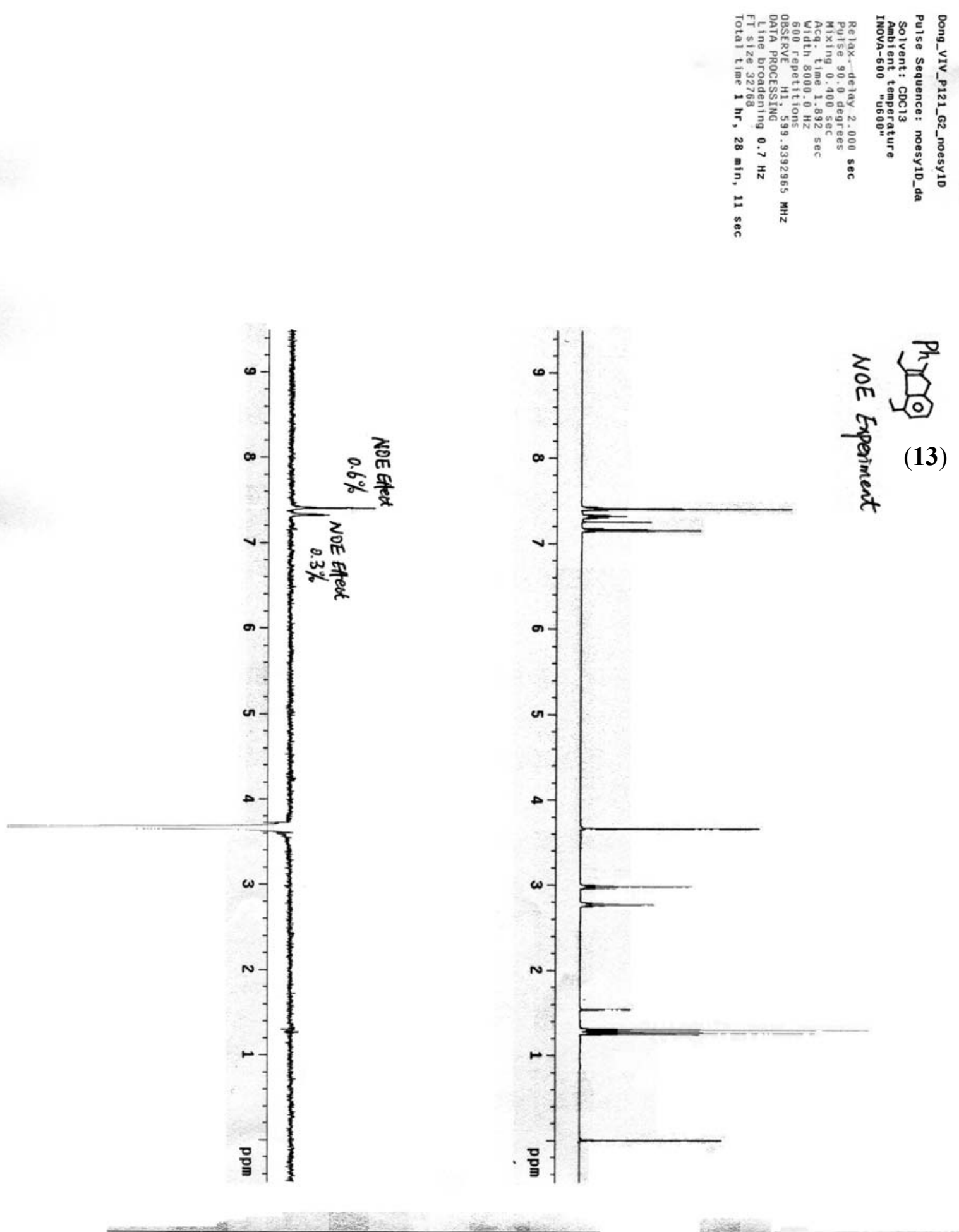

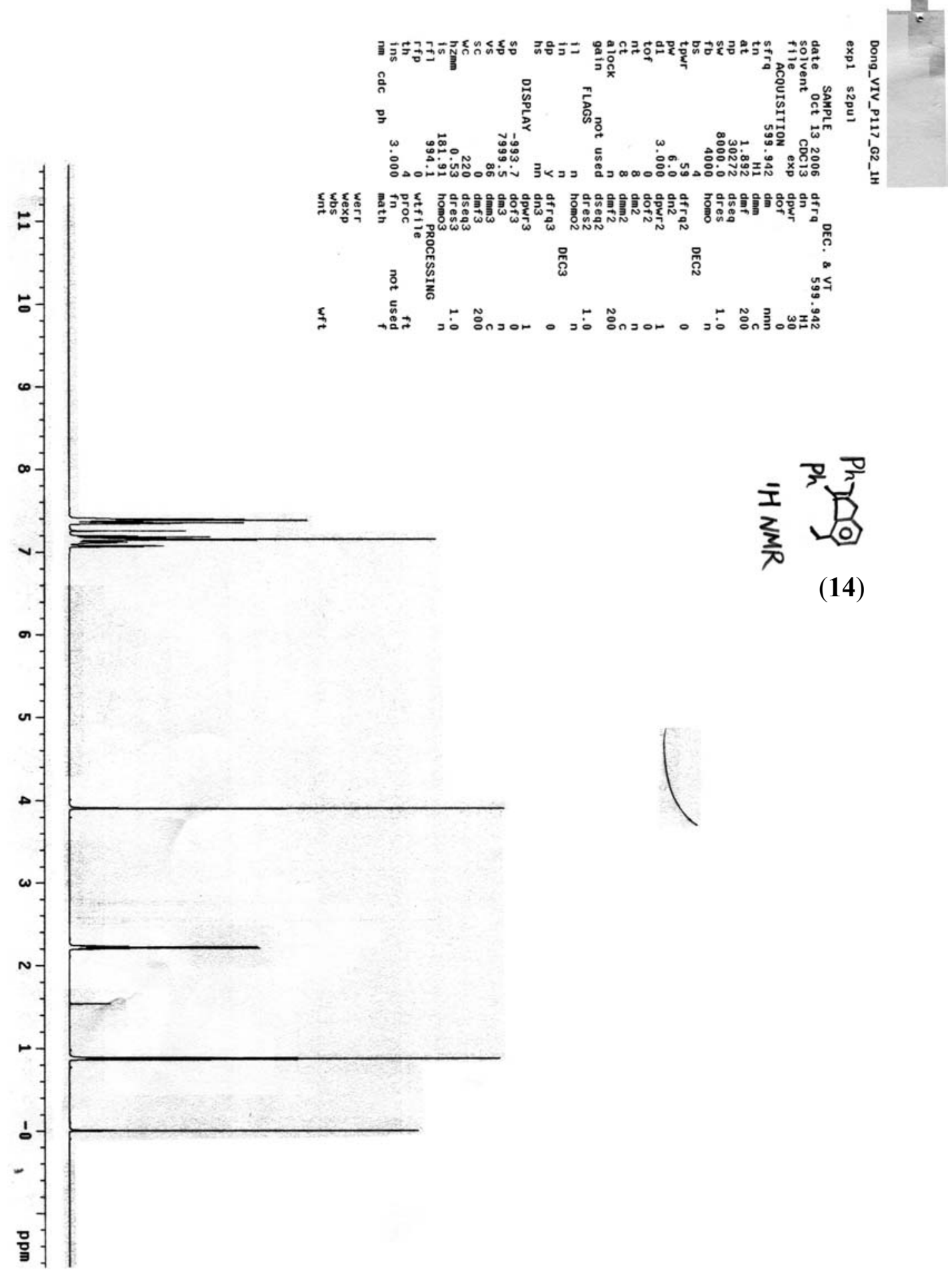

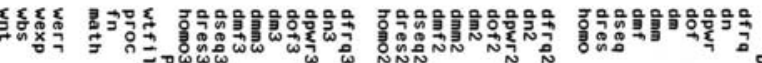

(14) 


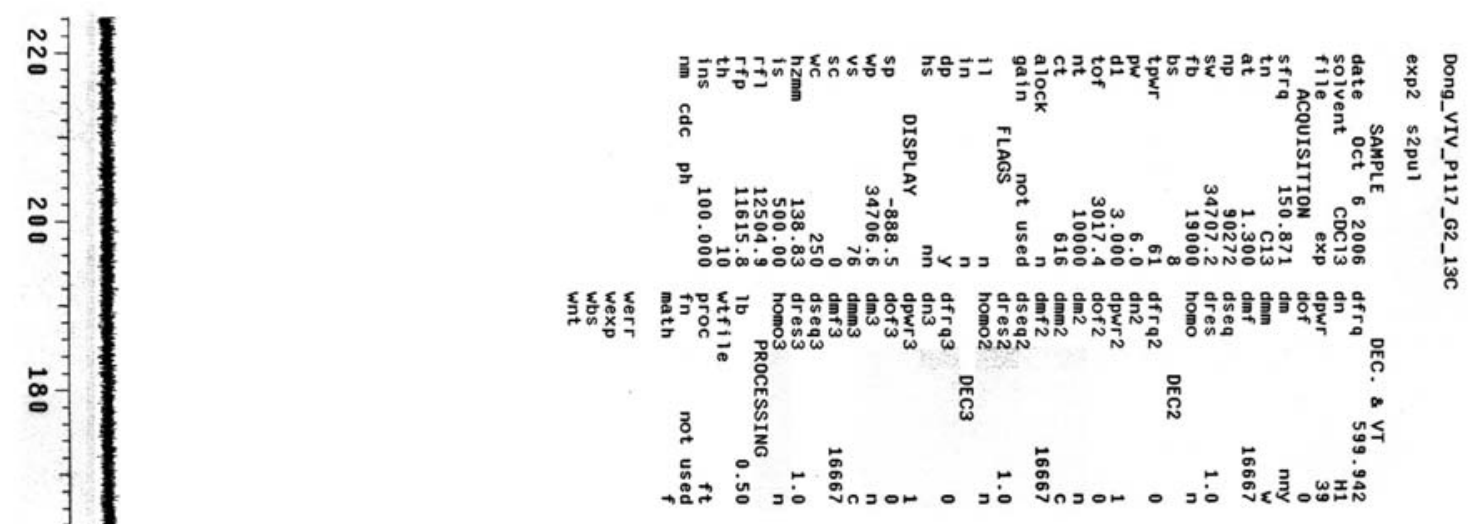

吕

$\stackrel{\circ}{\circ}$
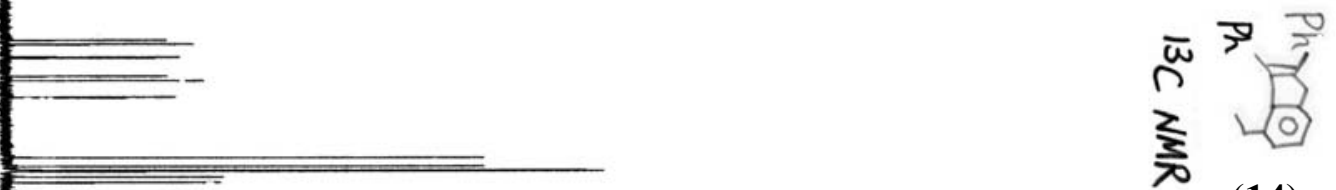

s.

농

$\stackrel{\infty}{\circ}$

a

$\therefore-$

ㄴ-

뭌

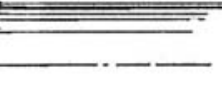




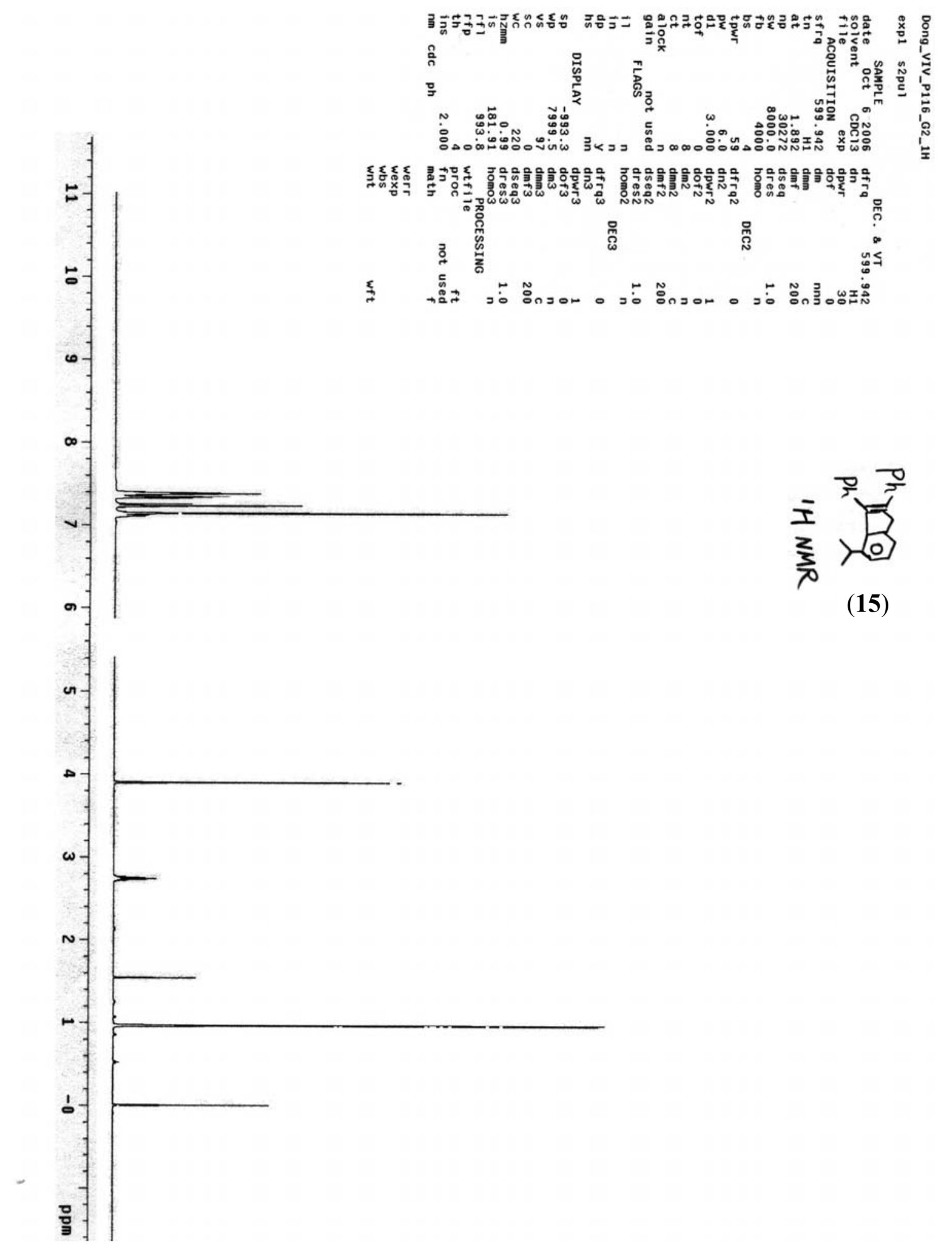




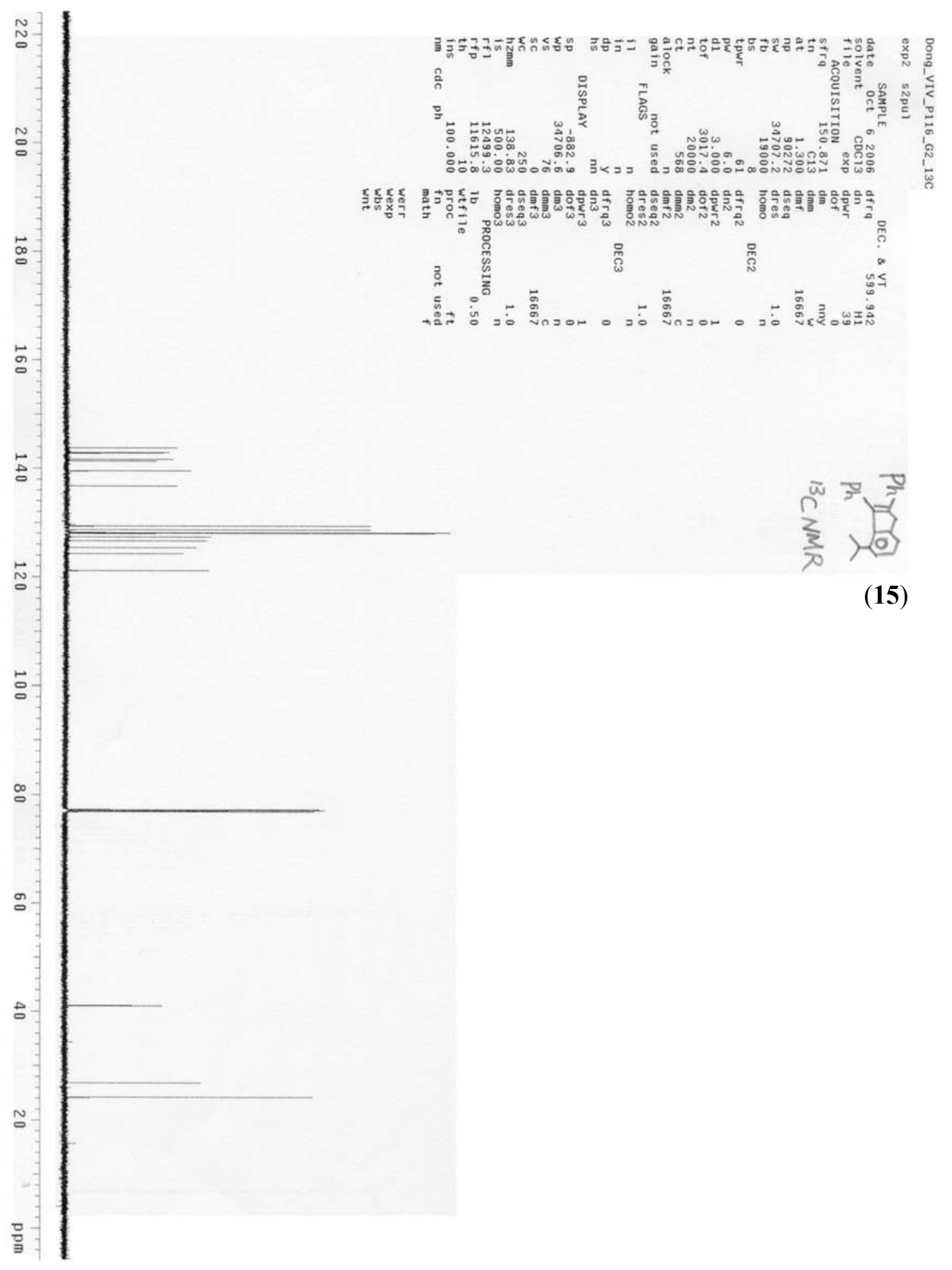




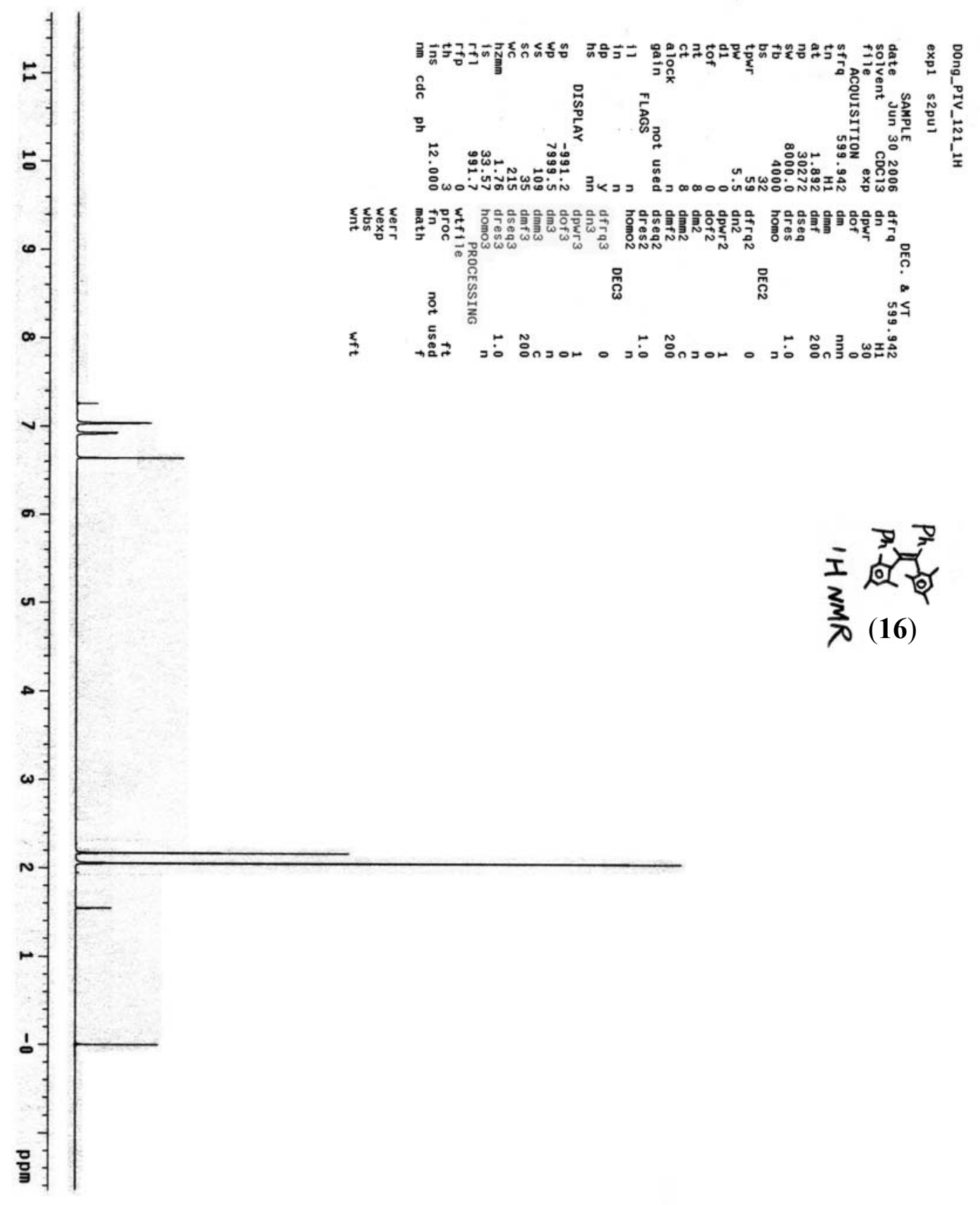

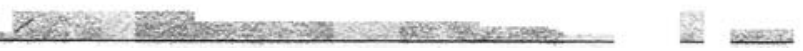



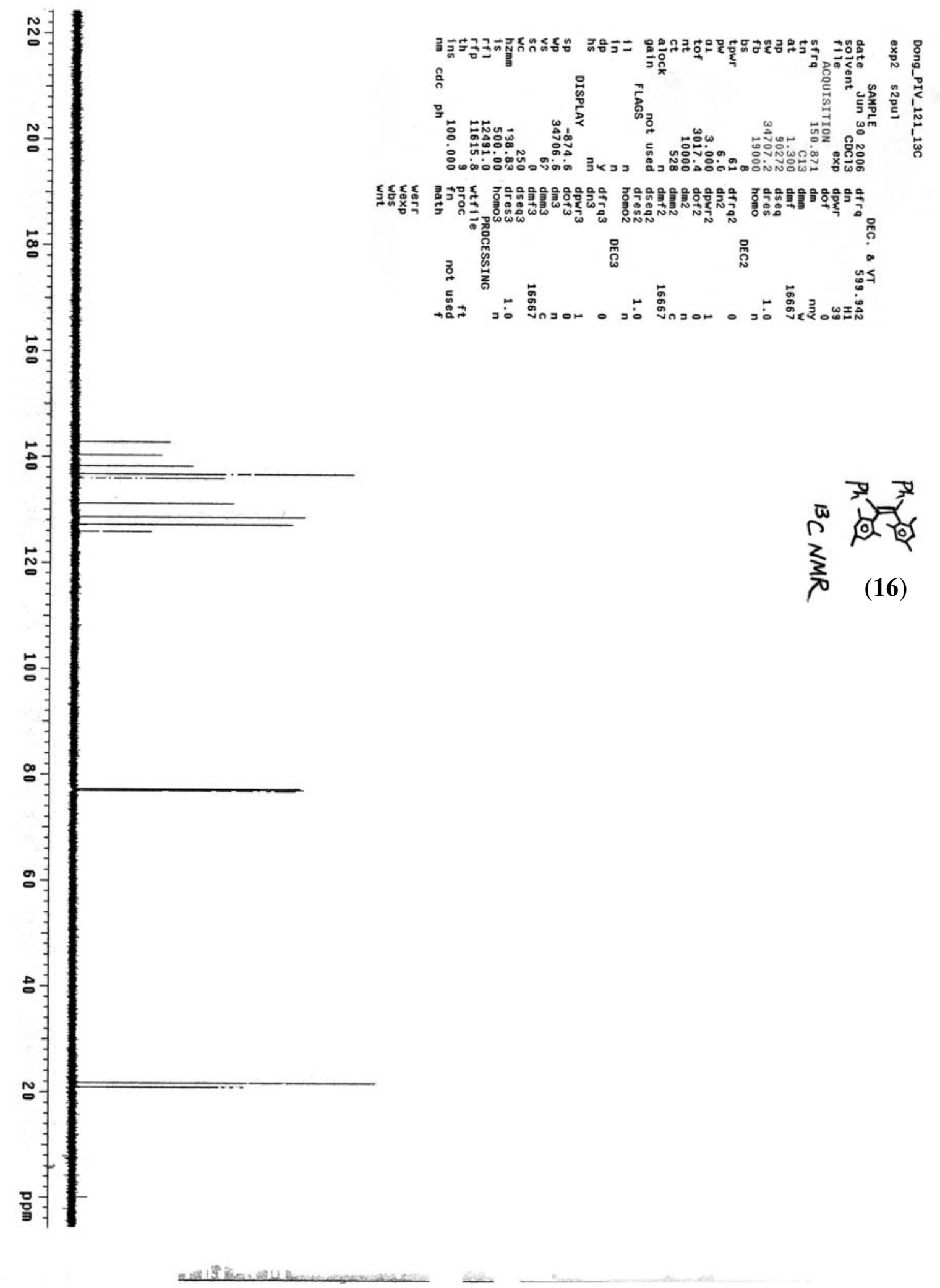

S- 42 


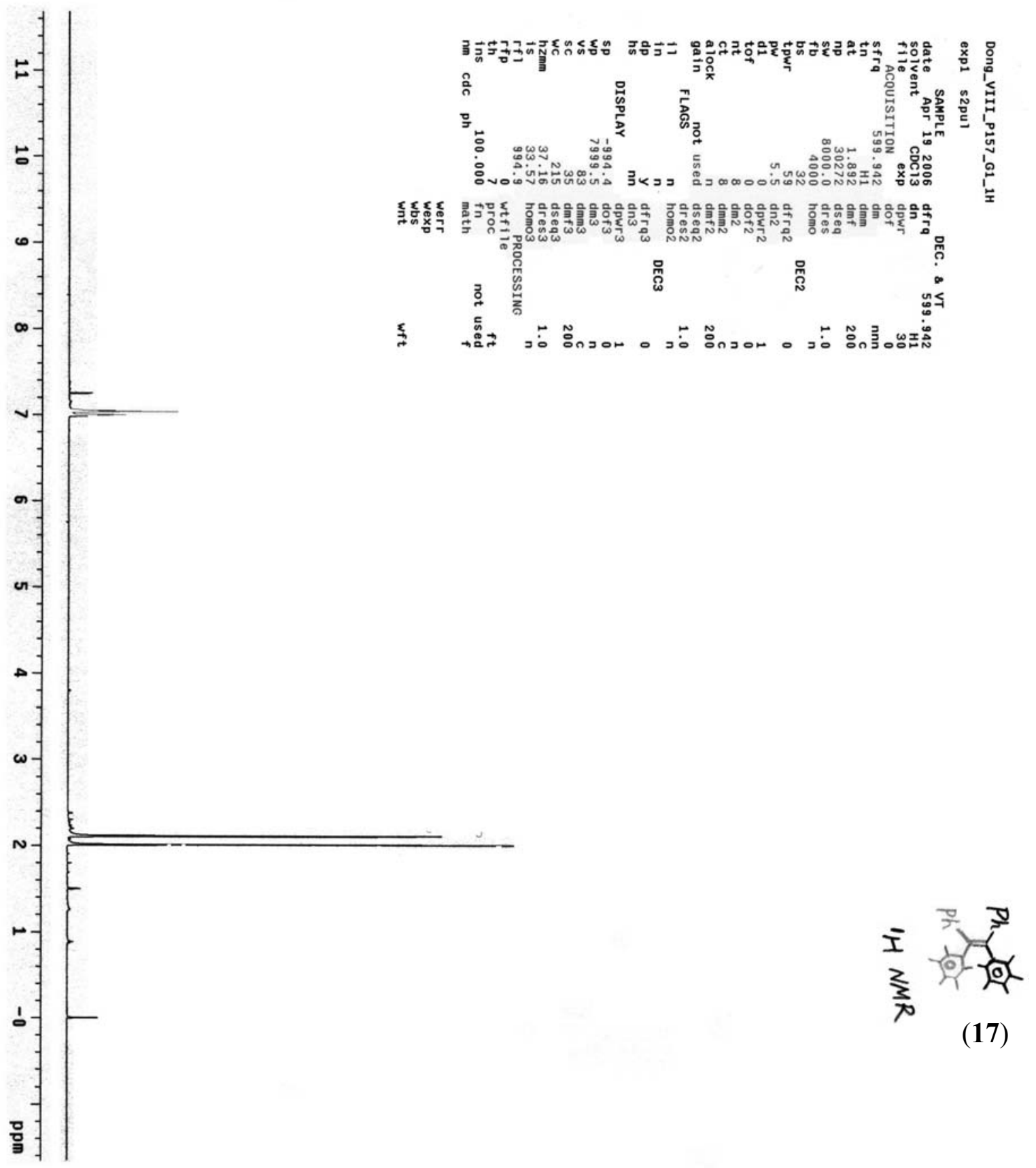



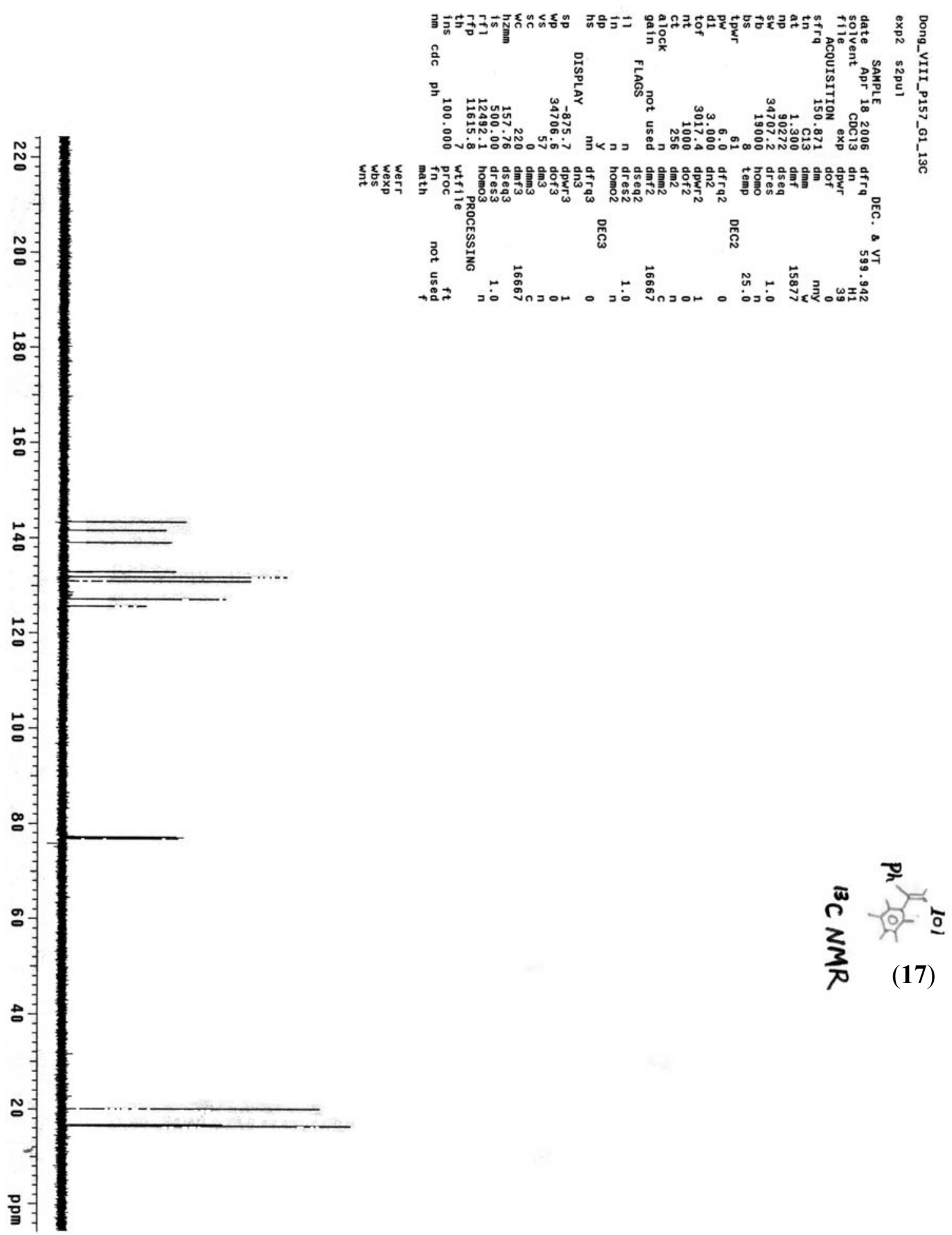


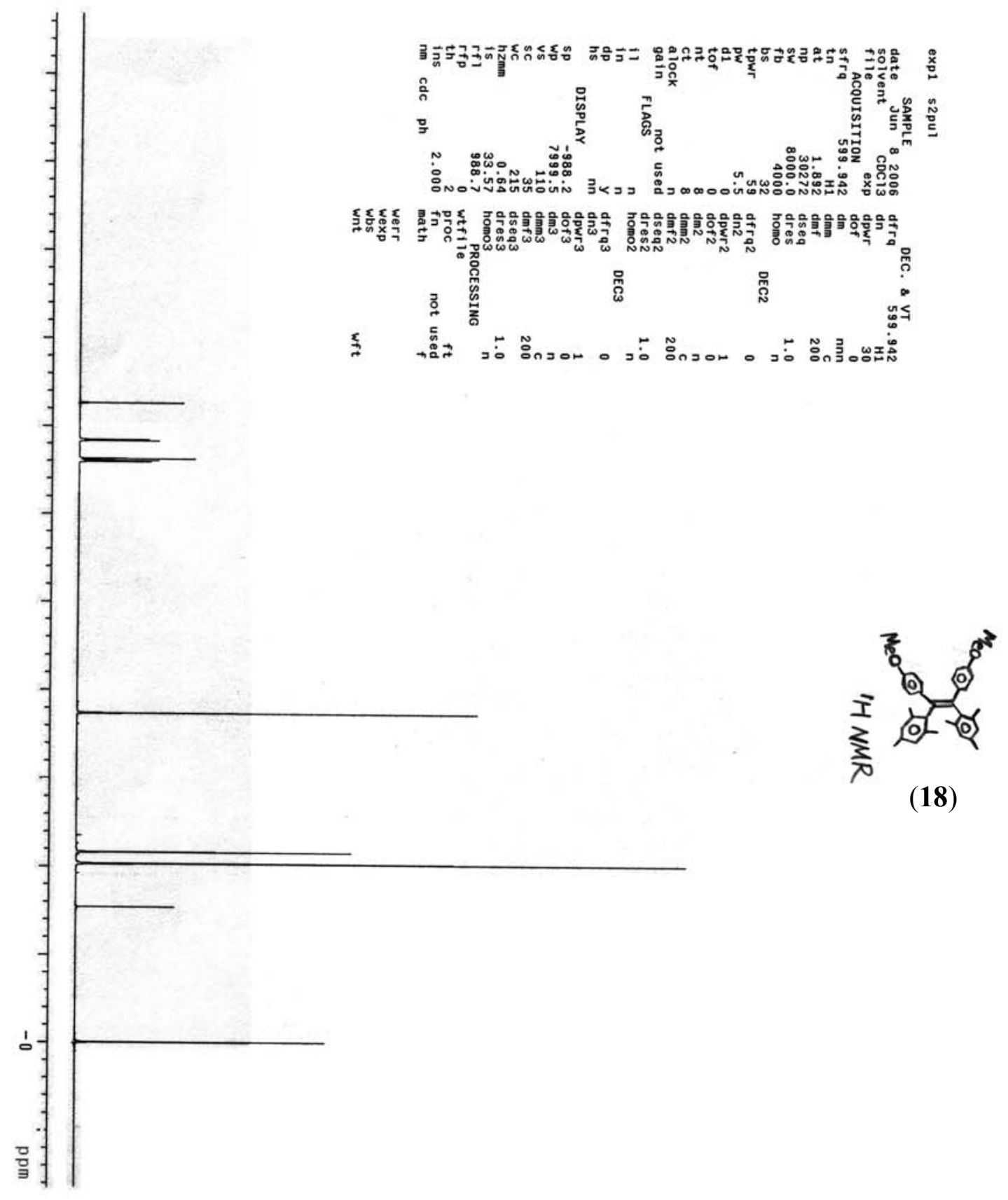




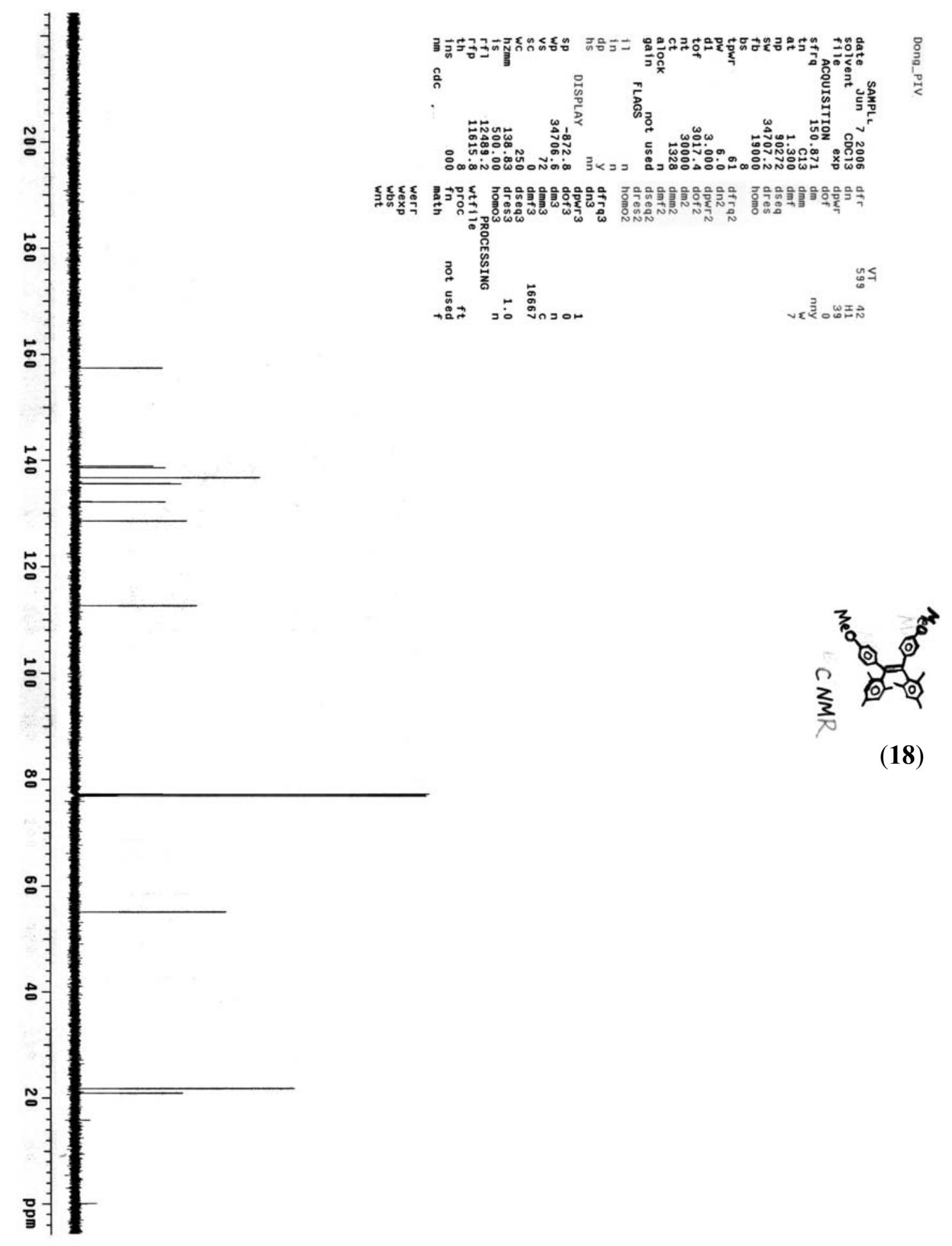




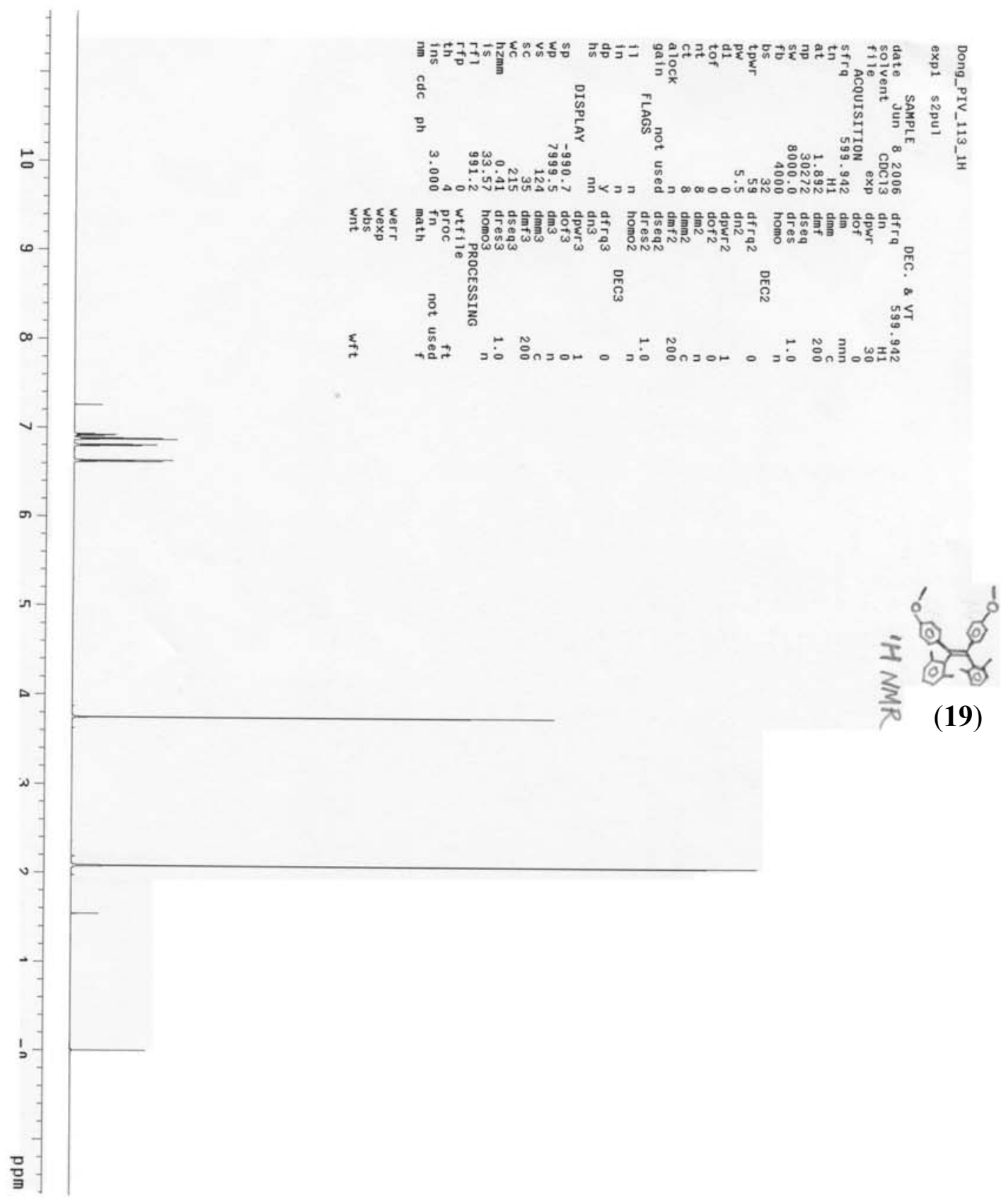



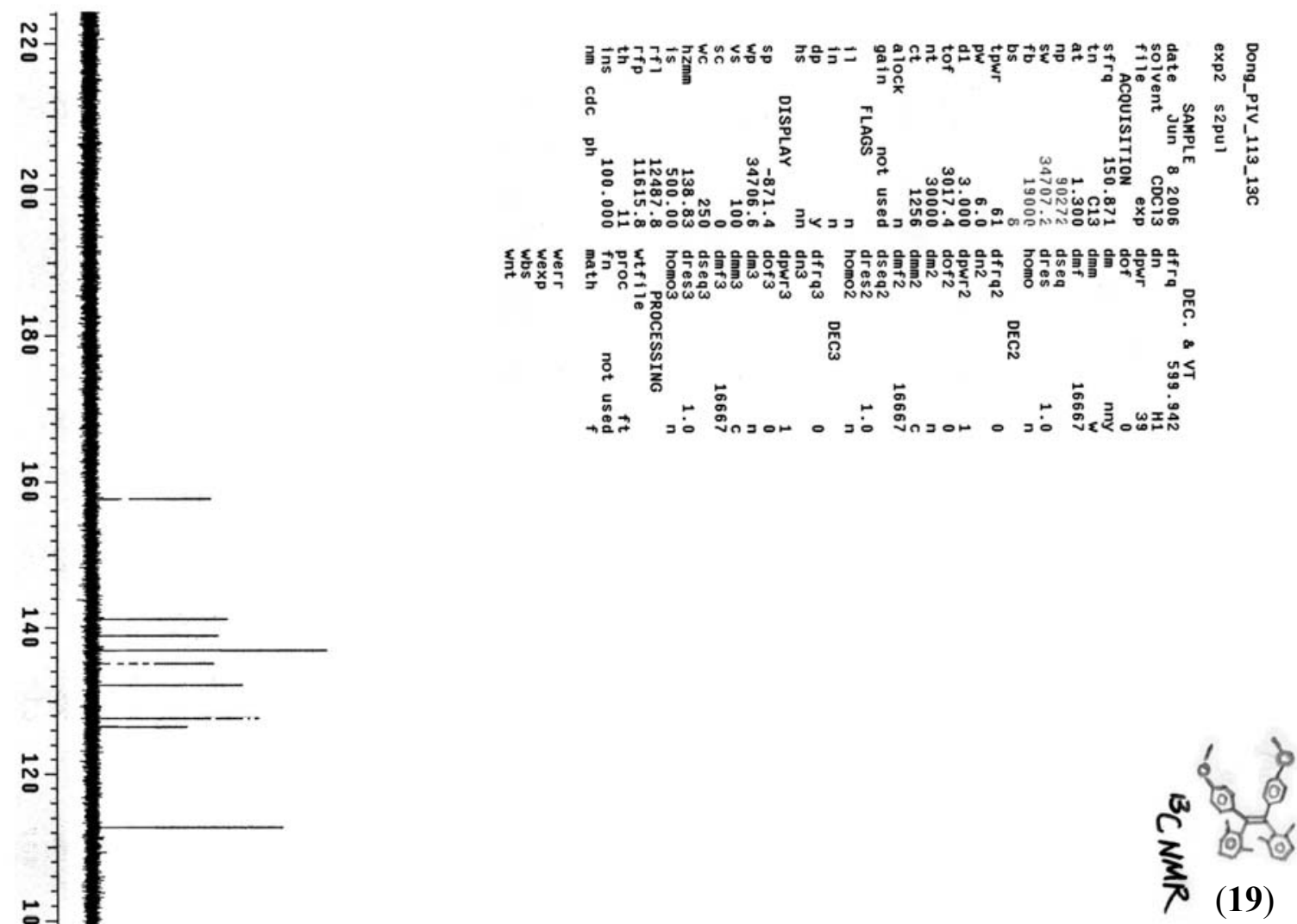


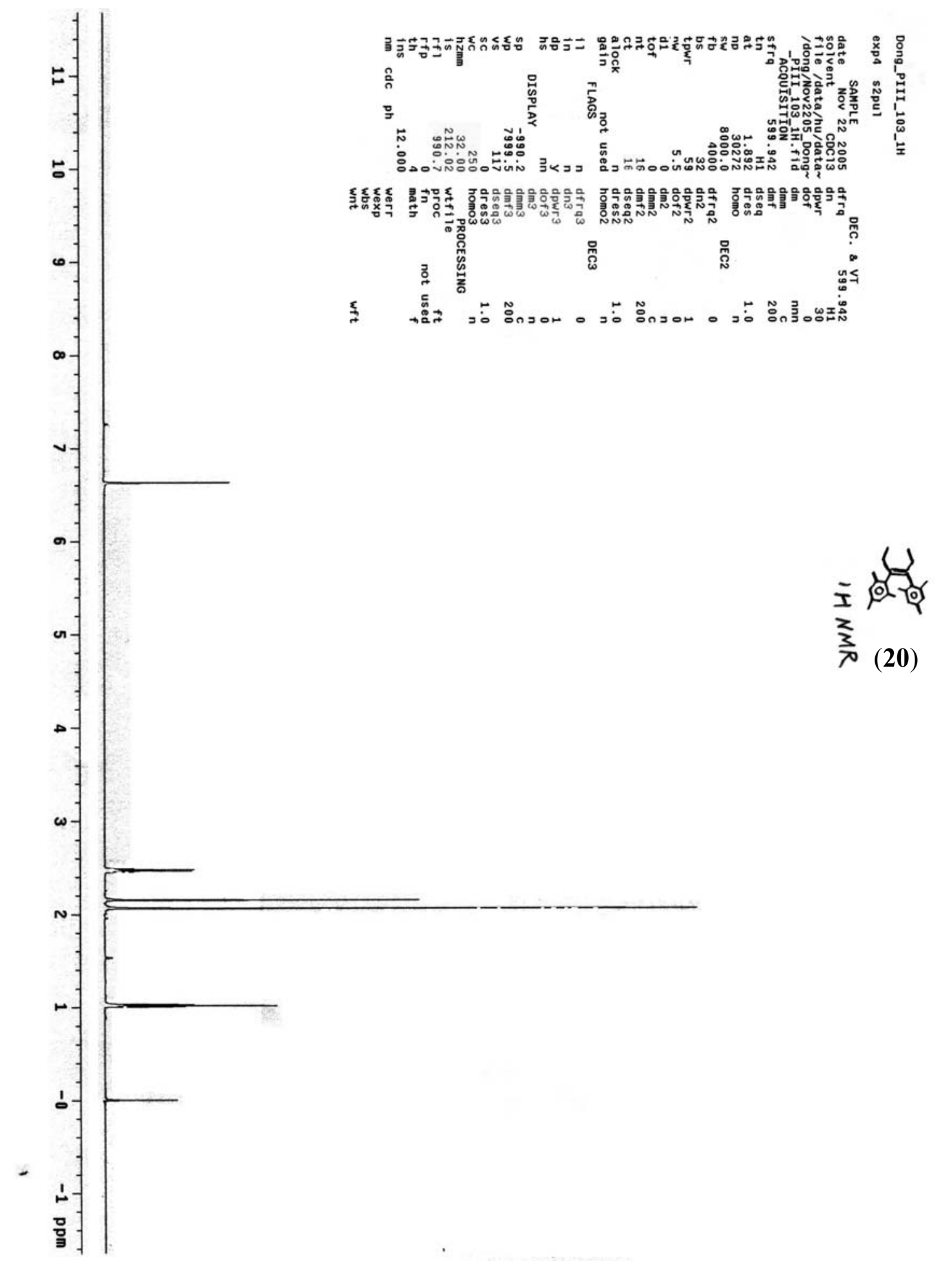




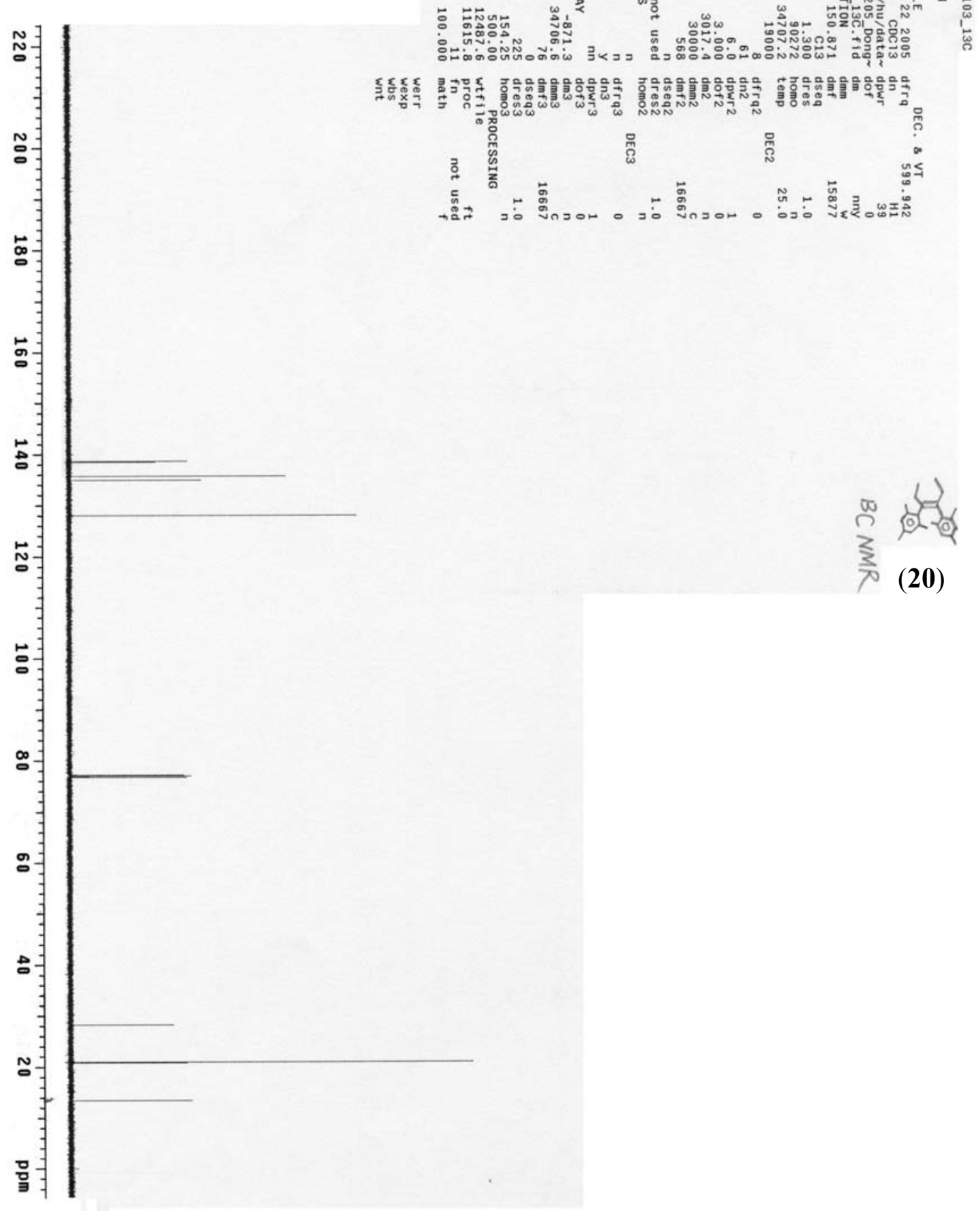




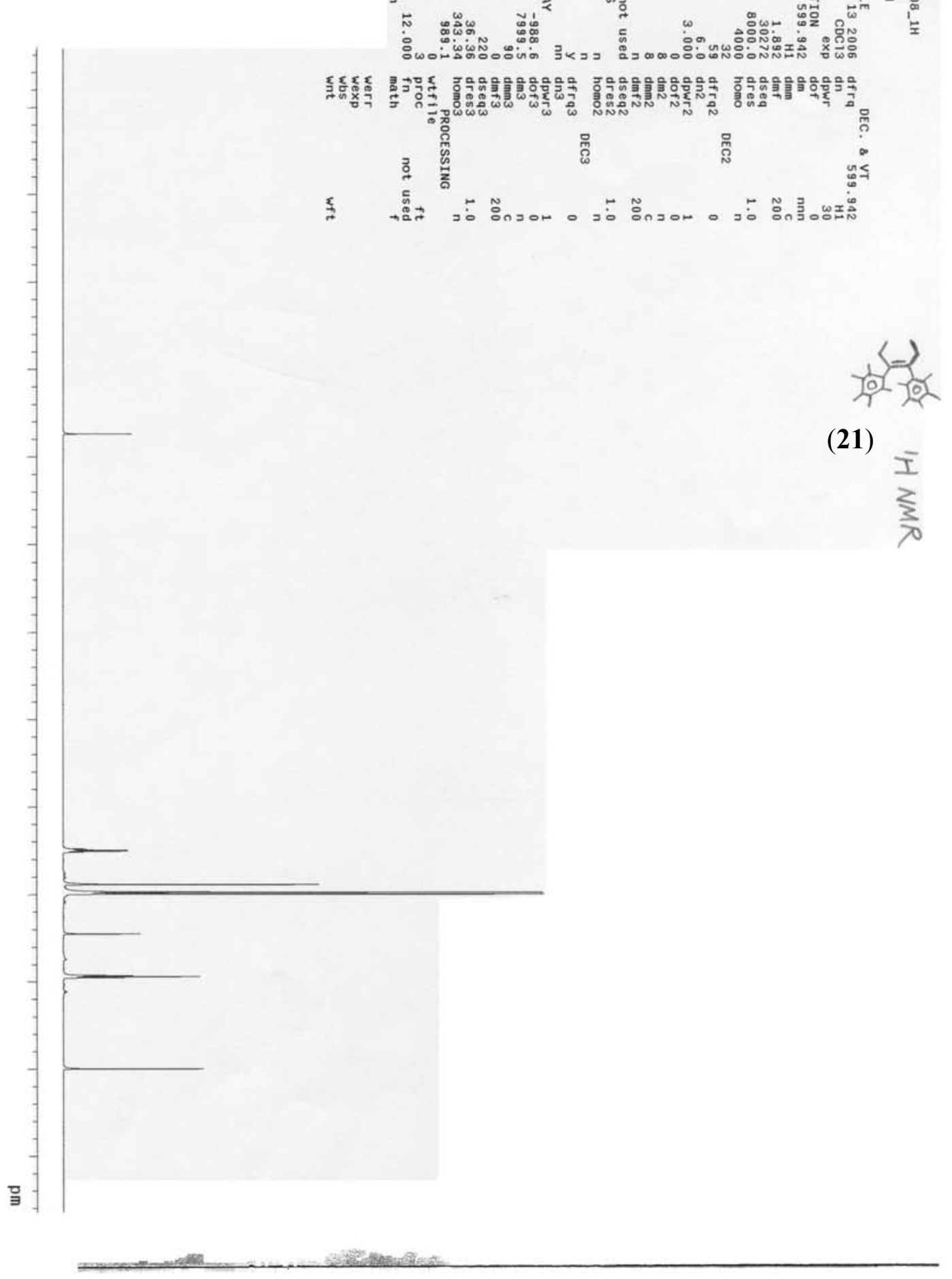

S- 51 


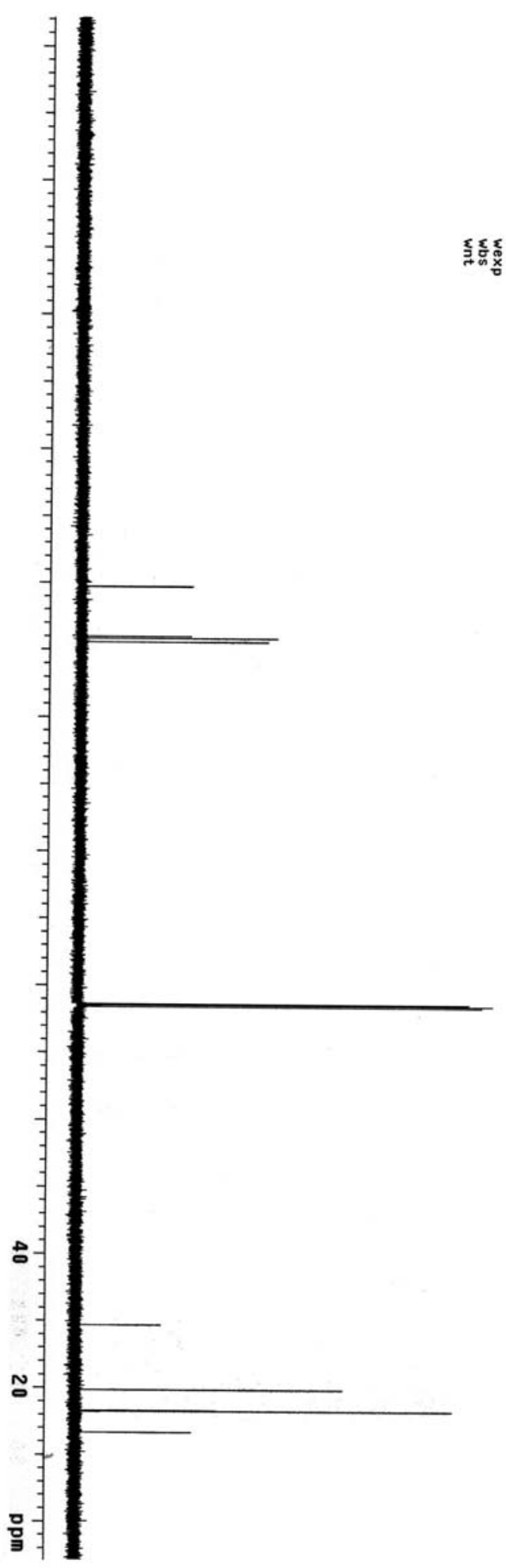

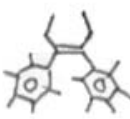

(21)

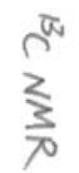




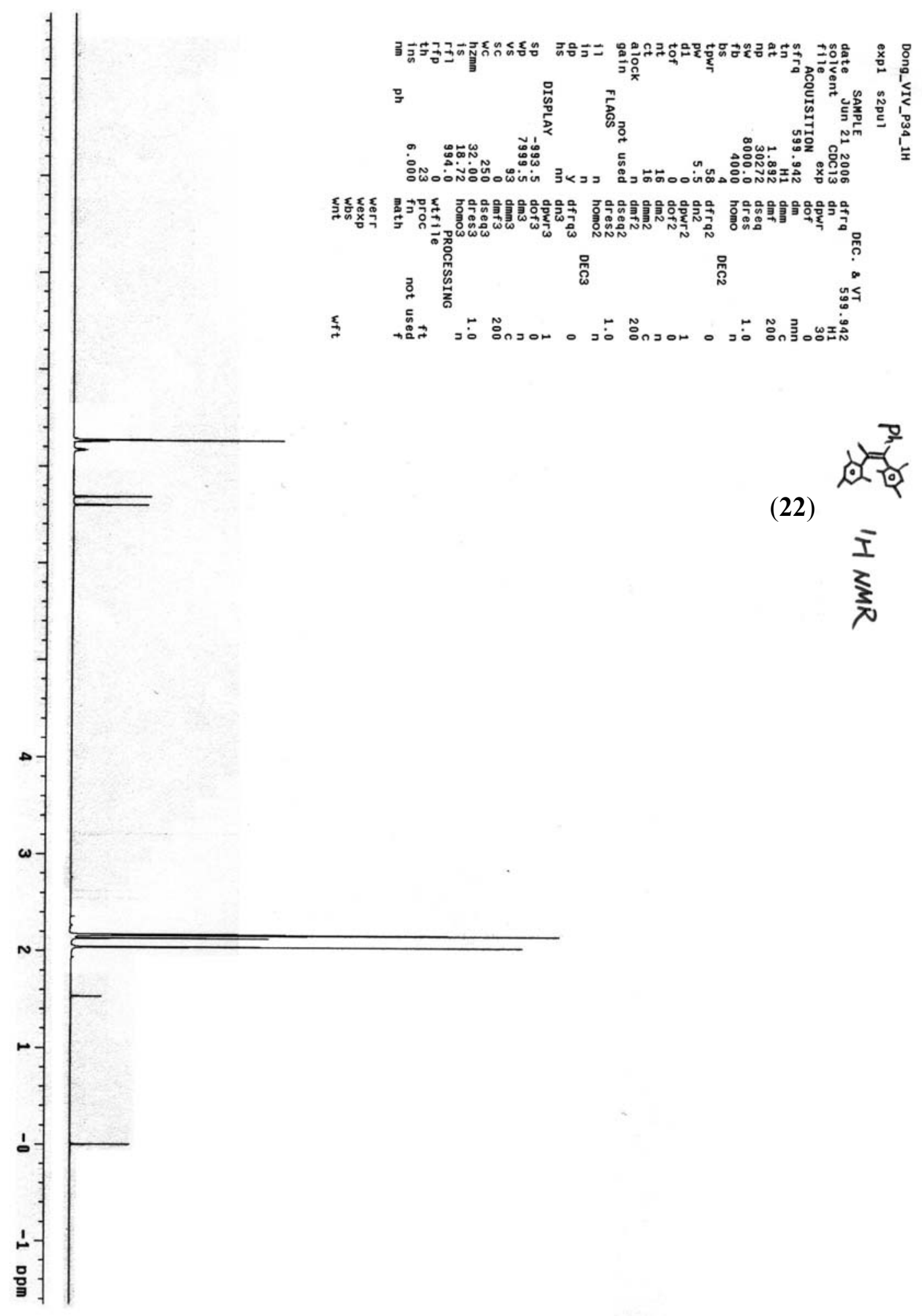

S- 53 


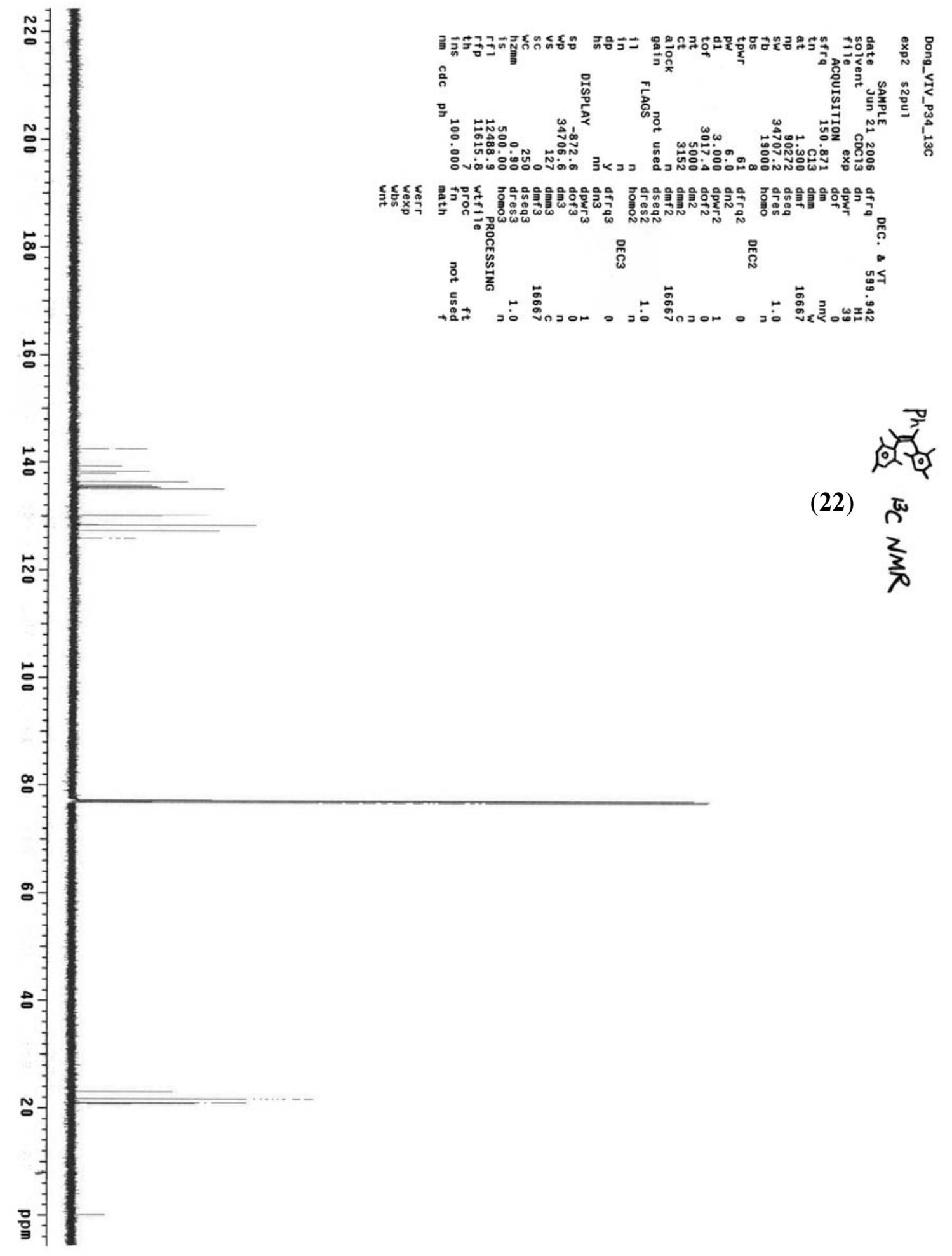

S- 54 


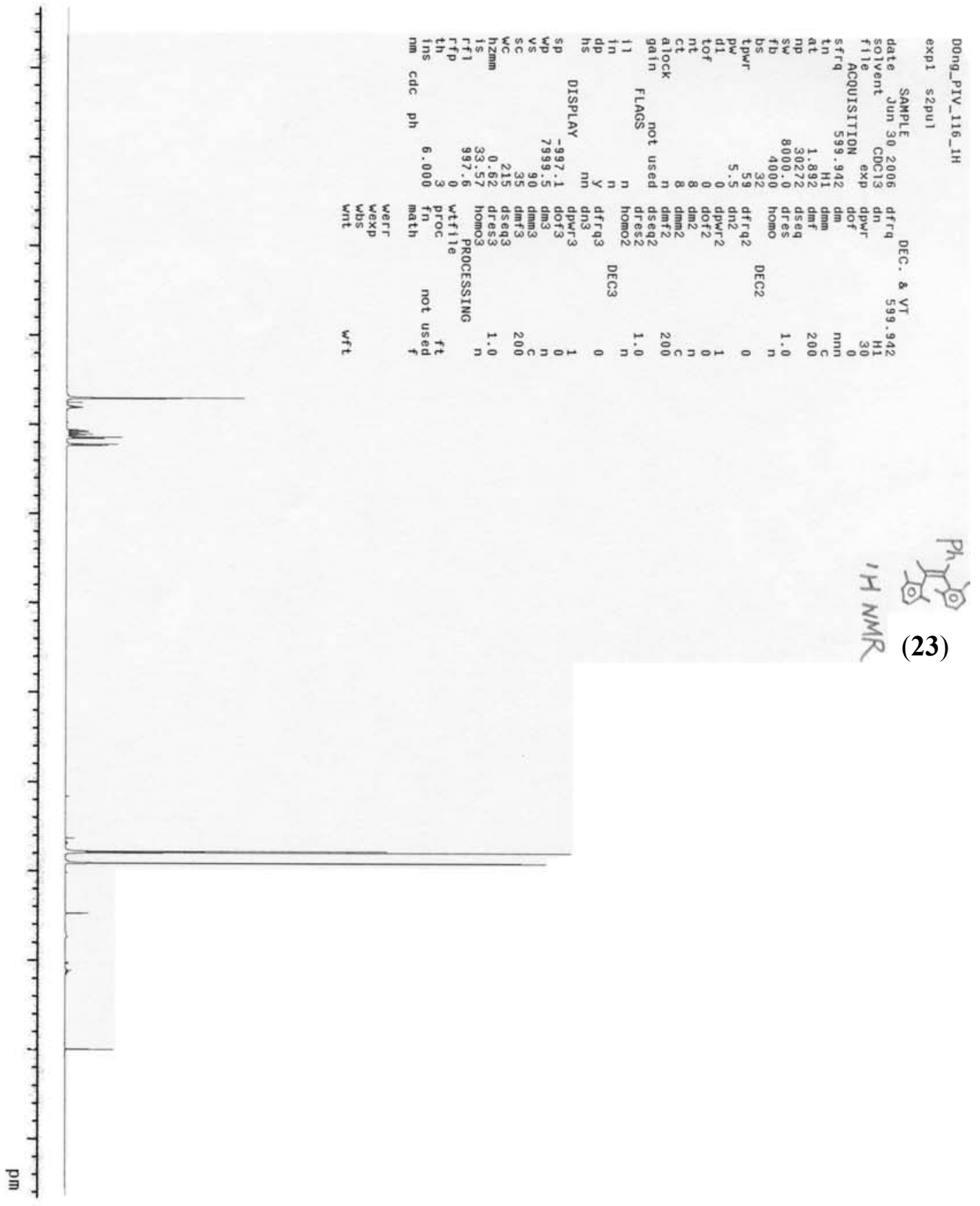

S- 55 


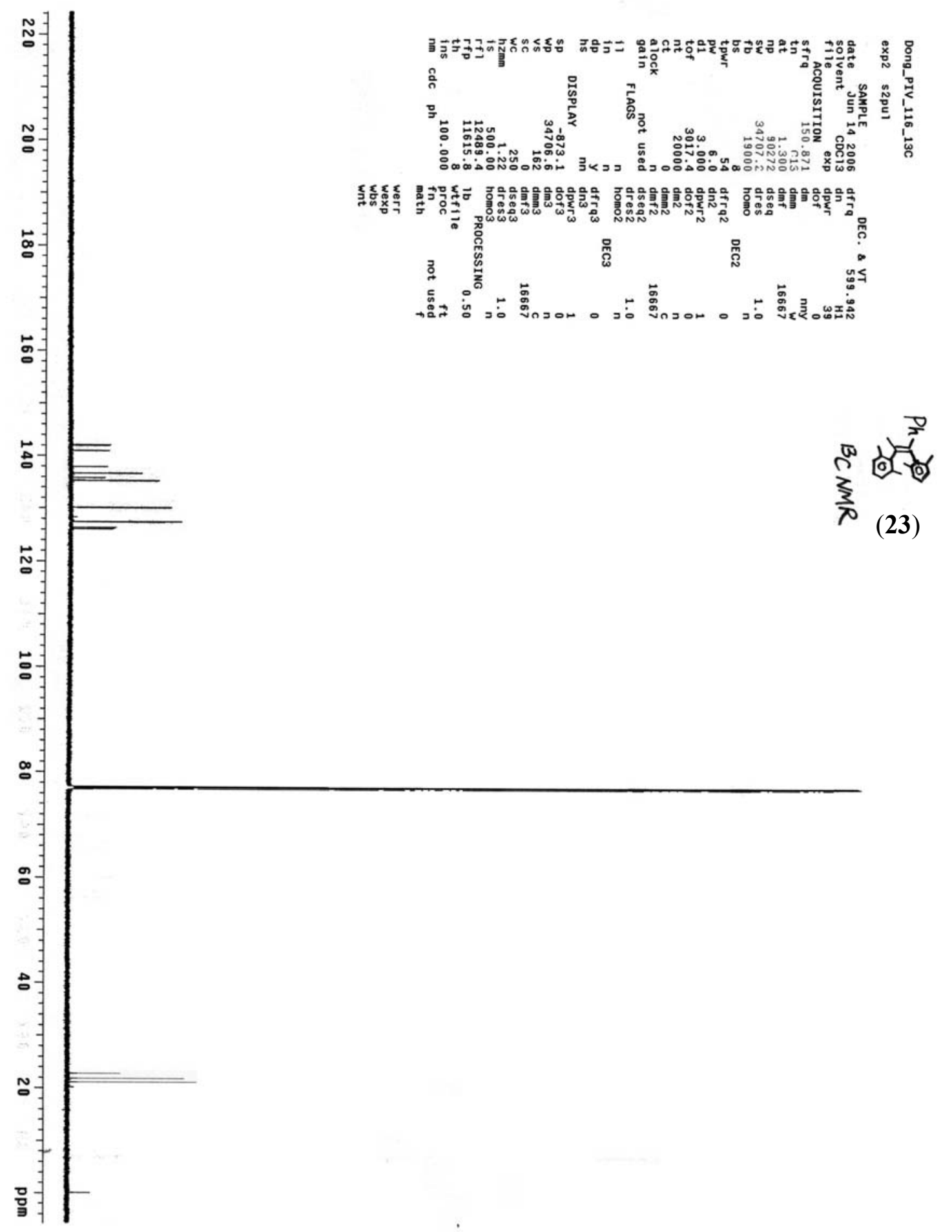

S- 56 

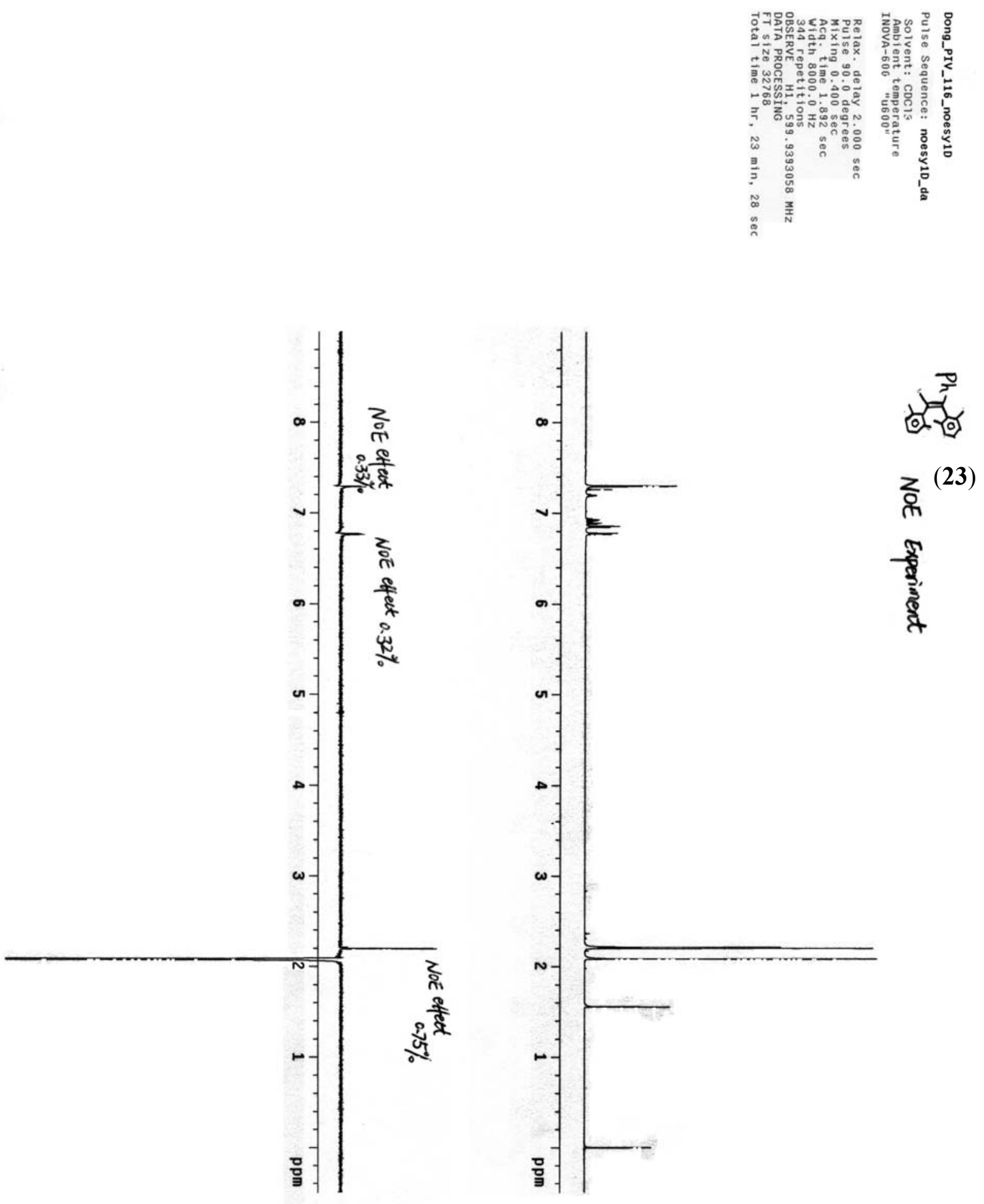


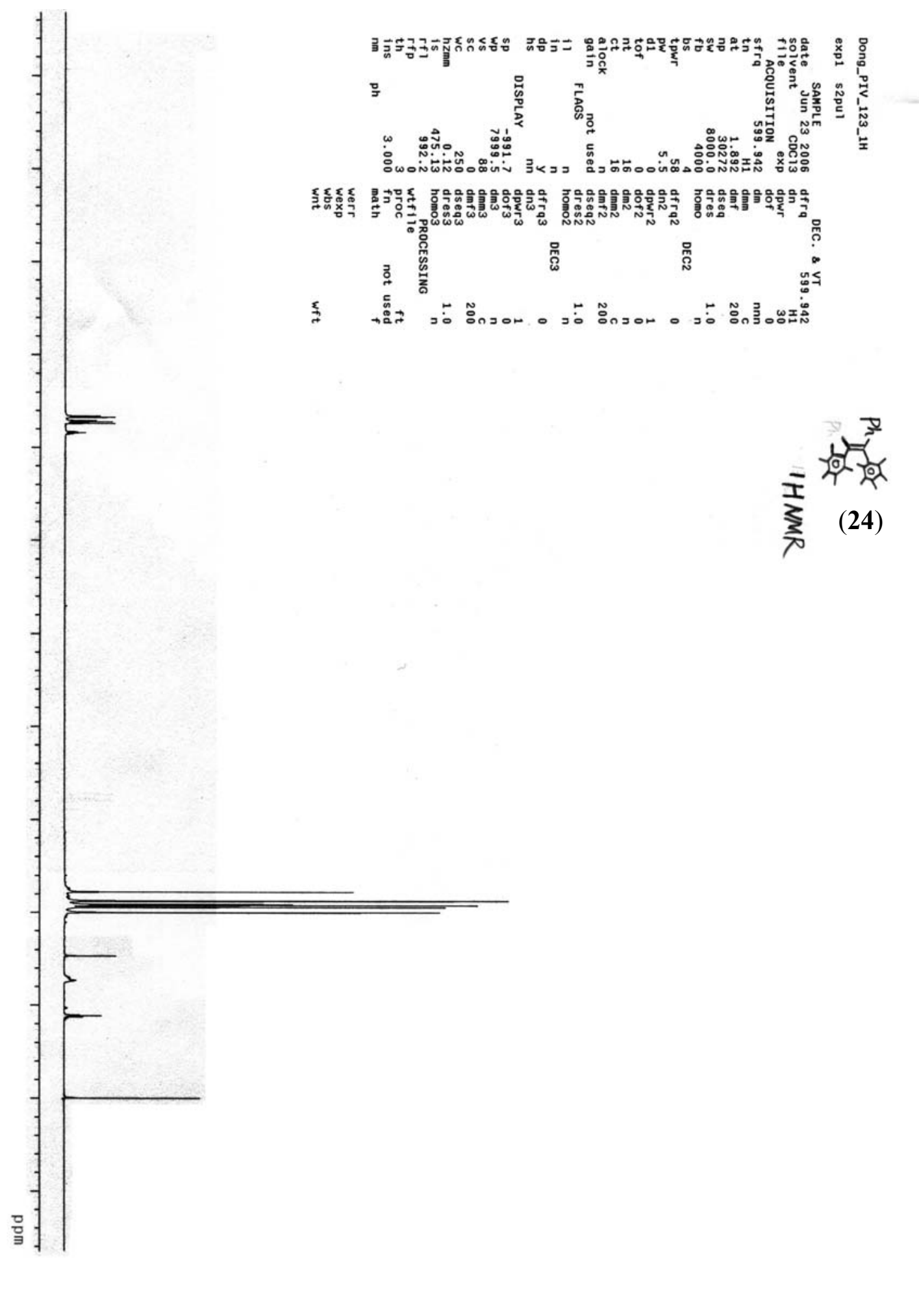

S- 58 

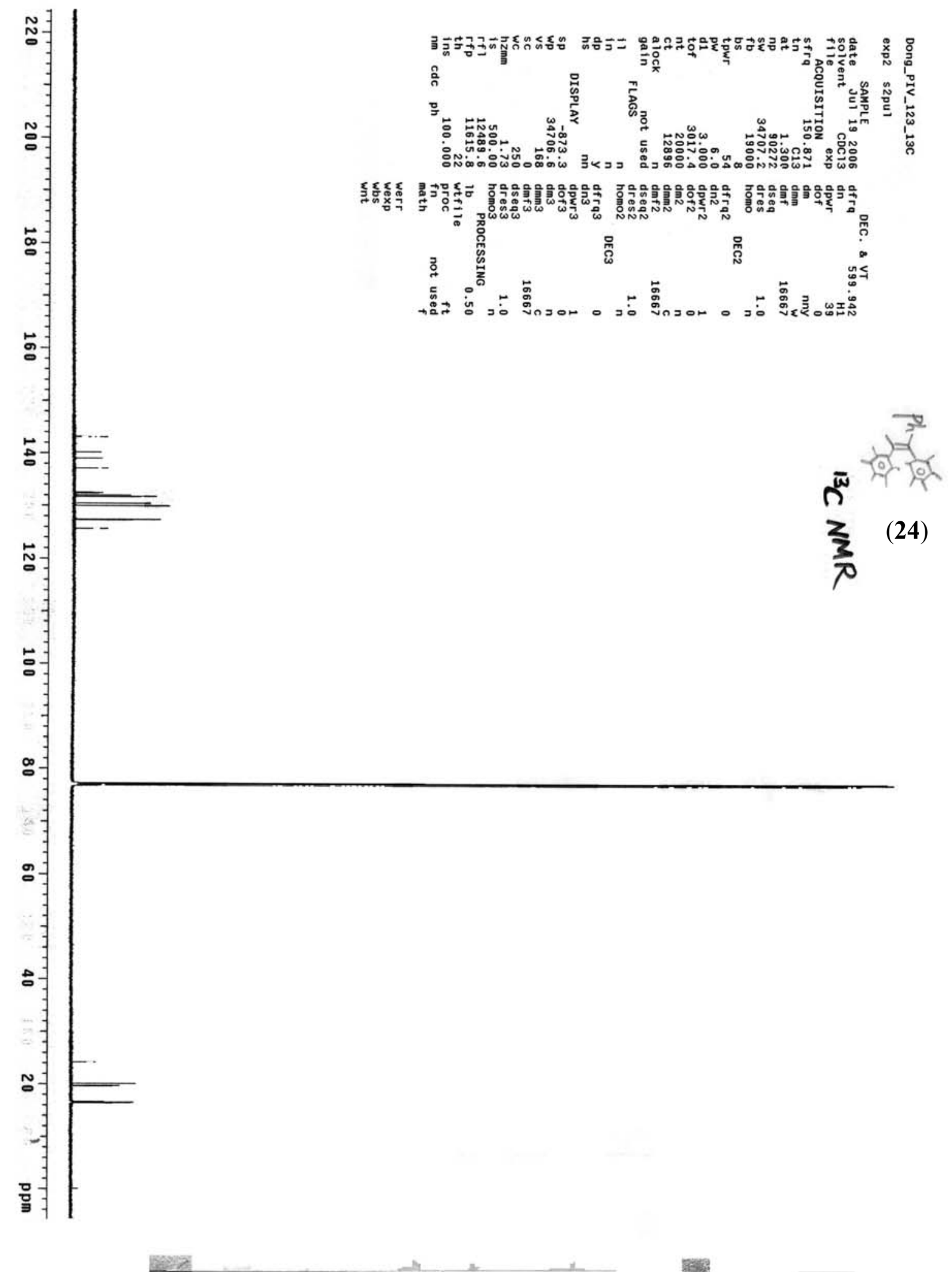

S- 59 

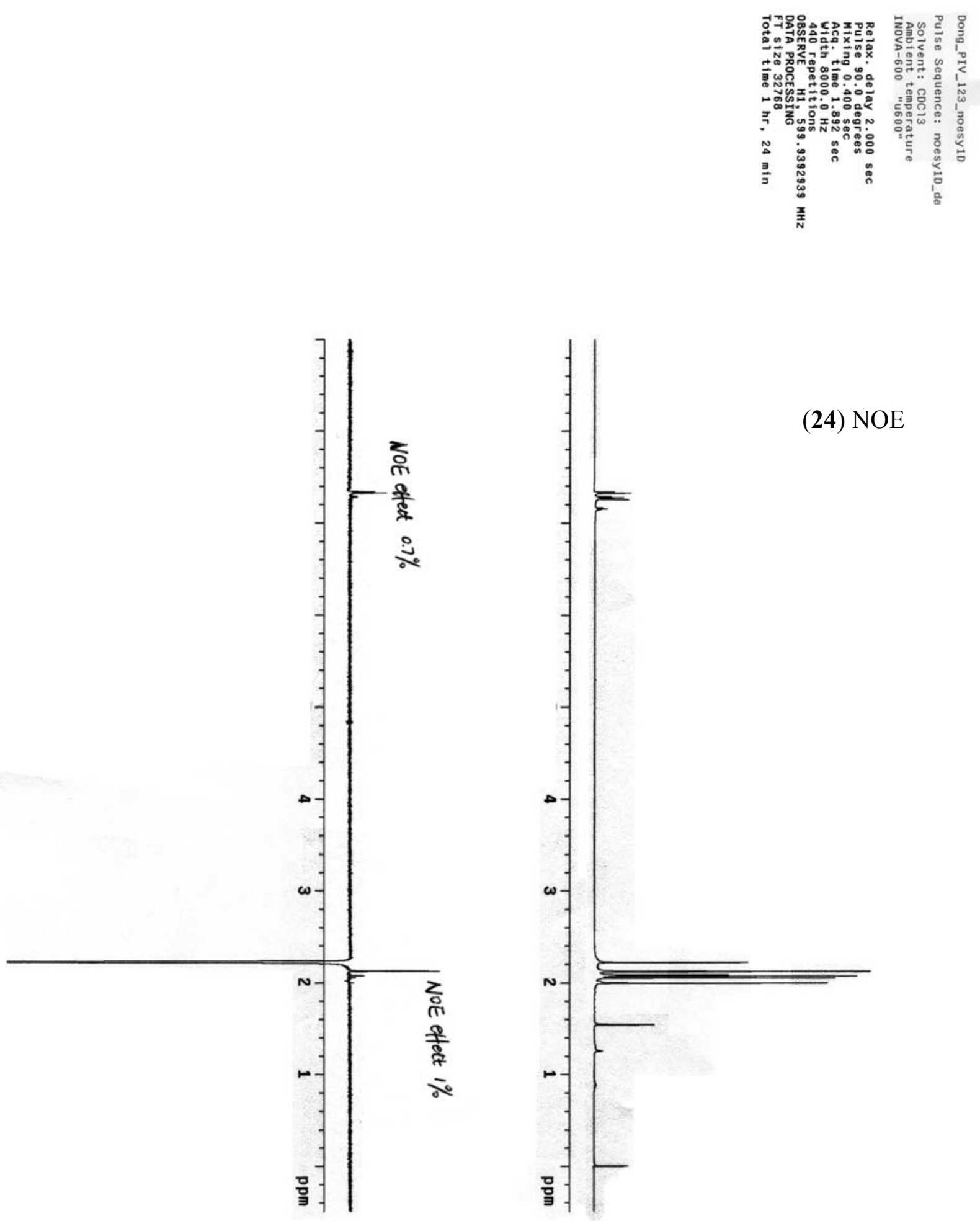

S- 60 